\title{
Elevated Temperature Tensile Tests on DU10Mo Rolled Foils TEV-2194 R1
}

\author{
Jason L. Schulthess
}

May 2018

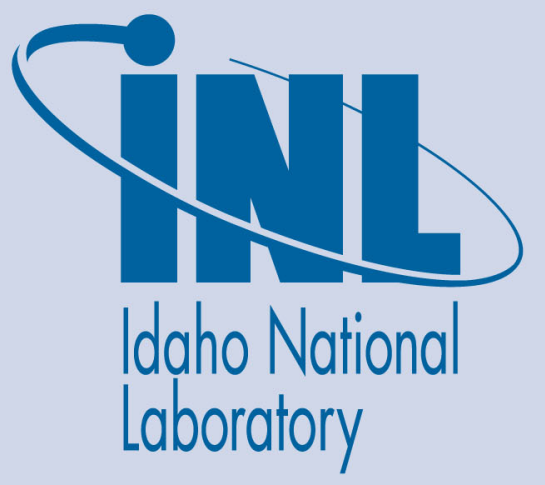

The INL is a U.S. Department of Energy National Laboratory operated by Battelle Energy Alliance 


\title{
Elevated Temperature Tensile Tests on DU10Mo Rolled Foils TEV-2194 R1
}

\author{
Jason L. Schulthess
}

May 2018

\section{Idaho National Laboratory Idaho Falls, Idaho 83415}

http://www.inl.gov

Prepared for the U.S. Department of Energy National Nuclear Security Administration Under DOE Idaho Operations Office Contract DE-AC07-05ID14517 
Rev. 03

Title: $\quad$ Elevated Temperature Tensile Tests on DU-10Mo Rolled Foils

$\begin{array}{llllll}\text { TEV No.: } & 2194 & \text { Rev. No.: } 2 & \text { Project No.: } 25228 & \text { Date: } 05 / 02 / 18\end{array}$

\begin{tabular}{|c|c|c|}
\hline 1. Quality Level (QL) No. & MFC-000936 & \multirow{6}{*}{ Professional Engineer's Stamp } \\
\hline 2. QL Determination No. & MFC-000936 & \\
\hline 3. Engineering Job (EJ) No. & $x$ & \\
\hline 4. SSC ID & $x$ & \\
\hline 5. Building & FASB & \\
\hline 6. Site Area & MFC & \\
\hline
\end{tabular}

7. Introduction:

Tensile mechanical properties for uranium-10 wt.\% molybdenum (U-10Mo) foils are required to support modeling and qualification of new monolithic fuel plate designs. It is expected that depleted uranium-10 wt\% Mo (DU-10Mo) mechanical behavior is representative of the low enriched $U-10$ Mo to be used in the actual fuel plates, therefore DU-10Mo was studied to simplify material processing, handling, and testing requirements. In this report, tensile testing of DU$10 \mathrm{Mo}$ fuel foils prepared using four different thermomechanical processing treatments were conducted to assess the impact of foil fabrication history on resultant tensile properties.

The Y-12 National Security Complex at Oak Ridge National Laboratory (Y12) provided Idaho National Laboratory (INL) with a cast and machined $\sim 4.1 \mathrm{~mm}$-thick, $\sim 127 \mathrm{~mm}$-wide, and $\sim 182$ $\mathrm{mm}$-long plate of DU-10Mo. After receipt at INL, the plate (designated alloy 551) was sectioned into four pieces using wire electric discharge machining (wire EDM), and each piece was homogenized at $1000^{\circ} \mathrm{C}$ for two hours under vacuum of $5 \times 10-6$ Torr. These pieces were then individually hot-rolled into foil strips of varying thickness. Additional cold rolling was performed on three of the four foils to bring the final thickness of each cold rolled foil to a target of $0.38 \mathrm{~mm}$, representative of the final thickness of actual fuel foils. The four foil conditions studied were as follows: Foil 551-2 - additional 50\% cold-rolling reduction; Foil 551-3 - additional 50\% coldrolling reduction followed by stress-relief annealing at $650^{\circ} \mathrm{C}$ for one hour; Foil $551-4$ - additional $20 \%$ cold-rolling reduction; and Foil 551-5 - no further processing, i.e. hot-rolled only. Due to breach of the protective rolling can, hot rolling of foil 551-5 was terminated early, resulting in a final nominal thickness of $0.53 \mathrm{~mm}$. Sub-size flat sheet-type tensile specimens, with dimensions scaled to $50 \%$ (i.e. $50 \mathrm{~mm}$ nominal overall length) of the normal subsize specimen, as specified in Figure 1 of ASTM E8/8M-13, were cut from the finished foil sheets using wire EDM machining. Tests were conducted on specimens with both longitudinal and transverse orientation relative to rolling direction, and at various temperatures between room temperature (per ASTM E8/8M-13) and elevated temperatures (per ASTM E21-10) up to $550^{\circ} \mathrm{C}$.

This work was conducted under PLN-4560, "GTRI Mechanical Property Characterization Test Plan," which contains additional requirements and instructions for testing. ${ }^{1}$ The following report presents details of the testing system, testing methods, and mechanical properties determined from the test data.

8. If revision, please state the reason and list sections and/or pages being affected: Revised to correct typographical erros in Table 1, Fig.11, Fig.12, Fig.13, Fig.15, Fig.16 
Rev. 03

Title: $\quad$ Elevated Temperature Tensile Tests on DU-10Mo Rolled Foils

$\begin{array}{lllll}\text { TEV No.: } & 2194 & \text { Rev. No.: } 2 & \text { Project No.: } 25228 & \text { Date: } 05 / 02 / 18\end{array}$

9. Conclusions/Recommendations:

Tensile properties of DU-10Mo at room temperature through approximately $400^{\circ} \mathrm{C}$ determined from the tests conducted herein suggest the material is stronger and has lower ductility than what has been reported previously in the literature. The explanation for these differences has yet to be determined, but is likely related to differences in grain size and/or impurity content. At the highest temperatures tested $\left(550^{\circ} \mathrm{C}\right)$ better agreement between the values reported here and available literature was found. As expected, yield and ultimate tensile strength decreased with increasing test temperature. Generally, the yield stress for all foil processing conditions was found to be in the range of $1100 \mathrm{MPa}$ for room temperature tests, and in the range of $200 \mathrm{MPa}$ for tests conducted at $550^{\circ} \mathrm{C}$. Ultimate tensile stress was in the range of $1175 \mathrm{MPa}$ at room temperature, decreasing to approximately $225 \mathrm{MPa}$ at $550^{\circ} \mathrm{C}$. Elongation increased significantly from $0-2 \%$ at room temperature, to $50 \%$ or more for the tests at $550^{\circ} \mathrm{C}$. Additional details on the observed effects of foil processing condition and specimen orientation on tensile properties are summarized below:

\section{Yield Strength}

No significant effect of fabrication history on yield stress was observed at the lowest temperature (room temperature) and highest temperature $\left(550^{\circ} \mathrm{C}\right)$ tested. However, tests indicated yield strength differences exist at the intermediate temperatures tested with the $50 \%$ cold worked and annealed, and hot rolled only material producing lower yield stress at the intermediate test temperatures. Significant effects of specimen orientation on yield strength were only observed in a few cases (specifically $20 \%$ cold worked material tested at $200^{\circ} \mathrm{C}$ resulted in lower yield stress in the transvers orientation, and hot-rolled only material tested at room temperature and $200^{\circ} \mathrm{C}$ where the transvers orientation resulted in slightly higher yield stress).

\section{Ultimate Tensile Strength (UTS)}

Significant differences in the UTS were noted in the longitudinal direction for the four foils at room temperature, with the difference continuing, but converging as the temperature increased. This difference between each of the four foils was also found to exist in the transvers direction. The $50 \%$ cold worked and annealed, and hot rolled only material produced lower ultimate tensile stress at room and the intermediate test temperatures, but converged when tested at $550^{\circ} \mathrm{C}$.

Orientation effects for specimens from the same foil were only noted in the following case: $50 \%$ cold worked at $350^{\circ} \mathrm{C}$ in which the transvers direction produced lower ultimate tensile stress. All other tests indicated no significant anisotropy due to rolling direction.

\section{Slope}

Slope of the initial section of the stress-strain curve was calculated for each specimen and then averaged across all specimens tested in each temperature group. The average slope in $\mathrm{MPa} / \%$ and standard deviation for each test temperature are: $20^{\circ} \mathrm{C}: 884.21 \pm 13.15 ; 200-250^{\circ} \mathrm{C}$ : $417.63 \pm 16.81$; $350^{\circ} \mathrm{C}: 422.25 \pm 14.75 ; 400-450^{\circ} \mathrm{C}: 320.43 \pm 17.88 ; 550^{\circ} \mathrm{C}: 199.29 \pm 24.41$. Room-temperature elastic-modulus values reported in the literature are in the low-to-mid-80s GPa range ${ }^{2,3}$ _ consistent with our room-temperature test results. 
Rev. 03

Title: $\quad$ Elevated Temperature Tensile Tests on DU-10Mo Rolled Foils

$\begin{array}{lllll}\text { TEV No.: } 2194 & \text { Rev. No.: } 2 & \text { Project No.: } 25228 & \text { Date: } 05 / 02 / 18\end{array}$

\section{Ductility}

Room temperature ductility was determined for all of the foil conditions tested. Of note, the $50 \%$ cold worked and annealed foil (foil 551-3) showed increased ductility compared to the $50 \%$ and $20 \%$ cold worked foils. The hot rolled only foil showed more ductility in the longitudinal direction than any other foil at room temperature, but showed very little ductility in the transverse direction, indicating significant anisotropy at room temperature.

Ductility increased continuously for all foil conditions as testing temperature increased, and was significant for foils tested at $550^{\circ} \mathrm{C}$. At $550^{\circ} \mathrm{C}$, the ductility for all foil conditions and orientations increased from $\sim<10 \%$ to as much as $\sim 70 \%$ in the $50 \%$ cold worked foil in the longitudinal direction (551-2) and also $\sim 70 \%$ for the $50 \%$ cold worked and annealed foil (551-3) in both directions. The increase in ductility at $550^{\circ} \mathrm{C}$ was more pronounced for the $20 \%$ cold worked foil than for other foil processing conditions.

For a few specimens in both room temperature and elevated temperature cases, the $0.2 \%$ offset curve did not intersect the stress strain curve. Theoretically, ductility would be very small in these cases. When combined with the measurement error evaluated in Table 5, and recognizing that it can be difficult to accurately piece specimens back together for post-test elongation measurements due to roughness at the fracture surface; it is likely that actual elongation values reported are less than the reported values of $3 \%$ or less. This specifically includes the following specimens tested at room temperature: 551-2-2 L17, and T14; 551-5 T1, and T2; and the following specimens tested at elevated temperature: 551-2-2 T11, and T5, but may impact any specimens with reported elongation values of less than $3 \%$.

\section{Recommendations for Future Work}

It is recommended that future work include fractography of selected specimens to determine if failure mechanisms other than ductile rupture exist, and whether fracture initiation sites can be identified. Metallography and microstructural characterization should be completed to characterize grain sizes and other microstructural features that may explain the observed mechanical behavior. Finally, it is recommended that additional testing be conducted on similarly processed material having different impurity content (particularly different carbide distributions) to better understand the range of properties that may be expected in commercially fabricated fuel foils. 
TEM-10300-1

$03 / 01 / 2012$

TECHNICAL EVALUATION

Page 4 of 38

Rev. 03

Title: $\quad$ Elevated Temperature Tensile Tests on DU-10Mo Rolled Foils

$\begin{array}{lllll}\text { TEV No.: } 2194 & \text { Rev. No.: } 2 & \text { Project No.: } 25228 & \text { Date: } 05 / 02 / 18\end{array}$

\section{CONTENTS}

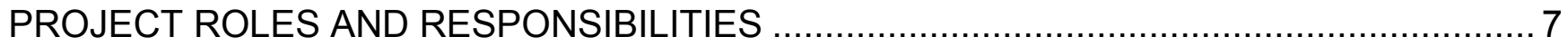

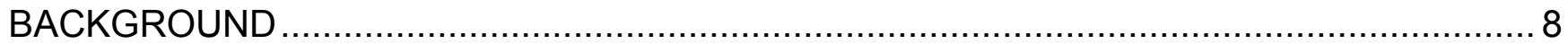

TEST SPECIMEN AND TEST SYSTEM PREPARATION $\ldots \ldots \ldots \ldots \ldots \ldots \ldots \ldots \ldots \ldots \ldots \ldots \ldots \ldots . . \ldots$

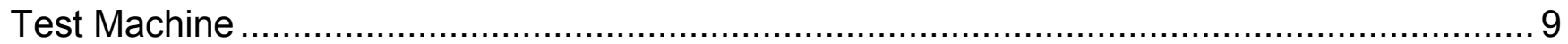

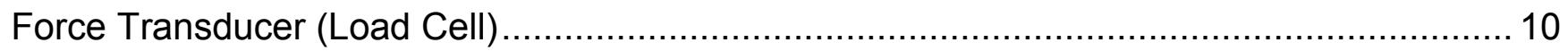

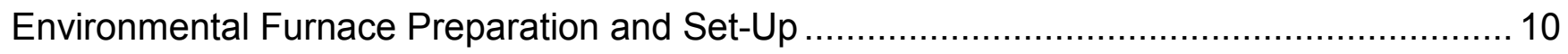

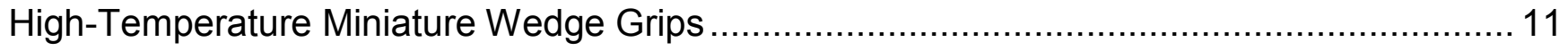

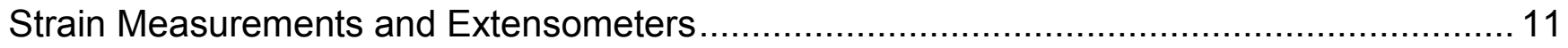

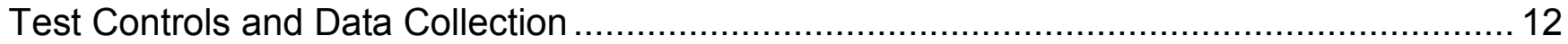

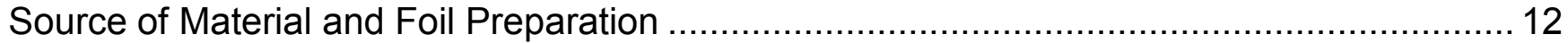

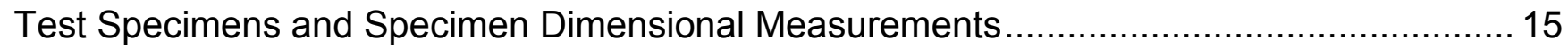

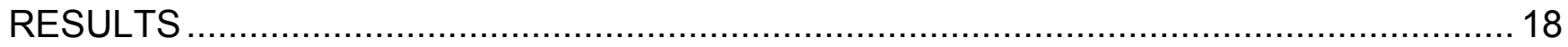

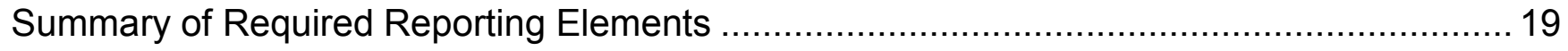

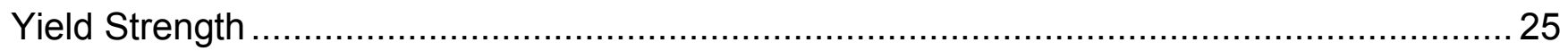

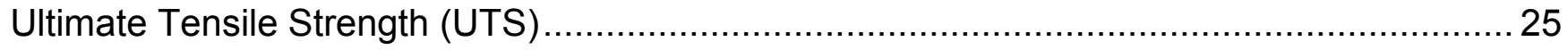

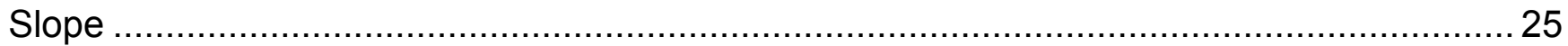

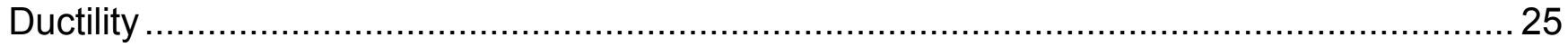

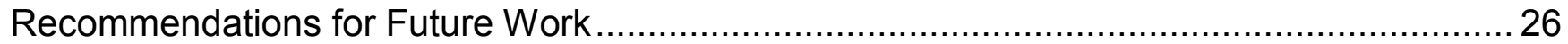

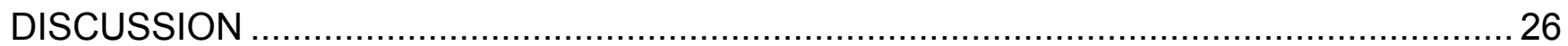

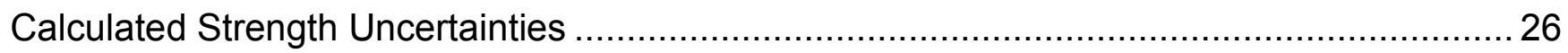

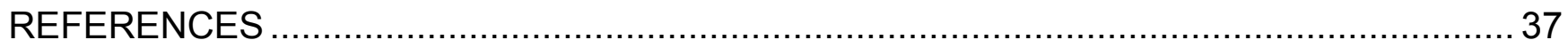


Rev. 03

Title:

Elevated Temperature Tensile Tests on DU-10Mo Rolled Foils

TEV No.: 2194

Rev. No.: 2

Project No.:

25228

Date: $05 / 02 / 18$

\section{FIGURES}

Figure 1. Half-subsize flat tensile specimen fabrication specification (INL Drawing 604240). ${ }^{12}$

Figure 2. Machined coupon from $\mathrm{Y}-12$ quartered into sections for rolling. Figure also shows scrap containing a visible casting defect that was cut off during sectioning.

Figure 3. Foil 551-4-1 after hot rolling and cold rolling has been completed.

Figure 4. EDM pattern showing the cutting diagram for Foil 551-2-2.

Figure 5. Foil 551-2-2 showing subsection labeling corresponding to the EDM cutting pattern.

Figure 6. Individually labeled tensile specimens as cut per EDM pattern for Foil 551-2-2.

Figure 7. Gauge-marking specimen-support base.

Figure 8. Marking indenter guide plate installed over specimen on support base plate. Punch is placed in one of the guide holes. Finger pressure produces a satisfactory gauge-mark indent.

Figure 9. Stainless steel trial specimen showing gauge-mark indents produced using the marking fixture. The reduced section width is approximately $3.0 \mathrm{~mm}$. The marks shown appear larger than they actually are due to high contrast and lighting angle.

Figure 10. Stress-strain plots for room-temperature and $250^{\circ} \mathrm{C}$ tensile tests on longitudinal specimens of DU-10Mo foil 551-2 that was prepared by hot-rolling, followed by $50 \%$ cold-rolling reduction in thickness. Slope of offset modulus lines (dashed) represents the modulus reduction expected between room temperature and $250^{\circ} \mathrm{C}$.

Figure 11. Yield stress in the longitudinal direction for each of the four foil fabrication conditions. The figure shows the cluster of yield stress at room temperature and at $550^{\circ} \mathrm{C}$ while there is a divergence in the fabrication conditions in the intermediate values. The annealed and hot-rolled-only foils indicate lower yield stress values in the intermediate temperatures.

Figure 12. Ultimate tensile stress in the longitudinal direction for each of the four foil-fabrication conditions. The figure shows the cluster of ultimate tensile stress at $550^{\circ} \mathrm{C}$ while there is a divergence in the fabrication conditions in the room temperature and intermediate values. The annealed and hot-rolled-only foils indicate lower ultimate tensile stress values in the room and intermediate temperatures.

Figure 13. Yield stress in the transverse direction for each of the four foil-fabrication conditions. The figure shows the cluster of yield stress at room temperature and at $550^{\circ} \mathrm{C}$ while there is a divergence in the fabrication conditions in the intermediate values. The annealed and hot-rolled only foils indicate lower yield stress values in the intermediate temperatures. Note that data are not available for foils 551-4 and 551-5 in the transverse direction at $400^{\circ} \mathrm{C}$.

Figure 14. Ultimate tensile stress in the transverse direction for each of the four foil-fabrication conditions. The figure shows the cluster of yield stress at room temperature and at $550^{\circ} \mathrm{C}$ while there is a divergence in the fabrication conditions in the intermediate values. The annealed and hot-rolled-only foils indicate lower yield stress values in the intermediate temperatures $\left(200^{\circ} \mathrm{C}\right)$, but this seems to reconverge at $400^{\circ} \mathrm{C}$. Note that data are not available for foils 551-4 and 551-5 in the transverse direction at $400^{\circ} \mathrm{C}$. 
Rev. 03

Title: $\quad$ Elevated Temperature Tensile Tests on DU-10Mo Rolled Foils

$\begin{array}{lllll}\text { TEV No.: } & 2194 & \text { Rev. No.: } 2 & \text { Project No.: } 25228 & \text { Date: } 05 / 02 / 18\end{array}$

Figure 15. Yield stress for foils cold-rolled to $50 \%$ reduction. Note no significant difference in yield stress values based on rolling direction. No data are available for yield stress in the transvers direction for 350 and $450^{\circ} \mathrm{C}$.

Figure 16. Ultimate tensile stress for foils cold-rolled to $50 \%$ reduction and then annealed at $650^{\circ} \mathrm{C}$ for one hour. Note no significant difference in ultimate tensile stress values based on rolling direction.

Figure 17. Yield stress for foils cold-rolled to $50 \%$ reduction and then annealed at $650^{\circ} \mathrm{C}$ for one hour. Note no significant difference in yield stress values based on rolling direction.

Figure 18. Ultimate tensile stress for foils cold-rolled to $50 \%$ reduction and then annealed at $650^{\circ} \mathrm{C}$ for one hour. Note no significant difference in ultimate tensile stress values based on rolling direction.

Figure 19. Yield stress for foils cold-rolled to $20 \%$ reduction. Note a minor difference in yield stress values based on rolling direction for $200^{\circ} \mathrm{C}$. No transverse data are available for $400^{\circ} \mathrm{C}$.

Figure 20. Ultimate tensile stress for foils hot-rolled only with no cold-rolling reduction. Note no significant difference in ultimate tensile-stress-values-based temperature. No transverse data are available for $400^{\circ} \mathrm{C}$.

Figure 21. Yield stress for foils hot-rolled only with no cold-rolling reduction. Note no significant difference in yield stress values based temperature. No transverse data available for $400^{\circ} \mathrm{C}$

Figure 22. Ultimate tensile stress for foils hot-rolled only with no cold-rolling reduction. Note no significant difference in ultimate tensile stress values based temperature. No transverse data available for $400^{\circ} \mathrm{C}$.

Figure 23. Minimal area inside furnace to install grip alignment collar, install specimen in grips, ensure proper specimen alignment in grips, tighten grip jaws, remove alignment collar, and install extensometer (when used). Care must be used to prevent damage to specimens or fragile Super Kanthal heating elements.

Figure 24. Inert-gas and cooling-water plumbing at rear of furnace. Metal vacuum hose running down to pump is seen at lower right. Gas piping is all metal or ceramic to prevent oxygen infusion.

Figure 25. Specimen installed in grips inside of furnace chamber, with extensometer installed, ready to begin a room-temperature test. Wires from thermocouples welded to grip jaws are seen above and below extensometer. For size reference, the hex grip-tightening bolt-heads are $16 \mathrm{~mm}$. Grip alignment collar has been removed.

Figure 26. Broken elevated-temperature test specimen as seen through magnifying viewport in furnace door. Lighting is through a narrow window in the door below the viewport. Break is visible at lower portion of reduced section. 
TEM-10300-1

$03 / 01 / 2012$

TECHNICAL EVALUATION

Page 7 of 38

Rev. 03

Title: $\quad$ Elevated Temperature Tensile Tests on DU-10Mo Rolled Foils

$\begin{array}{lllll}\text { TEV No.: } 2194 & \text { Rev. No.: } 2 & \text { Project No.: } 25228 & \text { Date: } 05 / 02 / 18\end{array}$

PROJECT ROLES AND RESPONSIBILITIES

\begin{tabular}{l|l|l|l}
\multicolumn{1}{c|}{ Project Role } & Name (Typed) & Organization & Pages covered (if applicable) \\
\hline Performer & Jason Schulthess & U510 & See eCR 659331 \\
Performer & Micheal Heighes & B120 & See eCR 659331 \\
Checker $^{\mathrm{a}}$ & Barry H. Rabin & C620 & See eCR 659331 \\
Independent Reviewer $^{\mathrm{b}}$ & Wilson R. Lloyd & B120 & See eCR 659331 \\
CUl Reviewer & N/A & N/A & N/A \\
Manager & Mitch Meyer & C600 & See eCR 659331 \\
Requestor $^{\mathrm{e}}$ & Jim Cole & C610 & See eCR 659331 \\
Nuclear Safety & N/A & N/A & N/A \\
Document Owner & Jim Cole & C610 & See eCR 659331 \\
Responsibilities & & & \\
\hline
\end{tabular}
a. Confirmation of completeness, mathematical accuracy, and correctness of data and appropriateness of assumptions.
b. Concurrence of method or approach. See definition, LWP-10106.
c. Concurrence with the document's markings in accordance with LWP-11202.
d. Concurrence of procedure compliance. Concurrence with method/approach and conclusion.
e. Concurrence with the document's assumptions and input information. See definition of Acceptance, LWP-10200. 
TEM-10300-1

$03 / 01 / 2012$

TECHNICAL EVALUATION

Page 8 of 38

Rev. 03

Title: $\quad$ Elevated Temperature Tensile Tests on DU-10Mo Rolled Foils

$\begin{array}{llllll}\text { TEV No.: } 2194 & \text { Rev. No.: } 2 & \text { Project No.: } 25228 & \text { Date: } 05 / 02 / 18\end{array}$

\section{BACKGROUND}

Tensile properties for rolled uranimum-10 wt.\% molybdenum ( $U-10 \mathrm{Mo})$ foils are required to support modeling and qualification of new monolithic fuel plate designs that incorporate these alloy foils as fuel. Limited data exist on the property-processing-structure relationship of U-10Mo fuel foils. Most of the available studies reporting properties for U-Mo alloys were conducted in the 1950s and 1960s. For example, Waldron (1958) reports yield stress, ultimate tensile stress, and modulus for U-Mo alloys, where the $w t \%$ of Mo was varied in the alloy and where the heat treatment temperature and time were varied (see Table 1). ${ }^{4}$ However, the Waldron report does not provide information relevant to the properties of rolled foils, or the effect of foil rolling conditions on properties.

Table 1. Summarized mechanical properties from Waldron. ${ }^{4}$

\begin{tabular}{|c|c|c|c|c|c|c|c|c|}
\hline 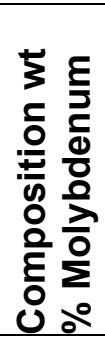 & 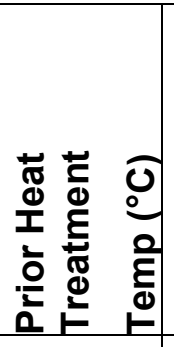 & 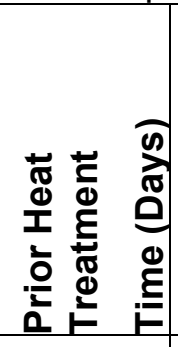 & 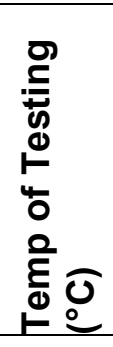 & 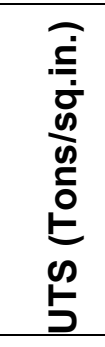 & $\begin{array}{l}\frac{\sigma}{\pi} \\
\sum^{\frac{\sigma}{\Sigma}} \\
0 \\
5 \\
5\end{array}$ & 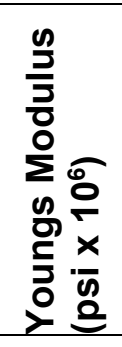 & 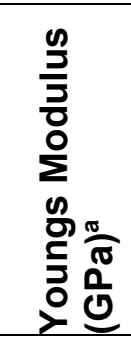 & 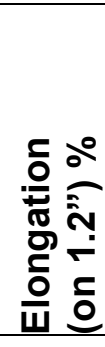 \\
\hline 10 & 900 & 7 & 20 & 44.8 & 617.8 & 12.6 & 86.87 & 0.1 \\
\hline 10 & 450 & 14 & 20 & 21.3 & 293.7 & 17.3 & 119.3 & 0.8 \\
\hline 10 & 900 & 7 & 200 & 37 & 510.2 & 10.7 & 73.77 & 0.5 \\
\hline 10 & & & 400 & 26 & 358.5 & 7.5 & 51.71 & 1 \\
\hline 10 & & & 600 & 13 & 179.3 & 4.8 & 33.09 & 0 \\
\hline 10 & & & 800 & 4 & 55.16 & 6.0 & 41.37 & 3.0 \\
\hline 10 & 450 & 14 & 200 & 22 & 303.4 & 13.3 & 91.7 & Nil \\
\hline 10 & & & 300 & 13.3 & 183.4 & 15.0 & 103.4 & 0.5 \\
\hline 10 & & & 400 & 18.6 & 256.5 & 15.8 & 108.9 & 0.5 \\
\hline 10 & 575 & 28 & 400 & 10.8 & 148.9 & 12.2 & 84.12 & 2 \\
\hline 10 & & & 600 & 9 & 124.1 & 8.6 & 59.29 & 0.5 \\
\hline 10 & & & 800 & 6.3 & 86.9 & 8.6 & 59.29 & 11 \\
\hline
\end{tabular}

Other previous work by various authors to establish mechanical properties of $U-10 \mathrm{Mo}$ alloy is summarized by Ozaltun et al. in Table $2 .{ }^{5}$ Burkes et al (2009) also summarized previous work reporting mechanical properties of U-Mo alloys. These results are reported based on variation of Mo content in the alloy. Yield stress, ultimate tensile stress, and elongation, all increase with increasing Mo content and is attributed to the improved resistance to bulk plastic deformation through increased addition of Mo and associated hardening effect in the $y$ phase. Burkes et all (2009) compare their results to previous available literature, but note that differences in homogenization treatment, specimen geometry and strain rates makes direct comparison difficult. ${ }^{6}$ 
Rev. 03

Title: $\quad$ Elevated Temperature Tensile Tests on DU-10Mo Rolled Foils

$\begin{array}{lllll}\text { TEV No.: } & 2194 & \text { Rev. No.: } 2 & \text { Project No.: } 25228 & \text { Date: } 05 / 02 / 18\end{array}$

The previously reported properties (summarized in both [5], and [6]) are only valid for the specific thermo-mechanical treatments considered, and testing was performed at a limited selection of temperatures. Fabrication history and thermal treatment of the alloy can have a significant impact on the resultant mechanical properties. Thus, an expanded set of mechanical properties for various foil rolling conditions typical of fuel foil manufacturing is necessary. This work aims to evaluate the mechanical properties of U-10Mo alloys under various representative rolling conditions to inform modeling efforts, fabrication process development, and ultimately, to support fuel qualification and reactor conversion.

Table 2. U-10Mo properties summarized by Ozaltun. ${ }^{5}$

\begin{tabular}{|c|c|c|c|c|c|c|c|}
\hline \multicolumn{7}{|c|}{ Fuel Foil (U-10Mo) } \\
\hline Young's Modulus & \multicolumn{2}{c|}{ Poisson's ratio } & \multicolumn{2}{c|}{ Density } & \multicolumn{2}{c|}{ Yield Stress } \\
\hline${ }^{\circ} \mathrm{C}$ & $\mathrm{GPa}$ & ${ }^{\circ} \mathrm{C}$ & $(-)$ & ${ }^{\circ} \mathrm{C}$ & $\left(\mathrm{kg} / \mathrm{m}^{3}\right)$ & ${ }^{\circ} \mathrm{C}$ & $\mathrm{MPa}$ \\
\hline 21 & 65.00 & 25 & 0.35 & 21 & 16,750 & 21 & 780 \\
\hline & & & & 100 & 16,380 & 94 & 760 \\
\hline & & & & 200 & 16,310 & 205 & 655 \\
\hline & & & & 300 & 16,230 & 316 & 527 \\
\hline & & & & 400 & 16,140 & 427 & 474 \\
\hline & & & & 500 & 16,060 & 539 & 427 \\
\hline & & & & 600 & 15,980 & & \\
\hline
\end{tabular}

\section{TEST SPECIMEN AND TEST SYSTEM PREPARATION}

\section{Test Machine}

The testing system incorporates a standard Instron 3366 table-top test machine, Instron $5 \mathrm{kN}$ load cell, Instron high-temperature wedge grips, and a C-M 1600-series environmental furnace. The system was developed, calibrated, and verified for performing this series of tests. The system includes an environmental-control furnace that allows elevated-temperature tensile testing in an inert-gas environment with low oxygen concentration, preventing rapid oxidation or oxygen embrittlement of the DU-10Mo test specimens. Room temperature tests on some of these specimens were performed in air to establish baseline properties for this particular material condition. A strain-estimating algorithm was developed using the room-temperature data to establish correlation between machine crosshead displacement and specimen reduced section strain, allowing elevated-temperature specimen strain to be estimated from test-machine crosshead displacement data. Other factors unique to the elevated-temperature test configuration were also assessed, and methods to account for factors that are not directly measurable in these tests were developed. Further details of the test-system configuration and performance-verification processes are detailed below and documented in [7]. 
Rev. 03

Title: $\quad$ Elevated Temperature Tensile Tests on DU-10Mo Rolled Foils

$\begin{array}{llllll}\text { TEV No.: } & 2194 & \text { Rev. No.: } 2 & \text { Project No.: } 25228 & \text { Date: } 05 / 02 / 18\end{array}$

\section{Force Transducer (Load Cell)}

Load-cell accuracy was verified by the INL Standards and Calibration Laboratory (S\&CL) using established procedures. In accordance with ASTM E4-10, the maximum allowable load-cell error is the greater of $\pm 0.50 \%$ of any force reading (proportional error), or $\pm 0.25 \%$ of load-cell full-scale capacity (fixed error). The load cell was within its calibration time interval for all tests performed. The data sheet from the most recent calibration is replicated as Table 3.

Table 3. Load cell calibration record data (12 Nov 2013, INL S\&CL).

\begin{tabular}{|l|l|l|l|}
\hline $\begin{array}{c}\text { Instron Reading (N) } \\
\text { (Accuracy +/-1 digit) }\end{array}$ & $\begin{array}{l}\text { Reference } \\
\text { Reading (N) }\end{array}$ & $\begin{array}{l}\text { Indicated } \\
\text { Error (N) }\end{array}$ & $\begin{array}{c}\text { Error (\% of } \\
\text { Instron Reading) }\end{array}$ \\
\hline $\mathbf{0 . 0}$ & $\mathbf{0 . 0}$ & $\mathbf{0 . 0}$ & NA \\
\hline $\mathbf{1 0 3 2 \pm 1}$ & $\mathbf{1 0 3 4 . 4 8}$ & $\mathbf{- 2 . 4 8}$ & $\mathbf{- 0 . 1 4}$ to $-\mathbf{0 . 3 4}$ \\
\hline $1873 \pm 1$ & 1877.49 & -4.49 & -0.19 to -0.29 \\
\hline $3262 \pm 1$ & 3267.47 & -5.47 & -0.14 to -0.20 \\
\hline $4232 \pm 1$ & 4237.43 & -5.43 & -0.10 to -0.15 \\
\hline $\mathbf{0 . 0}$ & $\mathbf{0 . 0}$ & $\mathbf{0 . 0}$ & NA \\
\hline $\mathbf{9 8 8 . 6 \pm 0 . 1}$ & $\mathbf{9 9 1 . 4 8}$ & $\mathbf{- 2 . 8 8}$ & $-\mathbf{0 . 2 8}$ to -0.29 \\
\hline $2286 \pm 1$ & 2291.53 & -5.53 & -0.20 to -0.29 \\
\hline $3231 \pm 1$ & 3236.98 & -5.98 & -0.15 to -0.22 \\
\hline $4208 \pm 1$ & 4214.14 & -6.14 & -0.12 to -0.22 \\
\hline
\end{tabular}

The potential for force indication error adds to the uncertainty of the calculated stress values. For the majority of tests, the ASTM allowable error limit of $0.25 \%$ of full scale capacity is the relevant limiting value, and corresponds to $\pm 12.5 \mathrm{~N}$. For a nominal specimen cross-section of $1.2 \mathrm{~mm}^{2}$, this is a maximum potential stress error of $\pm 10 \mathrm{MPa}$. However, typical errors within the range of yield strength and UTS measurements during these tests $(1000 \mathrm{~N})$ were consistently about $0.30 \%$ greater than the machine reading (data obtained from the load-cell calibration data sheets on file at S\&CL). This correlates to errors nominally proportional to the indicated values, and smaller than $3 \mathrm{~N}$ in the range of interest. For practical purposes, though not assured, the actual applied force appears consistently $0.3 \%$ higher than the machine indication. The specimen initial-area error due to initial width and thickness measurement uncertainty is so small $(<0.01 \%$ of cross-section error) that it is inconsequential.

\section{Environmental Furnace Preparation and Set-Up}

The furnace's inert-gas plumbing was modified to include an electrical feed-through to accommodate additional thermocouple leads for specimen- and grip-temperature monitoring. Thermocouples were welded to the exposed end of the upper and lower grip jaws immediately adjacent to the jaw-to-specimen contact region. Calibration testing with instrumented surrogate test specimens showed that test-specimen temperature was accurately reflected by the instrumented grip-jaw temperature as recorded in [8]. Based on these tests, test-specimen temperature during all elevated-temperature testing was inferred from thermally equilibrated grip jaw temperatures, where both grips were holding constant over time and within $5^{\circ} \mathrm{C}$ of the target test temperature. 
Rev. 03

Title: $\quad$ Elevated Temperature Tensile Tests on DU-10Mo Rolled Foils

$\begin{array}{lllll}\text { TEV No.: } 2194 & \text { Rev. No.: } 2 & \text { Project No.: } 25228 & \text { Date: } 05 / 02 / 18\end{array}$

A vacuum pump and appropriate isolation valves were also connected to the inert-gas piping system. Cycles of evacuation (with a roughing-type vacuum pump) and argon backfill/purge were completed prior to heating to reduce the oxygen concentration below $20 \mathrm{ppm}$, as indicated on an $\mathrm{O}_{2}$ concentration meter attached to the furnace-outlet gas piping. Typical $\mathrm{O}_{2}$ concentration values were less than $5 \mathrm{ppm}$ after 4 vacuum/purge cycles. Approximately halfway through the testing, it was determined that the same $\mathrm{O}_{2}$-concentration values could be obtained after a single cycle of vacuum and argon-gas purge. Argon-gas flow rate through the furnace during heating and testing was $5 \mathrm{l} / \mathrm{min}$ (nominal) at a maximum pressure of $10 \mathrm{kPa}$ (gauge pressure inside furnace chamber).

\section{High-Temperature Miniature Wedge Grips}

The original jaw inserts provided by Instron Corp. were made from a soft nickel alloy and were intended for use with pin-loaded specimen-end tabs. The serrations of these jaws were too soft to adequately grip the test specimens. Custom grip jaws were fabricated at INL for the Instron-grip bodies to accommodate the tensile specimen-end tabs used in these tests. These replacement jaws were fabricated from $\mathrm{H}-13$ tool steel, and the specimen contact faces received Surfalloy friction coatings to reliably grip the specimen end tabs over the full range of temperatures and forces that would be used. Approximately halfway through the testing program, the original Instron grip-tightening mechanism failed in one grip. INL designed and fabricated a new mechanism and replaced the mechanisms on both grip bodies. These new mechanisms performed well throughout the remainder of the testing. They facilitated easier specimen installation and removal from the grips as well. The grip-tightening mechanism failure and replacement with new parts did not influence any of the test results.

An alignment collar was fabricated that holds the grip bodies in alignment while the specimen is installed and the grip jaws, tightened. The design and use of this alignment collar are discussed in [7]

\section{Strain Measurements and Extensometers}

Room-temperature testing was completed using a small $12.7 \mathrm{~mm}$ gauge-length extensometer from Epsilon Corp. The extensometer has a measuring range to $+20 \%$ tensile strain. At intervals not exceeding 24 hours prior to beginning any test, the extensometer was recalibrated (as necessary), and accuracy was verified in accordance with requirements of ASTM E83-10. ${ }^{9}$ The extensometer accuracy meets requirements for Class B-2 (the greater value of either $\pm 0.5 \%$ of any reading, or $200 \mu \varepsilon$ ) as prescribed within ASTM E8/E8M-13.

The test system and environmental-control furnace used for elevated-temperature testing precluded use of specimen-mounted extensometry for strain measurement. In lieu of direct strain measurement, the test system compliance was measured at room temperature, and a quadratic strain-estimating function was developed. Inputs include the quadratic coefficients, the instantaneous applied force, and the effective gauge length of the specimen. The estimated strain and actual stress data for each specimen were used to estimate the yield strength and uniform elongation for each test. Equation 1 and Equation 2 make the correlation used to determine approximate specimen strain from system cross head displacement 
TEM-10300-1

$03 / 01 / 2012$

TECHNICAL EVALUATION

Page 12 of 38

Rev. 03

Title: $\quad$ Elevated Temperature Tensile Tests on DU-10Mo Rolled Foils

$\begin{array}{lllll}\text { TEV No.: } 2194 & \text { Rev. No.: } 2 & \text { Project No.: } 25228 & \text { Date: } 05 / 02 / 18\end{array}$

$$
\begin{gathered}
\text { XhdCorr }=1.87111 * 10^{-8} * N^{2}+1.10009 * 10^{-4} * N \\
S S=\frac{\text { Xhd }- \text { XhdCorr }}{\text { Gleff }}
\end{gathered}
$$

where

SS = approximate specimen strain (\%)

Xhd $=$ cross-head displacement $(\mathrm{mm})$

XhdCorr $=$ correction for cross-head displacement $(\mathrm{mm})$

$\mathrm{N}=$ force $(\mathrm{N})$

GLeff $=$ effective gauge length $(\mathrm{mm} / \%)$.

The method used to estimate specimen strain from grip displacement for the elevated temperature tests works well and provided consistent results from the suite of valid elevated-temperature tests that were completed.

\section{Test Controls and Data Collection}

ASTM E8/8M-13 or ASTM E21-10 guided room-temperature and elevated-temperature testing, respectively. Room-temperature tests occurred in an air environment. Elevated-temperature tests were performed at temperatures varying between 200 and $550^{\circ} \mathrm{C}$ in an argon-gas environment.

Room-temperature and elevated-temperature tests were run at a constant crosshead speed of $0.2 \mathrm{~mm} / \mathrm{min}$, producing a nominal specimen strain rate of $0.5 \% / \mathrm{min}$. This rate meets the requirements of both ASTM E8-13 (for room temperature ${ }^{10}$ and E21-10 (for elevated-temperature ${ }^{11}$ ) tensile-testing standards. Elevated-temperature tests were conducted with the test temperature within $\pm 5^{\circ} \mathrm{C}$ of the target temperature, as measured using the calibrated thermocouples attached to the specimen ends of the grip jaws.. At the higher temperatures tested, some strain-rate effect may be present in the measured stress (see discussion section for further information).

Tests were controlled and data collected by Instron Bluehill version 3.41 software. Test method files for control and data acquisition were verified with dummy specimen tests prior to use in running actual tests on the DU-10Mo specimens. All tests were performed in accordance with PLN-4560.

\section{Source of Material and Foil Preparation}

Simulated fuel foils were fabricated using depleted uranium (DU) and molybdenum to simulate actual fuel foils made with low-enriched uranium (LEU). Flat, rectangular tensile specimens corresponding to a reduced sub-size specimen, as described in Figure 1 of ASTM E8/8M-13, ${ }^{10}$ with an additional size reduction of $50 \%$, as shown in Figure $1 .{ }^{12}$ The specimens were cut from the foil sheet using wire EDM machining. Specimens were sectioned from the sheet with the tensile axis both parallel and transverse to the foil-rolling direction. The resultant specimens have a nominal overall length of $50.8 \mathrm{~mm}$, a reduced section width of $3.2 \mathrm{~mm}$, and a reduced section length of $16 \mathrm{~mm}$. The small specimen size is necessary due to size limitations imposed by the furnace internal dimensions and material availability. 
TEM-10300-1

$03 / 01 / 2012$

TECHNICAL EVALUATION

Page 13 of 38

Rev. 03

Title: $\quad$ Elevated Temperature Tensile Tests on DU-10Mo Rolled Foils

$\begin{array}{llllll}\text { TEV No.: } & 2194 & \text { Rev. No.: } 2 & \text { Project No.: } 25228 & \text { Date: } 05 / 02 / 18\end{array}$

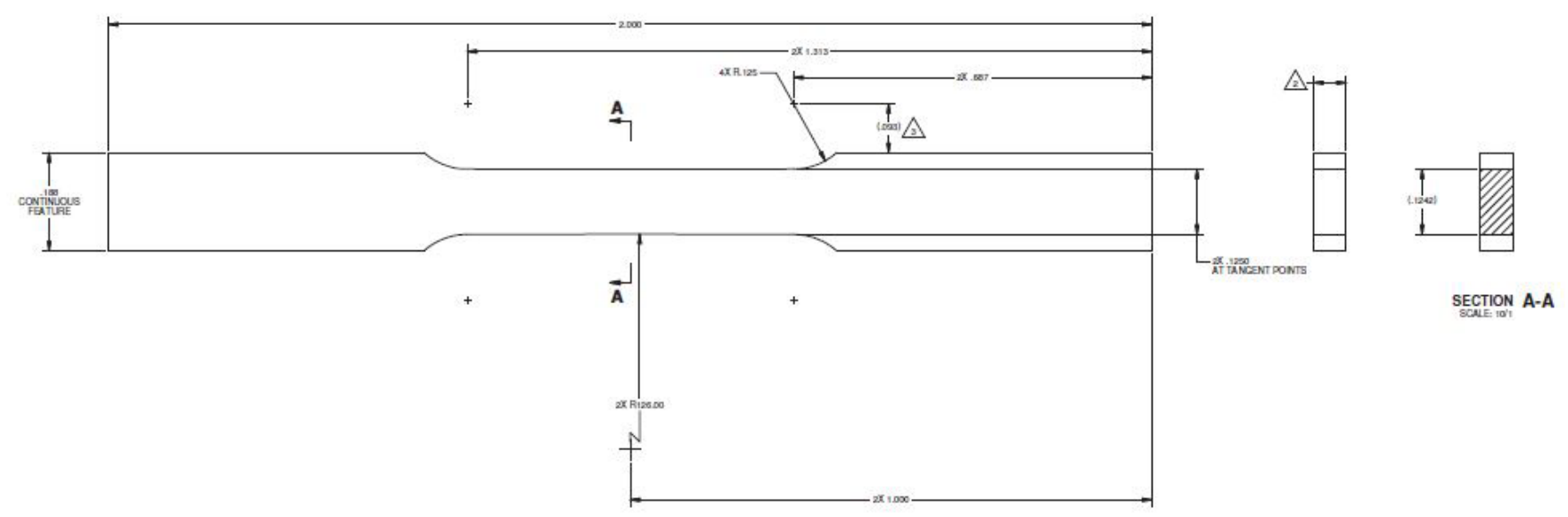

Figure 1. Half-subsize flat tensile specimen fabrication specification (INL Drawing 604240). ${ }^{12}$

The source of DU-10Mo alloy material was coupon \#551, provided by the $Y-12$ National Security Complex at Oak Ridge National Laboratory. This coupon was chosen as it was cast as part of a rolling study being conducted by the Fuel Fabrication Pillar (FFC), and thus would have similar alloy characteristics to the coupons used by FFC for the rolling studies being conducted.

Once received at Idaho National Laboratory, the coupon (plate) was quartered using wire electric discharge machining (wire EDM), as shown in Figure 2, and homogenized at $1000^{\circ} \mathrm{C}$ for two hours, while under vacuum at $5 \times 10^{-6}$ Torr.

Chemistry analysis was performed at $Y-12$ on a sister billet to the billet from which this coupon was cut and is reported for samples taken near the top of the log, near the middle, and near the bottom of the log. The Mo content is $10.4,10.5$, and $10.3 \%$ respectively. Carbon impurities are $706,714,722 \mathrm{ppm}$ respectively. Other impurities over 25 ppm are shown in Table 9 in appendix B. Impurity values less than 25 ppm where provided in the chemistry report from $\mathrm{Y}-12$ but are not reported here.

The resulting four pieces were rolled into simulated fuel foils (Figure 3 ) using general procedures described elsewhere, except with varying thermo-mechanical processing histories. ${ }^{13}$ Several material conditions of potential interest to fabricators, fuel designers, and reactor operators were created by various alterations to the rolling and heat-treating schedules. The results reported here include (1) hot-rolling followed by $50 \%$ cold-rolling (551-2), (2) hot-rolling followed by $50 \%$ cold-rolling and subsequent stress-relief annealing at $650^{\circ} \mathrm{C}$ for one hour (551-3), (3) hot-rolling followed by $20 \%$ cold-rolling (551-4), and hot-rolling only (551-5). 
TEM-10300-1

$03 / 01 / 2012$

TECHNICAL EVALUATION

Page 14 of 38

Rev. 03

Title: $\quad$ Elevated Temperature Tensile Tests on DU-10Mo Rolled Foils

$\begin{array}{llllll}\text { TEV No.: } & 2194 & \text { Rev. No.: } 2 & \text { Project No.: } 25228 & \text { Date: } 05 / 02 / 18\end{array}$

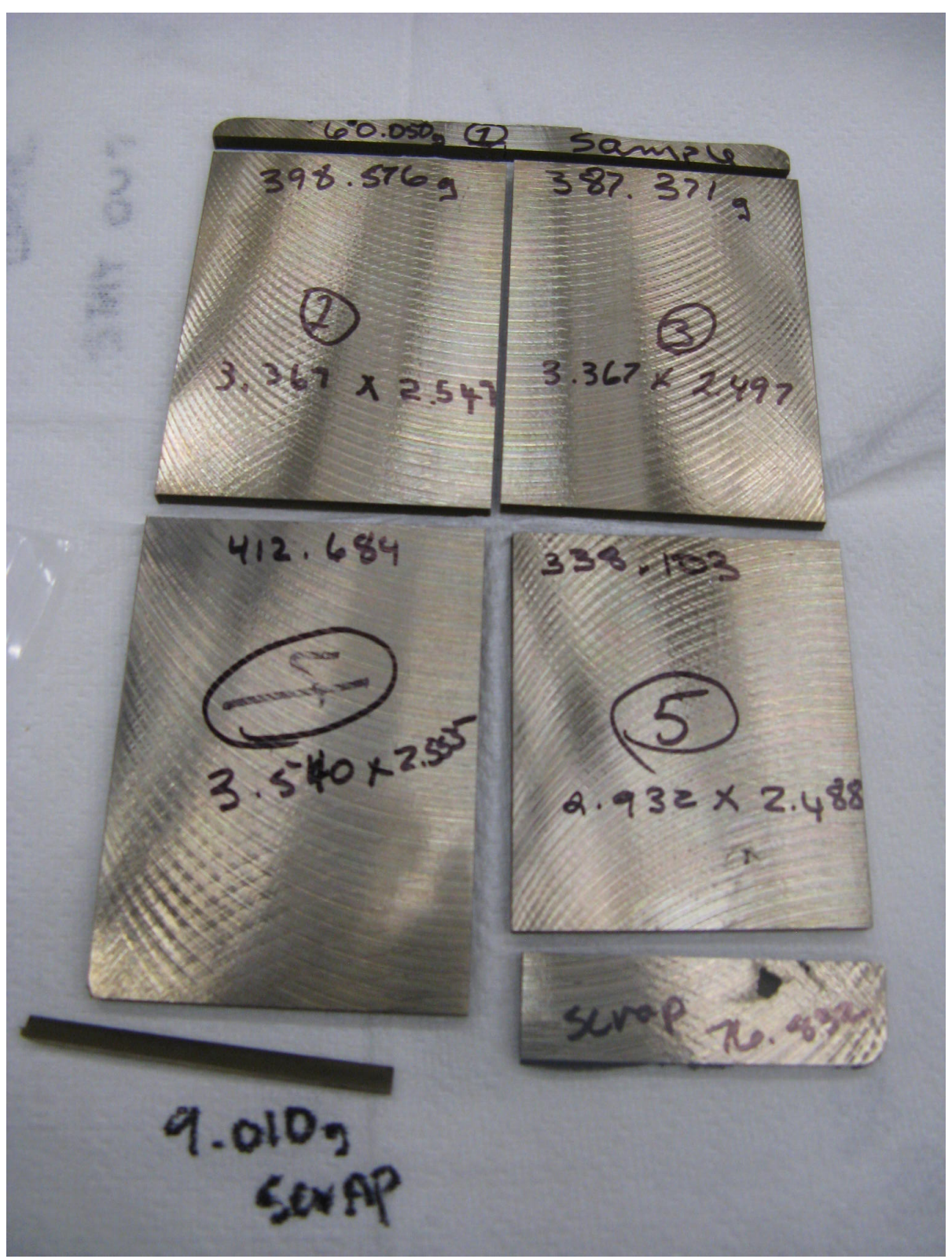

Figure 2. Machined coupon from $Y-12$ quartered into sections for rolling. Figure also shows scrap containing a visible casting defect that was cut off during sectioning.

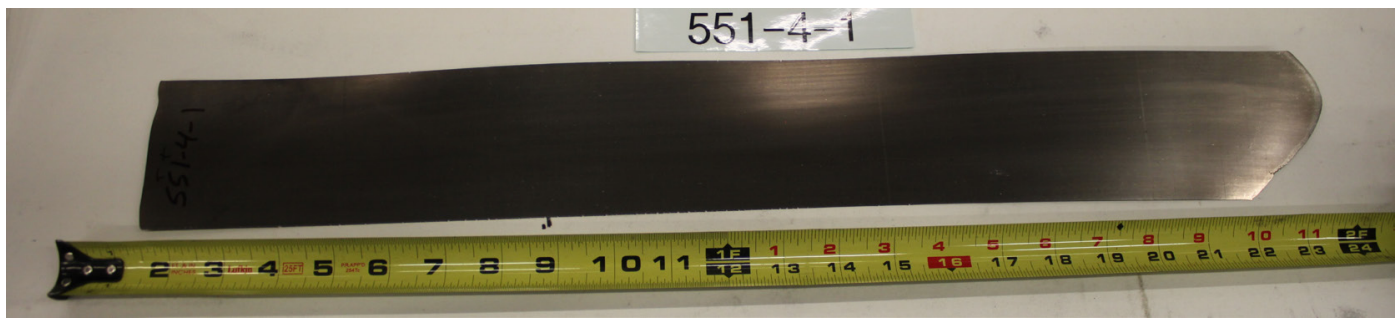

Figure 3. Foil 551-4-1 after hot rolling and cold rolling has been completed. 
Rev. 03

Title: $\quad$ Elevated Temperature Tensile Tests on DU-10Mo Rolled Foils

$\begin{array}{llllll}\text { TEV No.: } 2194 & \text { Rev. No.: } 2 & \text { Project No.: } 25228 & \text { Date: } 05 / 02 / 18\end{array}$

\section{Test Specimens and Specimen Dimensional Measurements}

Each of the four foils were further subdivided into smaller sections for handling purposes, and small tensile specimens for these tests were cut from the foil sheets using wire EDM (Figure 4, Figure 5, and Figure 6). Flat, rectangular tensile specimens were fabricated, corresponding to a reduced sub-size specimen, as described in Figure 1 in ASTM E8/8M-13, ${ }^{10}$ with an additional size reduction of $50 \%$, as shown in the INL Drawing. ${ }^{12}$ The resultant sheet-type test specimens (flat "dog bone" specimens) had nominal dimensions of overall length, $50.8 \mathrm{~mm}$; reduced section width, $3.2 \mathrm{~mm}$; and, reduced section length, $16 \mathrm{~mm}$. The small specimen size is necessary due to size limitations imposed by the furnace internal dimensions and limited material availability. Specimens were cut with the tensile axis, both parallel and transverse to the rolling direction, to assess potential effects of rolling texture on mechanical properties. Each specimen was labeled, maintaining foil ID, orientation, and location in the foil sheet from which it was removed. The specimens had gauge-mark indents placed on each specimen's lateral centerline, equidistant from the reduced section-length center, with a nominal spacing of $12.70 \mathrm{~mm}$. The marks were made with a carbide indentor that has a small radius tip and conical profile and an alignment and locating fixture. The resultant indents had a surface diameter of less than $0.08 \mathrm{~mm}$ and were uniformly circular. Figure 7, Figure 8, and Figure 9 show the marking alignment fixture and a trial specimen with marks applied.

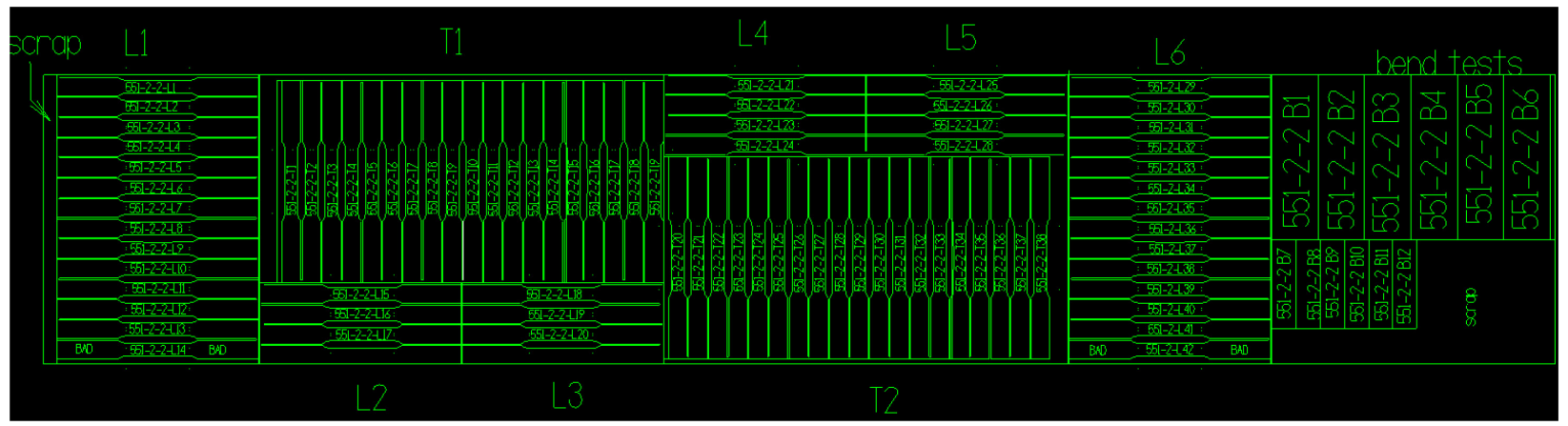

Figure 4. EDM pattern showing the cutting diagram for Foil 551-2-2.

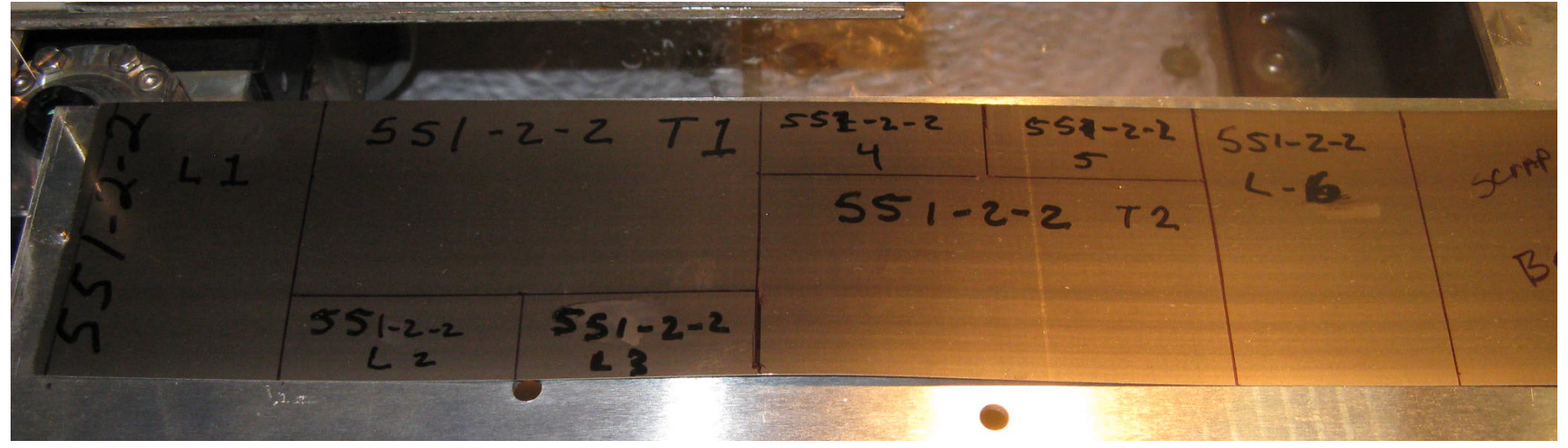

Figure 5. Foil 551-2-2 showing subsection labeling corresponding to the EDM cutting pattern. 
TEM-10300-1

$03 / 01 / 2012$

TECHNICAL EVALUATION

Page 16 of 38

Rev. 03

Title: $\quad$ Elevated Temperature Tensile Tests on DU-10Mo Rolled Foils

$\begin{array}{llllll}\text { TEV No.: } & 2194 & \text { Rev. No.: } 2 & \text { Project No.: } 25228 & \text { Date: } 05 / 02 / 18\end{array}$

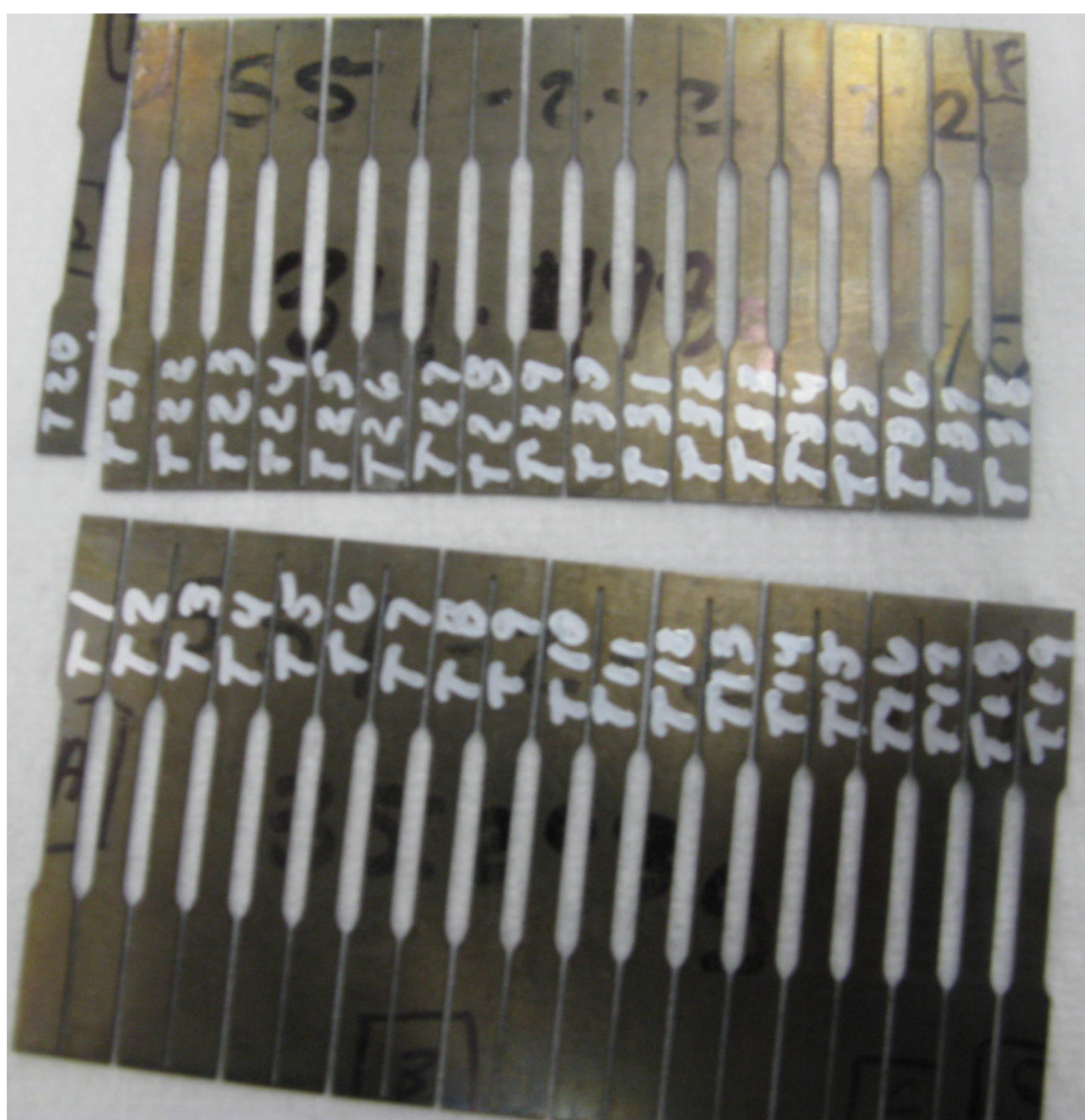

Figure 6. Individually labeled tensile specimens as cut per EDM pattern for Foil 551-2-2.

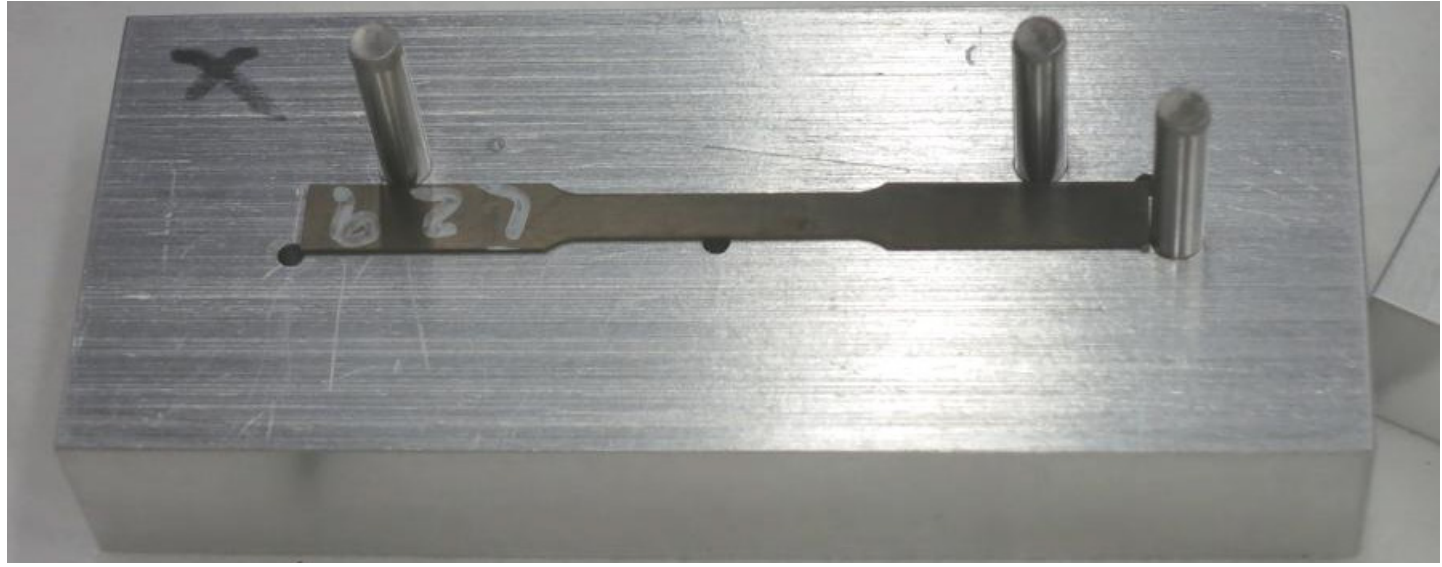

Figure 7. Gauge-marking specimen-support base. 
Rev. 03

Title: $\quad$ Elevated Temperature Tensile Tests on DU-10Mo Rolled Foils

$\begin{array}{lllll}\text { TEV No.: } 2194 & \text { Rev. No.: } 2 & \text { Project No.: } 25228 & \text { Date: } 05 / 02 / 18\end{array}$

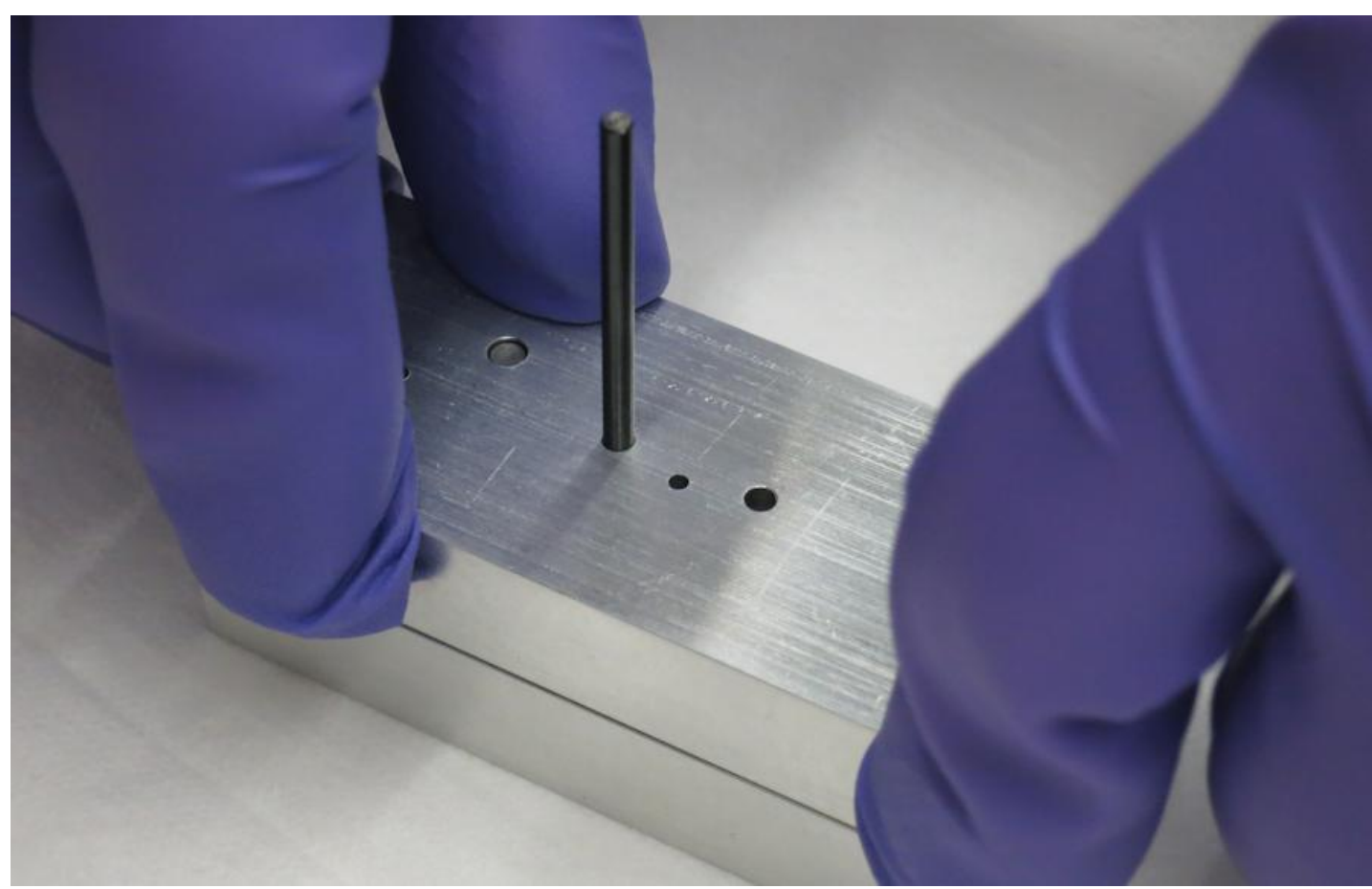

Figure 8. Marking indenter guide plate installed over specimen on support base plate. Punch is placed in one of the guide holes. Finger pressure produces a satisfactory gauge-mark indent.

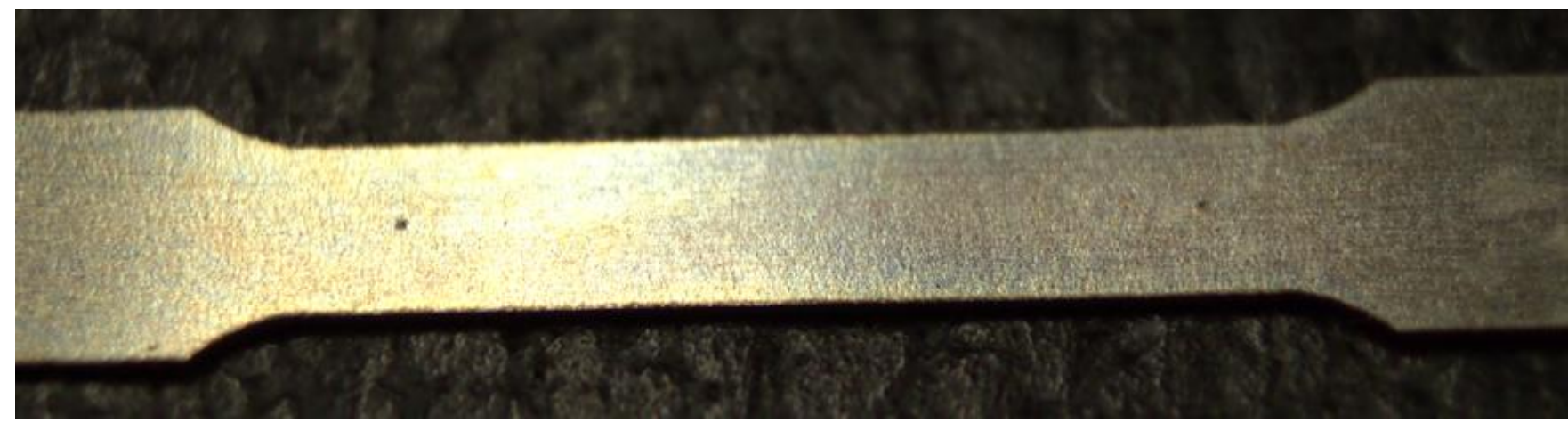

Figure 9. Stainless steel trial specimen showing gauge-mark indents produced using the marking fixture. The reduced section width is approximately $3.0 \mathrm{~mm}$. The marks shown appear larger than they actually are due to high contrast and lighting angle.

All specimens were sent to the INL MFC metrology laboratory, where width, thickness, overall length, and gauge mark spacing were measured and recorded to the nearest $0.001 \mathrm{~mm}$. Overall length for each specimen was measured to the nearest $0.01 \mathrm{~mm}$. All measurements were made with calibrated instruments. Each width and thickness measurement was made twice, and each gauge-mark-spacing measurement was made three times to improve the confidence of the measured dimensions. Locating the gauge-mark indent centers was repeatable to better than $0.005 \mathrm{~mm}$ using an optical comparator. The average pre-test dimensions for a sample of specimens from foil 551-2 are provided in Table 4. All dimensional inspections both pre-test and post-test are included in the appendix. 
Rev. 03

Title: $\quad$ Elevated Temperature Tensile Tests on DU-10Mo Rolled Foils

$\begin{array}{llllll}\text { TEV No.: } & 2194 & \text { Rev. No.: } 2 & \text { Project No.: } 25228 & \text { Date: } 05 / 02 / 18\end{array}$

Table 4. Representative average specimen pre-test dimensions from Foil 551-2.

\begin{tabular}{|c|c|c|c|c|}
\hline $\begin{array}{c}\text { Specimen } \\
\text { ID }\end{array}$ & $\begin{array}{c}\text { Thickness } \\
\text { (mm) }\end{array}$ & $\begin{array}{c}\text { Width } \\
\text { (mm) }\end{array}$ & $\begin{array}{c}\text { Gauge Mark } \\
\text { Spacing } \\
\text { (mm) }\end{array}$ & $\begin{array}{c}\text { Overall } \\
\text { Length } \\
\text { (mm) }\end{array}$ \\
\hline L-15 & 0.384 & 2.969 & 12.664 & 50.70 \\
\hline L-16 & 0.375 & 2.997 & 12.703 & 50.70 \\
\hline L-17 & 0.371 & 3.117 & 12.729 & 50.70 \\
\hline L-18 & 0.384 & 2.973 & 12.823 & 50.70 \\
\hline L-19 & 0.379 & 2.999 & 12.720 & 50.71 \\
\hline L-20 & 0.371 & 3.113 & 12.723 & 50.71 \\
\hline
\end{tabular}

All specimen dimensions were measured multiple times to ensure accuracy of measurement. Some variability in these replicate specimen dimensional inspections was noted. A brief analysis was conducted to evaluate potential errors that could transfer to the test results, as follows. Measurements from 20 randomly selected test specimens were examined. The variance of each group of replicate measurements was determined. The accumulated variances for each type of measurement were averaged, and the square root, calculated. The result is the sample standard deviation. The standard deviation of measured values from each particular measurement group is provided in Table 5 . The standard deviation for post-test thickness, width, and area are not calculated since reporting of reduction of area is not required.

Table 5. Dimensional measurement standard deviations from random sampling of 20 specimens.

\begin{tabular}{|c|c|c|c|c|}
\hline $\begin{array}{c}\text { Standard } \\
\text { Deviation }\end{array}$ & Gauge Length $(\mu \mathrm{m})$ & Thickness $(\mu \mathrm{m})$ & Width $(\mu \mathrm{m})$ & $\begin{array}{c}\text { Area } \\
\left(\mathrm{mm}^{2}\right)(\text { calculated })\end{array}$ \\
\hline Pre-Test Std.Dev. & 3.71 & 0 & 1.13 & $1.95 \mathrm{E}-6$ \\
\hline $\begin{array}{c}\text { Post-Test } \\
\text { Std.Dev. }\end{array}$ & 3.66 & & & \\
\hline
\end{tabular}

The potential error in the gauge length measurements leads to an error band for the calculated values of elongation, determined using the pre- and post-test gauge length measurement. The result, $2^{*}$ std.dev $=\sim 15 \mu \mathrm{m}$, is $\pm 0.12 \%$ of elongation at the nominal initial gauge length of $12.7 \mathrm{~mm}$.

\section{RESULTS}

Results from all of the valid tests from both longitudinal and transverse tensile specimens are presented for both room-temperature and elevated-temperature tests (see Table 6 and Table 7).

Representative stress versus strain plots for a room-temperature and a $250^{\circ} \mathrm{C}$ test are provided in Figure 14. Construction lines for determining $0.2 \%$ offset yield strength are also shown. 
Rev. 03

Title: $\quad$ Elevated Temperature Tensile Tests on DU-10Mo Rolled Foils

$\begin{array}{lllll}\text { TEV No.: } 2194 & \text { Rev. No.: } 2 & \text { Project No.: } 25228 & \text { Date: } 05 / 02 / 18\end{array}$

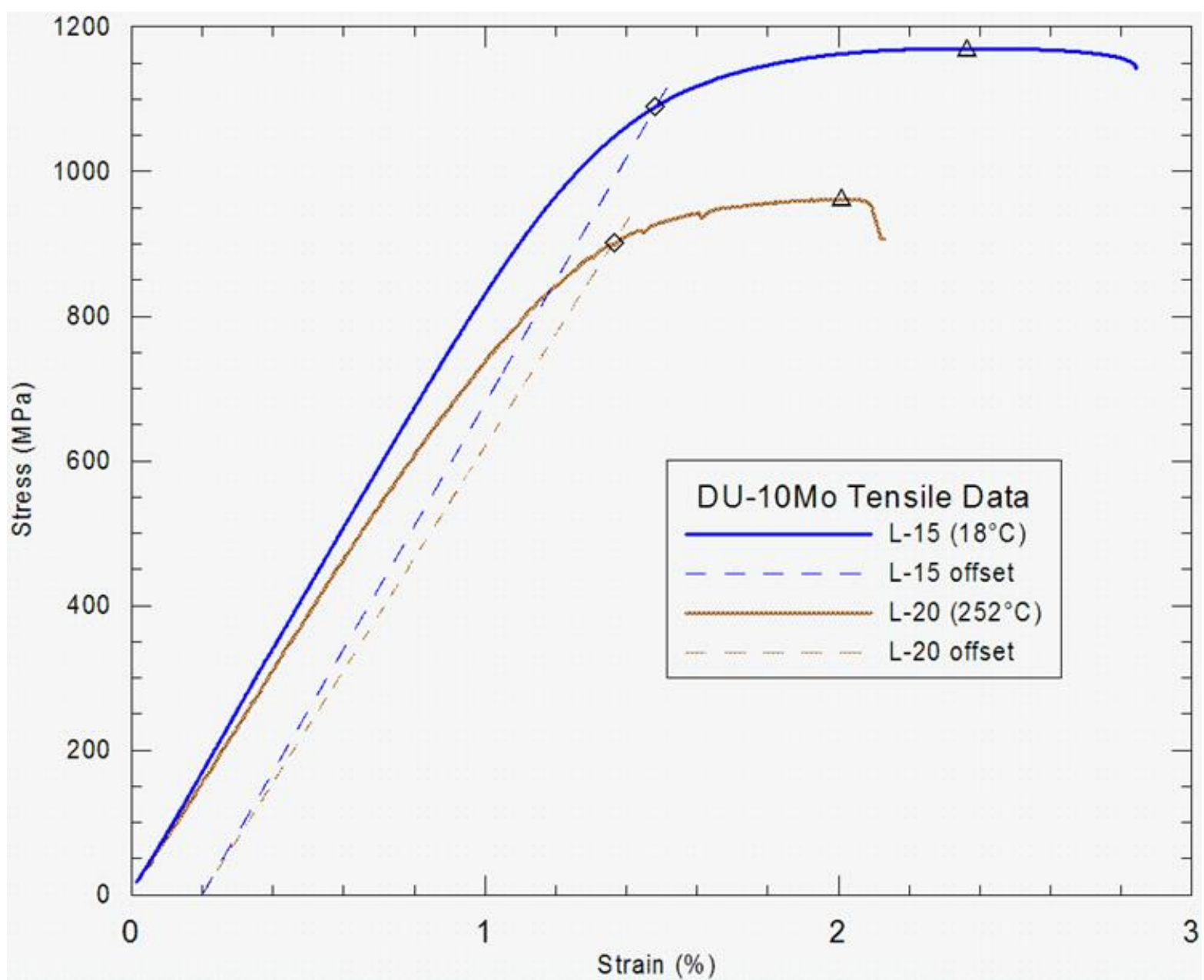

Figure 10. Stress-strain plots for room-temperature and $250^{\circ} \mathrm{C}$ tensile tests on longitudinal specimens of DU-10Mo foil $551-2$ that was prepared by hot-rolling, followed by $50 \%$ cold-rolling reduction in thickness. Slope of offset modulus lines (dashed) represents the modulus reduction expected between room temperature and $250^{\circ} \mathrm{C}$.

The mechanical properties determined by analysis of the test data, in accordance with ASTM E8/8M-13a (room-temperature) and ASTM E21-10 (elevated-temperature), are shown in Table 6 and Table 7 respectively.

\section{Summary of Required Reporting Elements}

All tests were conducted on DU-10Mo material using half-subsize sheet-type tensile specimens. Room-temperature tests were conducted in accordance with ASTM E8/8M-13a while elevated-temperatures tests were conducted using ASTM E21-10. In both cases, the $0.2 \%$ offset method was used to determine yield strength. Elongation was determined after fracture occurred. A constant crosshead speed of $0.2 \mathrm{~mm} / \mathrm{min}$ was used producing a nominal specimen strain rate of $0.5 \% / \mathrm{min}$. Calculated values were rounded up or down to the nearest digit of the required accuracy (standard rounding method), excepting that in accordance with the test standard, final specimen elongation values were rounded to the nearest $0.2 \%$ deformation interval. All elevated-temperature tests were conducted under argon atmosphere with $<20 \mathrm{ppm} \mathrm{O}_{2}$ concentration. 
TEM-10300-1

$03 / 01 / 2012$

TECHNICAL EVALUATION

Page 20 of 38

Rev. 03

Title: $\quad$ Elevated Temperature Tensile Tests on DU-10Mo Rolled Foils

$\begin{array}{lllll}\text { TEV No.: } 2194 & \text { Rev. No.: } 2 & \text { Project No.: } 25228 & \text { Date: } 05 / 02 / 18\end{array}$

In multiple cases, test results for an individual specimen were invalidated by specimen failure outside of the allowable region or, infrequently, due to other problems with a particular specimen. Replicate specimens under replicate test conditions were tested to obtain the required number of valid tests for that particular material/test condition combination.

Tests that did not fail in the middle $50 \%$ of gauge length were considered invalid and not included in results; initial fractography of a few samples of specimens indicate the failure method is ductile rupture. Some specimens failed prior to the stress-strain curve intersecting the $0.2 \%$ offset curve; these specimens do not provide a valid yield-strength value and are reported with "NA" for yield strength in the tables.

Testing equipment included the following: Instron $33665 \mathrm{kN}$ load cell, $12.7 \mathrm{~mm}$ gage length extensometer from Epsilon Corp. The extensometer has a measuring range to $+20 \%$ tensile strain meeting class B-2 requirements; CM Inc. rapid temp furnace, model 1608 (gas-sealed front loader), SN100400, Eurotherm 2404 temperature controller, thermocouple material, sheathed type-K thermocouple (calibrated) in furnace environment for temperature control, Type $\mathrm{K}$ thermocouples (calibrated) welded to the specimen grip jaw ends for temperature monitoring.

All additional information required for reporting in accordance with the testing standards is provided in Table 6 and Table 7 respectively.

Table 6. Measured mechanical properties of DU-10Mo at room temperature. Tested and reported in accordance with ASTM E8/8M-13. Material: DU-10\%Mo from plate \#551.

\begin{tabular}{|c|c|c|c|c|c|c|c|c|}
\hline $\begin{array}{l}\text { 으 } \\
\text { 훈 } \\
\text { 는 }\end{array}$ & 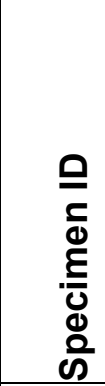 & 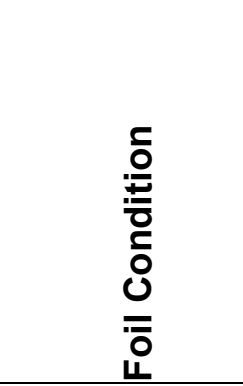 & 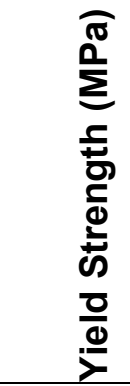 & 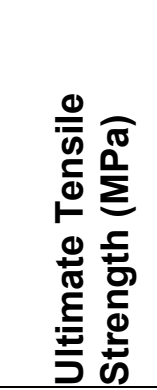 & 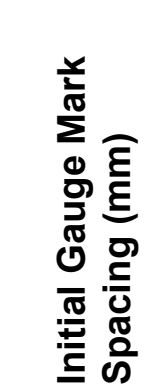 & 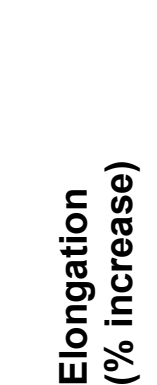 & 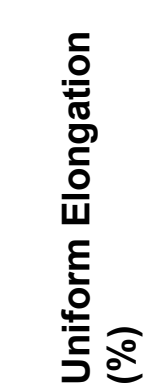 & ث̊ \\
\hline $551-2-2$ & L15 & $50 \% \mathrm{CW}$ & 1100 & 1170 & 12.664 & 1.2 & 2.36 & e. \\
\hline $551-2-2$ & L16 & $50 \% \mathrm{CW}$ & 1115 & 1179 & 12.703 & 3.6 & 2.55 & e. \\
\hline $551-2-2$ & L17 & $50 \% \mathrm{CW}$ & NA & 1168 & 12.729 & 4.0 & NA & a., b., c \\
\hline $551-2-2$ & T2 & $50 \% \mathrm{CW}$ & 1098 & 1199 & 12.675 & 2.0 & 1.97 & d. \\
\hline $551-2-2$ & T3 & $50 \% \mathrm{CW}$ & 1070 & 1070 & 12.685 & 0.2 & 1.42 & d. \\
\hline $551-2-2$ & T14 & $50 \% \mathrm{CW}$ & NA & 1064 & 12.720 & 1.6 & 1.31 & b., d. \\
\hline $551-3$ & L1 & $50 \% C W+A$ & 1013 & 1016 & 12.738 & 8.6 & 1.27 & c. \\
\hline $551-3$ & L2 & $50 \% C W+A$ & 1010 & 1012 & 12.709 & 9.0 & 1.27 & c. \\
\hline $551-3$ & L3 & $50 \% \mathrm{CW}+\mathrm{A}$ & 1013 & 1016 & 12.692 & 9.0 & 1.26 & c. \\
\hline $551-3$ & T1 & $50 \% \mathrm{CW}+\mathrm{A}$ & 1030 & 1031 & 12.678 & 7.4 & 1.33 & c. \\
\hline $551-3$ & T2 & $50 \% C W+A$ & 1030 & 1032 & 12.686 & 7.6 & 1.24 & c. \\
\hline $551-3$ & T3 & $50 \% C W+A$ & 1028 & 1028 & 12.784 & 7.0 & 1.35 & c. \\
\hline $551-4$ & L1 & $20 \% \mathrm{CW}$ & 1091 & 1139 & 12.763 & 6.2 & 2.30 & d. \\
\hline $551-4$ & L2 & $20 \% \mathrm{CW}$ & 1099 & 1148 & 12.759 & 5.8 & 2.27 & d. \\
\hline
\end{tabular}


TEM-10300-1

$03 / 01 / 2012$

TECHNICAL EVALUATION

Page 21 of 38

Rev. 03

Title: $\quad$ Elevated Temperature Tensile Tests on DU-10Mo Rolled Foils

$\begin{array}{lllll}\text { TEV No.: } 2194 & \text { Rev. No.: } 2 & \text { Project No.: } 25228 & \text { Date: } 05 / 02 / 18\end{array}$

\begin{tabular}{|c|c|c|c|c|c|c|c|c|}
\hline $\begin{array}{l}\text { 으 } \\
\overline{\overline{0}} \\
\text { 눈 }\end{array}$ & 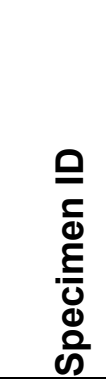 & 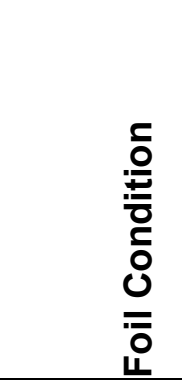 & 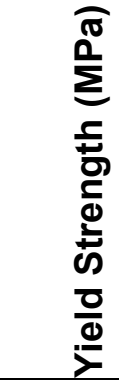 & 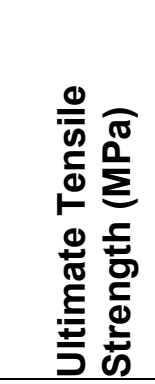 & 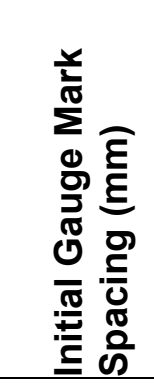 & 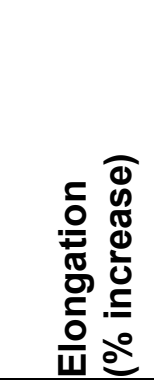 & 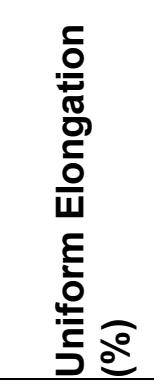 & 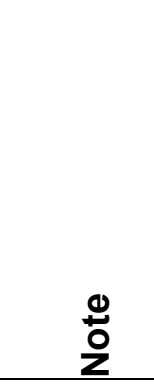 \\
\hline $551-4$ & L3 & $20 \% \mathrm{CW}$ & 1126 & 1167 & 12.758 & 5.6 & 2.24 & d. \\
\hline $551-4$ & $\mathrm{~T} 1$ & $20 \% \mathrm{CW}$ & 1058 & 1192 & 12.756 & 2.2 & 2.32 & d. \\
\hline $551-4$ & $\mathrm{~T} 2$ & $20 \% \mathrm{CW}$ & 1059 & 1109 & 12.769 & 1.6 & 1.57 & d. \\
\hline 551-4 & T3 & $20 \% \mathrm{CW}$ & 1072 & 1116 & 12.756 & 1.0 & 1.54 & d. \\
\hline $551-5$ & $\mathrm{~L} 1$ & HR Only & 1005 & 1006 & 12.730 & 10.6 & 1.29 & c. \\
\hline $551-5$ & L2 & HR Only & 1017 & 1017 & 12.715 & 12.4 & 1.38 & c. \\
\hline $551-5$ & L3 & HR Only & 1020 & 1020 & 12.696 & 12.2 & 1.32 & c. \\
\hline $551-5$ & $\mathrm{~T} 1$ & HR Only & NA & 1031 & 12.745 & 1.0 & 0.66 & b., c. \\
\hline $551-5$ & $\mathrm{~T} 2$ & HR Only & NA & 1011 & 12.725 & 1.2 & 1.08 & b., c. \\
\hline $551-5$ & T3 & HR Only & 1025 & 1026 & 12.686 & 1.4 & 1.41 & C. \\
\hline $551-5$ & T13 & HR Only & 1061 & 1063 & 12.740 & 8.0 & 1.61 & d. \\
\hline \multicolumn{9}{|c|}{$\begin{array}{l}\text { a. Specimen slipped in wedge grip jaw during early part of test, including through the zone of yielding. In-plane bending o } \\
\text { gage section is suspected to have occurred. Test was suspended, grip re-tightened, and test re-started. Subsequent } \\
\text { strain data had to be spliced with initial test data. Due to slip and bending, it was not possible to accurately connect the } \\
\text { two data sets, and an accurate } 0.2 \% \text { offset yield strength could not be established. } \\
\text { b. Specimens reached ultimate failure prior to intersection with the } 0.2 \% \text { offset curve, thus no yield strength could be } \\
\text { established. } \\
\text { c. Test operator: Jason Schulthess } \\
\text { d. Test operator: Michael Heighs } \\
\text { e. Test operator: Randy Lloyd }\end{array}$} \\
\hline
\end{tabular}

Table 7. Measured mechanical properties of DU-10Mo at elevated temperature.

\begin{tabular}{|c|c|c|c|c|c|c|c|c|c|c|}
\hline $\begin{array}{l}\text { 으 } \\
\overline{\overline{0}} \\
\text { 는 }\end{array}$ & 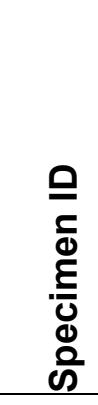 & 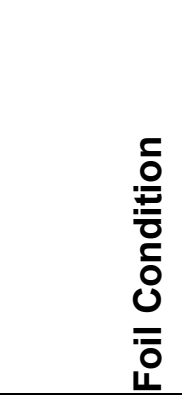 & $\begin{array}{l}0 \\
0 \\
0 \\
\frac{0}{3} \\
\frac{\pi}{0} \\
\frac{0}{d} \\
\frac{0}{E} \\
\frac{0}{0} \\
\end{array}$ & 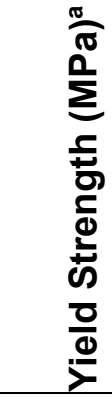 & 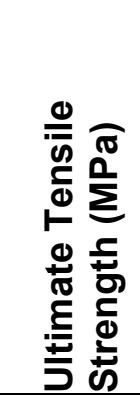 & 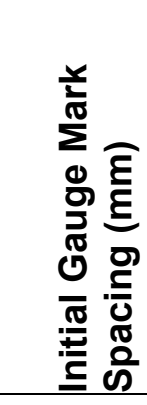 & 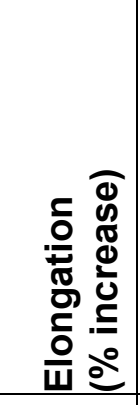 & 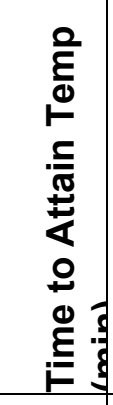 & 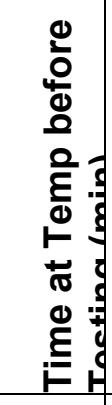 & 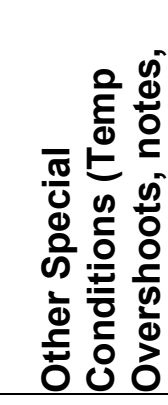 \\
\hline $551-2-2$ & L18 & $50 \% \mathrm{CW}$ & 250 & 837 & 952 & 12.823 & 3.0 & 160 & 30 & e. \\
\hline $551-2-2$ & L20 & $50 \% \mathrm{CW}$ & 250 & 880 & 962 & 12.723 & 3.6 & 150 & 5 & c. \\
\hline $551-2-2$ & L22 & $50 \% \mathrm{CW}$ & 250 & 887 & 909 & 12.721 & 1.6 & 120 & 10 & C. \\
\hline $551-2-2$ & T11 & $50 \% \mathrm{CW}$ & 250 & NA & 820 & 12.700 & 1.8 & 275 & 10 & b., c. \\
\hline
\end{tabular}


TEM-10300-1

$03 / 01 / 2012$

TECHNICAL EVALUATION

Page 22 of 38

Rev. 03

Title: $\quad$ Elevated Temperature Tensile Tests on DU-10Mo Rolled Foils

$\begin{array}{lllll}\text { TEV No.: } 2194 & \text { Rev. No.: } 2 & \text { Project No.: } 25228 & \text { Date: } 05 / 02 / 18\end{array}$

\begin{tabular}{|c|c|c|c|c|c|c|c|c|c|c|}
\hline $\begin{array}{l}\text { 으 } \\
\text { 훈 } \\
\text { 는 }\end{array}$ & 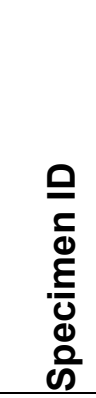 & 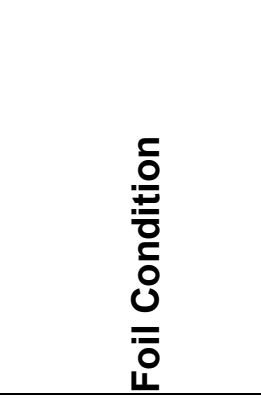 & 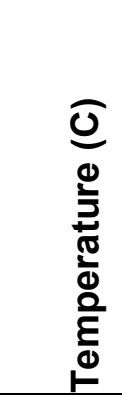 & $\begin{array}{l}\frac{\sigma}{\sigma} \\
\frac{0}{\Sigma} \\
\frac{0}{5} \\
\frac{0}{0} \\
\frac{0}{0} \\
\frac{0}{0} \\
\frac{0}{0} \\
\frac{0}{0}\end{array}$ & 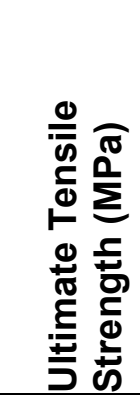 & 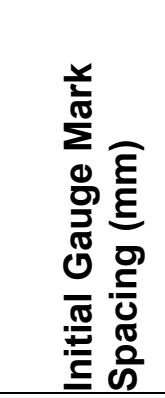 & 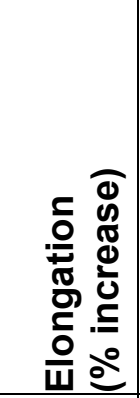 & 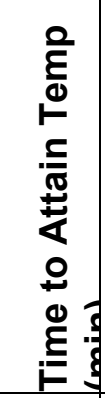 & 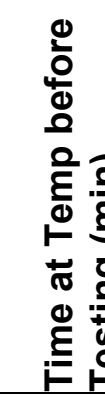 & 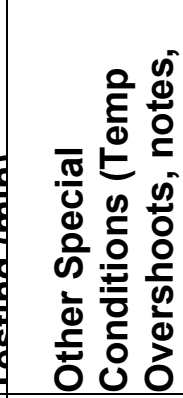 \\
\hline $551-2-2$ & $\mathrm{~T} 12$ & $50 \% \mathrm{CW}$ & 250 & 864 & 872 & 12.785 & 1.2 & 160 & 5 & c. \\
\hline $551-2-2$ & $\mathrm{~T} 13$ & $50 \% \mathrm{CW}$ & 250 & 856 & 888 & 12.727 & 1.8 & 120 & 5 & c. \\
\hline $551-2-2$ & L2 & $50 \% \mathrm{CW}$ & 350 & 821 & 884 & 12.706 & 4.5 & 100 & 5 & c. \\
\hline $551-2-2$ & L3 & $50 \% \mathrm{CW}$ & 350 & 765 & 876 & 12.765 & 3.8 & 90 & 5 & d. \\
\hline $551-2-2$ & T5 & $50 \% \mathrm{CW}$ & 350 & NA & 672 & 12.746 & 1.0 & 90 & 10 & b., c. \\
\hline $551-2-2$ & $\mathrm{~T} 7$ & $50 \% \mathrm{CW}$ & 350 & 513 & 513 & 12.727 & 0.2 & 90 & 30 & d. \\
\hline $551-2-2$ & L4 & $50 \% \mathrm{CW}$ & 450 & 539 & 612 & 12.691 & 9.6 & 90 & 10 & c. \\
\hline $551-2-2$ & L6 & $50 \% \mathrm{CW}$ & 450 & 501 & 599 & 12.766 & 8.4 & 90 & 5 & c. \\
\hline $551-2-2$ & L8 & $50 \% \mathrm{CW}$ & 550 & 141 & 175 & 12.735 & 73.8 & 120 & 10 & c. \\
\hline $551-2-2$ & L9 & $50 \% \mathrm{CW}$ & 550 & 136 & 169 & 12.741 & 81.8 & 90 & 5 & c. \\
\hline $551-2-2$ & L10 & $50 \% \mathrm{CW}$ & 550 & 136 & 162 & 12.756 & 64.2 & 120 & 10 & c. \\
\hline $551-2-2$ & T8 & $50 \% \mathrm{CW}$ & 550 & 81 & 108 & 12.686 & 51.0 & 120 & 40 & d., f. \\
\hline $551-2-2$ & T9 & $50 \% \mathrm{CW}$ & 550 & 108 & 164 & 12.788 & 36.4 & 160 & 10 & d. \\
\hline $551-2-2$ & $\mathrm{~T} 10$ & $50 \% \mathrm{CW}$ & 550 & 109 & 159 & 12.796 & 39.2 & 100 & 5 & c. \\
\hline $551-3$ & L4 & $50 \% \mathrm{CW}+\mathrm{A}$ & 200 & 673 & 712 & 12.756 & 13.4 & 180 & 5 & c. \\
\hline $551-3$ & $\mathrm{~L} 5$ & $50 \% C W+A$ & 200 & 653 & 708 & 12.694 & 13.0 & 120 & 5 & c. \\
\hline $551-3$ & L6 & $50 \% C W+A$ & 200 & 661 & 710 & 12.674 & 14.2 & 120 & 5 & d. \\
\hline $551-3$ & $\mathrm{~T} 4$ & $50 \% \mathrm{CW}+\mathrm{A}$ & 200 & 677 & 722 & 12.714 & 12.0 & 105 & 5 & c. \\
\hline $551-3$ & T5 & $50 \% C W+A$ & 200 & 681 & 728 & 12.744 & 16.0 & 120 & 5 & c. \\
\hline $551-3$ & T6 & $50 \% C W+A$ & 200 & 676 & 724 & 12.693 & 12.2 & 110 & 5 & c. \\
\hline $551-3$ & $\mathrm{~L} 7$ & $50 \% C W+A$ & 400 & 491 & 569 & 12.736 & 3.6 & 90 & 5 & d. \\
\hline $551-3$ & L13 & $50 \% C W+A$ & 400 & 483 & 559 & 12.715 & 5.8 & 80 & 5 & d. \\
\hline $551-3$ & L29 & $50 \% C W+A$ & 400 & 489 & 561 & 12.713 & 5.4 & 100 & 5 & d. \\
\hline $551-3$ & T8 & $50 \% \mathrm{CW}+\mathrm{A}$ & 400 & 493 & 576 & 12.640 & 5.2 & 90 & 10 & c. \\
\hline $551-3$ & T9 & $50 \% C W+A$ & 400 & 493 & 569 & 12.628 & 6.2 & 90 & 10 & c. \\
\hline 551-3 & T13 & $50 \% C W+A$ & 400 & 505 & 578 & 12.720 & 6.2 & 100 & 5 & c. \\
\hline
\end{tabular}


TEM-10300-1

$03 / 01 / 2012$

TECHNICAL EVALUATION

Page 23 of 38

Rev. 03

Title: $\quad$ Elevated Temperature Tensile Tests on DU-10Mo Rolled Foils

$\begin{array}{lllll}\text { TEV No.: } 2194 & \text { Rev. No.: } 2 & \text { Project No.: } 25228 & \text { Date: } 05 / 02 / 18\end{array}$

\begin{tabular}{|c|c|c|c|c|c|c|c|c|c|c|}
\hline $\begin{array}{l}\text { 으 } \\
\overline{\overline{0}} \\
\text { ㄴ }\end{array}$ & 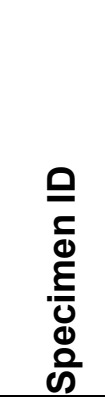 & 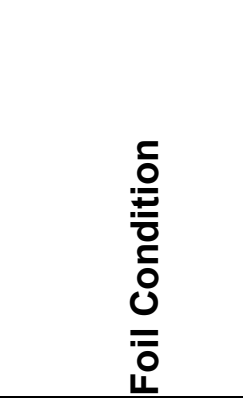 & 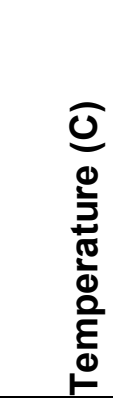 & 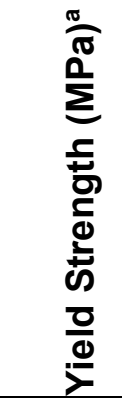 & 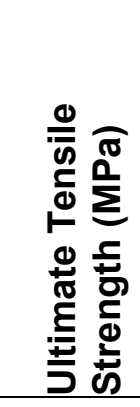 & 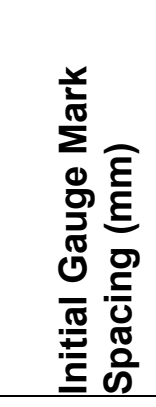 & 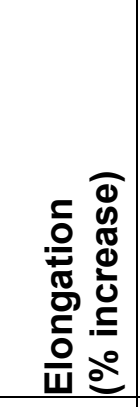 & 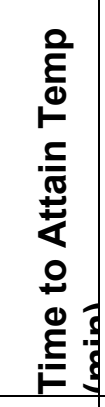 & 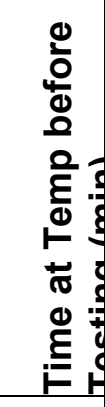 & 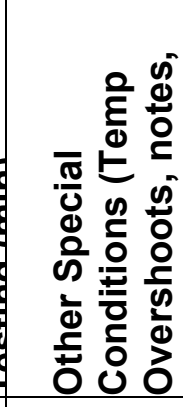 \\
\hline $551-3$ & L10 & $50 \% C W+A$ & 550 & 161 & 200 & 12.730 & 68.6 & 80 & 5 & c. \\
\hline $551-3$ & L11 & $50 \% C W+A$ & 550 & 162 & 197 & 12.774 & 61.4 & 90 & 5 & d. \\
\hline $551-3$ & L12 & $50 \% C W+A$ & 550 & 174 & 202 & 12.689 & 77.2 & 120 & 5 & c. \\
\hline $551-3$ & $\mathrm{~T} 10$ & $50 \% C W+A$ & 550 & 161 & 192 & 12.856 & 68.0 & 100 & 5 & d. \\
\hline $551-3$ & T11 & $50 \% C W+A$ & 550 & 165 & 204 & 12.732 & 62.4 & 100 & 5 & d. \\
\hline $551-3$ & $\mathrm{~T} 12$ & $50 \% C W+A$ & 550 & 161 & 195 & 12.729 & 66.6 & 80 & 5 & d. \\
\hline $551-4$ & L4 & $20 \% \mathrm{CW}$ & 200 & 867 & 911 & 12.776 & 3.0 & 225 & 5 & c., f. \\
\hline $551-4$ & L5 & $20 \% \mathrm{CW}$ & 200 & 875 & 917 & 12.851 & 2.8 & 210 & 10 & d. \\
\hline $551-4$ & L6 & $20 \% \mathrm{CW}$ & 200 & 866 & 913 & 12.729 & 3.0 & 185 & 10 & d. \\
\hline $551-4$ & $\mathrm{~T} 4$ & $20 \% \mathrm{CW}$ & 200 & 780 & 954 & 12.721 & 4.0 & 120 & 5 & c. \\
\hline $551-4$ & T5 & $20 \% \mathrm{CW}$ & 200 & 800 & 953 & 12.778 & 3.6 & 140 & 10 & c. \\
\hline $551-4$ & $\mathrm{T7}$ & $20 \% \mathrm{CW}$ & 200 & 816 & 895 & 12.748 & 1.6 & 135 & 15 & c. \\
\hline $551-4$ & L7 & $20 \% \mathrm{CW}$ & 400 & 611 & 719 & 12.697 & 2.8 & 80 & 5 & c. \\
\hline $551-4$ & L8 & $20 \% \mathrm{CW}$ & 400 & 621 & 711 & 12.744 & 2.4 & 80 & 10 & c. \\
\hline $551-4$ & L10 & $20 \% \mathrm{CW}$ & 550 & 146 & 185 & 12.717 & 50.6 & 220 & 5 & d. \\
\hline $551-4$ & L11 & $20 \% \mathrm{CW}$ & 550 & 150 & 193 & 12.731 & 43.8 & 150 & 5 & d. \\
\hline $551-4$ & L13 & $20 \% \mathrm{CW}$ & 550 & 160 & 200 & 12.797 & 43.4 & 130 & 10 & c. \\
\hline $551-4$ & T10 & $20 \% \mathrm{CW}$ & 550 & 156 & 198 & 12.779 & 25.4 & 90 & 5 & d. \\
\hline $551-4$ & T11 & $20 \% \mathrm{CW}$ & 550 & 148 & 180 & 12.719 & 43.0 & 150 & 5 & c. \\
\hline $551-4$ & $\mathrm{~T} 12$ & $20 \% \mathrm{CW}$ & 550 & 156 & 198 & 12.713 & 32.8 & 110 & 5 & c. \\
\hline $551-5$ & L4 & HR Only & 200 & 660 & 732 & 12.849 & 13.0 & 220 & 20 & d. \\
\hline $551-5$ & L5 & HR Only & 200 & 665 & 701 & 12.690 & 4.8 & 140 & 5 & d. \\
\hline $551-5$ & L6 & HR Only & 200 & 664 & 719 & 12.769 & 6.4 & 125 & 10 & c. \\
\hline $551-5$ & $\mathrm{~T} 4$ & HR Only & 200 & 695 & 710 & 12.691 & 2.0 & 120 & 5 & d. \\
\hline $551-5$ & T5 & HR Only & 200 & 701 & 733 & 12.744 & 2.9 & 120 & 10 & c. \\
\hline $551-5$ & T6 & HR Only & 200 & 691 & 725 & 12.747 & 4.8 & 120 & 10 & c. \\
\hline
\end{tabular}


Rev. 03

Title:

\begin{tabular}{|l|l|l|l|l|l|l|l|l|l|l|}
\hline & & & & & & & & \\
\end{tabular}

Definitions of reported properties are provided in ASTM E8/8M-13.

Given the small size of the test specimens, which tend to increase specimen-to-specimen variation in measured properties, the results are reasonably consistent.

Tensile properties of $\mathrm{DU}-10 \mathrm{Mo}$ at room temperature through approximately $400^{\circ} \mathrm{C}$ determined from the tests conducted herein suggest the material is stronger and has lower ductility than what has been reported previously in the literature. The explanation for these differences has yet to be determined, but is likely related to differences in grain size and/or impurity content. At the highest temperatures tested $\left(550^{\circ} \mathrm{C}\right)$ better agreement between the values reported here and available literature was found. As expected, yield and ultimate tensile strength decreased with increasing test temperature. Generally, the yield stress for all foil processing conditions was found to be in the range of $1100 \mathrm{MPa}$ for room temperature tests, and in the range of $200 \mathrm{MPa}$ for tests conducted at $550^{\circ} \mathrm{C}$. Ultimate tensile stress was in the range of $1175 \mathrm{MPa}$ at room temperature, decreasing to approximately $225 \mathrm{MPa}$ at $550^{\circ} \mathrm{C}$. Elongation increased significantly from $1-2 \%$ at room temperature, to $50 \%$ or more for the tests at $550^{\circ} \mathrm{C}$. Additional details on the observed effects of foil processing condition and specimen orientation on tensile properties are summarized below: 
Rev. 03

Title: $\quad$ Elevated Temperature Tensile Tests on DU-10Mo Rolled Foils

$\begin{array}{lllll}\text { TEV No.: } 2194 & \text { Rev. No.: } 2 & \text { Project No.: } 25228 & \text { Date: } 05 / 02 / 18\end{array}$

\section{Yield Strength}

No significant effect of fabrication history on yield stress was observed at the lowest temperature (room temperature) and highest temperature $\left(550^{\circ} \mathrm{C}\right)$ tested. However, tests indicated yield strength differences exist at the intermediate temperatures tested with the $50 \%$ cold worked and annealed, and hot rolled only material producing lower yield stress at the intermediate test temperatures. Significant effects of specimen orientation on yield strength were only observed in a few cases (specifically $20 \%$ cold worked material tested at $200^{\circ} \mathrm{C}$ resulted in lower yield stress in the transvers orientation, and hotrolled only material tested at room temperature and $200^{\circ} \mathrm{C}$ where the transvers orientation resulted in slightly higher yield sress).

\section{Ultimate Tensile Strength (UTS)}

Significant differences in the UTS were noted in the longitudinal direction for the four foils at room temperature, with the difference continuing, but converging as the temperature increased. This difference between each of the four foils was also found to exist in the transvers direction. The $50 \%$ cold worked and annealed, and hot rolled only material produced lower ultimate tensile stress at room and the intermediate test temperatures, but converged when tested at $550^{\circ} \mathrm{C}$.

Orientation effects for specimens from the same foil were only noted in the following case: $50 \%$ cold worked at $350^{\circ} \mathrm{C}$ in which the transvers direction produced lower ultimate tensile stress. All other tests indicated no significant anisotropy due to rolling direction.

\section{Slope}

Slope of the initial section of the stress-strain curve was calculated for each specimen and then averaged across all specimens tested in each temperature group. The average slope in $\mathrm{MPa} / \%$ and standard deviation for each test temperature are: $20^{\circ} \mathrm{C}: 884.21 \pm 13.15 ; 200-250^{\circ} \mathrm{C}$ : $417.63 \pm 16.81$; $350^{\circ} \mathrm{C}: 422.25 \pm 14.75 ; 400-450^{\circ} \mathrm{C}: 320.43 \pm 17.88 ; 550^{\circ} \mathrm{C}: 199.29 \pm 24.41$. Room-temperature elastic-modulus values reported in the literature are in the low-to-mid-80s GPa range ${ }^{14,15}$ _consistent with our room-temperature test results.

\section{Ductility}

Room-temperature ductility was determined for all of the foil conditions tested. Of note, the $50 \%$ cold worked and annealed foil (foil 551-3) showed increased ductility compared to the $50 \%$ and $20 \%$ cold worked foils. The hot rolled only foil showed more ductility in the longitudinal direction than any other foil at room temperature, but showed very little ductility in the transverse direction, indicating significant anisotropy at room temperature.

Ductility increased continuously for all foil conditions as testing temperature increased, and was significant for foils tested at $550^{\circ} \mathrm{C}$. At $550^{\circ} \mathrm{C}$, the ductility for all foil conditions and orientations increased from $\sim<10 \%$ to as much as $\sim 70 \%$ in the $50 \%$ cold worked foil in the longitudinal direction (551-2) and also $~ 70 \%$ for the $50 \%$ cold worked and annealed foil (551-3) in both directions. The increase in ductility at $550^{\circ} \mathrm{C}$ was more pronounced for the $20 \%$ cold worked foil than for other foil processing conditions.

For a few specimens in both room temperature and elevated temperature cases, the $0.2 \%$ offset curve did not intersect the stress strain curve. Theoretically, ductility would be very small in these cases. When combined with the measurement error evaluated in Table 5, and recognizing that it can be 
Rev. 03

Title: $\quad$ Elevated Temperature Tensile Tests on DU-10Mo Rolled Foils

$\begin{array}{lllll}\text { TEV No.: } 2194 & \text { Rev. No.: } 2 & \text { Project No.: } 25228 & \text { Date: } 05 / 02 / 18\end{array}$

difficult to accurately piece specimens back together for post-test elongation measurements due to roughness at the fracture surface; it is likely that measurements due to roughness at the fracture surface; it is likely that actual elongation values are less than the reported values of $3 \%$ or less. This specifically includes the following specimens tested at room temperature: 551-2-2 L17, and T14; 551-5 T1, and T2; and the following specimens tested at elevated temperature: 551-2-2 T11, and T5, but may impact any specimens with reported elongation values of less than $3 \%$.

\section{Recommendations for Future Work}

It is recommended that future work include fractography of selected specimens to determine if failure mechanisms other than ductile rupture exist, and whether fracture initiation sites can be identified. Metallography and microstructural characterization should be completed to characterize grain sizes and other microstructural features that may explain the observed mechanical behavior. Finally, it is recommended that additional testing be conducted on similarly processed material having different impurity content (particularly different carbide distributions) to better understand the range of properties that may be expected in commercially fabricated fuel foils.

The room-temperature properties appear different from the expected ranges based upon historical data according to [4], [5], and [6], but it is noted that both source material chemistry and thermo-mechanical processing history of the test specimens can result in significant microstructural differences that may explain these results. ${ }^{16}$ Further characterization work is therefore needed to better understand the differences in reported properties.

\section{DISCUSSION}

Test-system compliance (including test frame components, pull rods (long), and high-temperature wedge grips) was assessed prior to commencing this series of tests. Machine crosshead displacement was converted to effective grip displacement using a quadratic compliance-correction function for the elevated-temperature tests. This assessment is discussed in [7]. The compliance-corrected grip displacement was used to estimate reduced section strain in the specimen. Slope of the initial section of the stress-strain curve was calculated for each specimen and then averaged across all specimens tested in each temperature group. Thus, the average slope in $\mathrm{MPa} / \%$ and standard deviation by test temperature group are: $20^{\circ} \mathrm{C}: 884.21 \pm 13.15 ; 200-250^{\circ} \mathrm{C}: 417.63 \pm 16.81 ; 350^{\circ} \mathrm{C}: 422.25 \pm 14.75 ; 400-$ $450^{\circ} \mathrm{C}: 320.43 \pm 17.88 ; 550^{\circ} \mathrm{C}: 199.29 \pm 24.41$. Since tension testing per ASTM E111-04 was not conducted and the tests do not meet the requirements of ASTM E111-04 ${ }^{17}$, the slope values reported here are only instructive to provide a comparison to reported elastic modulus values. Historically, room-temperature elastic-modulus values were reported to be in the low-to-mid-80s GPa range ${ }^{18,19}$ consistent with our room-temperature test results for slope of the initial section of the stress-strain curve. Additional modulus data for various temperatures were found in [4] and are summarized in Table 1. Since some non-linearity was noted in the very early stages of the stress-strain curves (particularly at higher temperatures), it is recommended, if desirable to achieve a more accurate measurement, that modulus be evaluated using ASTM E494-10. ${ }^{20}$

\section{Calculated Strength Uncertainties}

The overall uncertainty in specimen strength calculations is a function of accuracy of specimen pre-test dimensional measurement (inconsequential in these tests) and measured force errors (described above). Additionally, linearity of strain transducer response and, to a lesser degree, the absolute accuracy of the measured strain values influences the yield strength determinations. 
Rev. 03

Title: $\quad$ Elevated Temperature Tensile Tests on DU-10Mo Rolled Foils

$\begin{array}{lllll}\text { TEV No.: } & 2194 & \text { Rev. No.: } 2 & \text { Project No.: } 25228 & \text { Date: } 05 / 02 / 18\end{array}$

Standard deviation for yield stress and ultimate tensile stress for each group of specimens was calculated. The standard deviation of calculated strength values for replicate specimen groups are reported in Table 8. Standard deviations are typically 8-14 MPa, with a low value of zero (perfect specimen-to-specimen agreement); two UTS groups were approximately 65-80 MPa. In essence, replicate specimen-to-specimen variability seemed to be larger than combined errors introduced by force-measurement inaccuracies.

The test results show good specimen-to-specimen consistency with the exception of transverse tests of foil 551-5 at room temperature. In this case, the results of one specimen were further than one standard deviation below the other tests. One additional test was performed for this condition, which agreed with the primary cluster of results and implies that the one test with low-strength results may have had an uncharacteristic failure mechanism causing premature failure.

Tests were conducted using a constant cross head displacement rate of $0.2 \mathrm{~mm} / \mathrm{min}$ resulting in a strain rate of approximately $0.5 \% / \mathrm{min}$. At the higher temperatures tested, some strain rate effect may be present. The lack of linearity in the early portion of the test data for the high temperature tests $\left(550^{\circ} \mathrm{C}\right)$, suggests that the rate of stress relaxation and is close to the stress induction rate, at the tested strain rate.

A summary of the test results is presented in Table 8. Mechanical properties as a function of temperature within the range of room temperature to $550^{\circ} \mathrm{C}$ are shown graphically in Figure 11 through Figure 22. Figure 11 through Figure 22 indicate little to no fabrication effect on yield stress on the lowest temperature (room temperature) and highest temperature $\left(550^{\circ} \mathrm{C}\right)$ tested. Figure 11 through Figure 14 do indicate divergence in the intermediate temperatures tested based on fabrication effects. Effects of orientation (anisotropy due to rolling) only seem to appear in the following yield stress cases $\left(50 \%\right.$ cold worked at $250^{\circ} \mathrm{C}$ and $350^{\circ} \mathrm{C}, 20 \%$ cold worked at $200^{\circ} \mathrm{C}$, and hot-rolled only at room temp and $200^{\circ} \mathrm{C}$ ) (see Figure 15 through Figure 22). However, these orientation effects are not large in magnitude. Similar orientation effects are noted in the ultimate tensile stress results as shown in Figure 15 through Figure 22. Reviewing the data in Table 8, we note that post cold rolling annealing heat treatment on Foil 551-3 produces results (yield stress, ultimate tensile stress) very similar to the hot rolled only foil (551-5), suggesting this post-cold rolling thermal treatment is effective at substantially recovering tensile properties comparable to hot-rolled only material.

Table 8. Summary of mechanical properties from the current work.

\begin{tabular}{|c|c|c|c|c|c|c|c|c|}
\hline 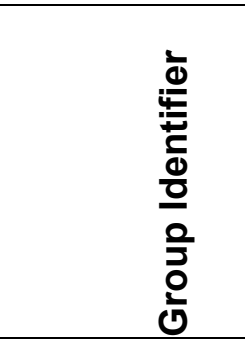 & 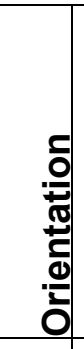 & 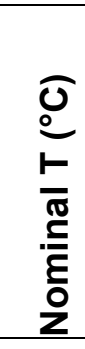 & 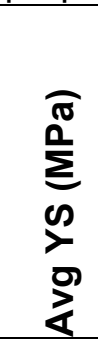 & 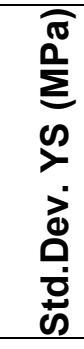 & 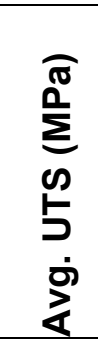 & 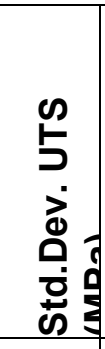 & $\begin{array}{l}\frac{}{0} \\
\frac{0}{\pi} \\
\frac{0}{0} \\
\frac{1}{0} \\
\frac{0}{4} \\
0 \\
0\end{array}$ & 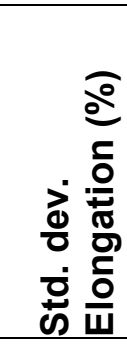 \\
\hline 551-2-2-L-20 & $\mathrm{L}$ & 20 & 1108 & 8 & 1172 & 5 & 2.9 & $1.2 \%$ \\
\hline $551-2-2-T-20$ & $\mathrm{~T}$ & 20 & 1084 & 14 & 1111 & 62 & 1.2 & $0.8 \%$ \\
\hline 551-3-L-20 & $\mathrm{L}$ & 20 & 1012 & 1 & 1015 & 1 & 8.9 & $0.2 \%$ \\
\hline 551-3-T-20 & $\mathrm{T}$ & 20 & 1029 & 1 & 1030 & 2 & 7.3 & $0.3 \%$ \\
\hline 551-4-L-20 & $\mathrm{L}$ & 20 & 1105 & 15 & 1151 & 12 & 5.9 & $0.2 \%$ \\
\hline
\end{tabular}


TEM-10300-1

$03 / 01 / 2012$

TECHNICAL EVALUATION

Page 28 of 38

Rev. 03

Title: $\quad$ Elevated Temperature Tensile Tests on DU-10Mo Rolled Foils

$\begin{array}{llllll}\text { TEV No.: } & 2194 & \text { Rev. No.: } 2 & \text { Project No.: } 25228 & \text { Date: } 05 / 02 / 18\end{array}$

\begin{tabular}{|c|c|c|c|c|c|c|c|c|}
\hline 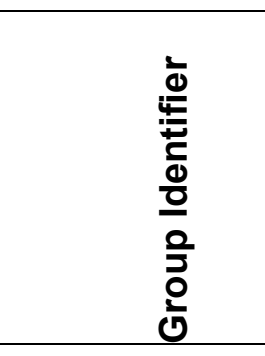 & 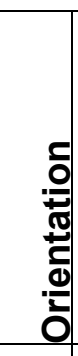 & 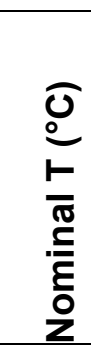 & 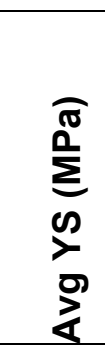 & 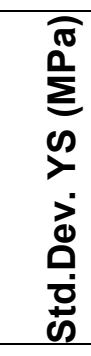 & $\begin{array}{l}\frac{\pi}{2} \\
\frac{1}{\Sigma} \\
0 \\
5 \\
5 \\
\vdots \\
\vdots\end{array}$ & 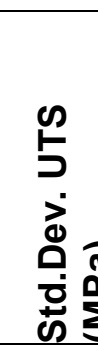 & 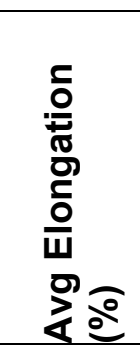 & 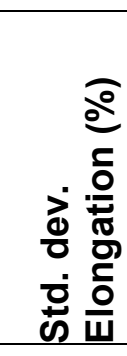 \\
\hline 551-4-T-20 & $\mathrm{T}$ & 20 & 1063 & 6 & 1139 & 38 & 1.6 & $0.5 \%$ \\
\hline 551-5-L-20 & $\mathrm{L}$ & 20 & 1043 & 18 & 1014 & 6 & 11.7 & $0.8 \%$ \\
\hline 551-5-T-20 & $\mathrm{T}$ & 20 & 1025 & 0 & 1033 & 19 & 2.9 & $2.9 \%$ \\
\hline 551-3-L-200 & L & 200 & 662 & 8 & 710 & 2 & 13.5 & $0.5 \%$ \\
\hline 551-3-T-200 & $\mathrm{T}$ & 200 & 678 & 2 & 725 & 2 & 13.4 & $1.8 \%$ \\
\hline 551-4-L-200 & $\mathrm{L}$ & 200 & 869 & 4 & 914 & 2 & 2.9 & $0.1 \%$ \\
\hline 551-4-T-200 & $\mathrm{T}$ & 200 & 799 & 15 & 934 & 28 & 3.1 & $1.0 \%$ \\
\hline 551-5-L-200 & $\mathrm{L}$ & 200 & 663 & 2 & 717 & 13 & 8.1 & $3.6 \%$ \\
\hline 551-5-T-200 & $\mathrm{T}$ & 200 & 696 & 4 & 723 & 10 & 3.2 & $1.2 \%$ \\
\hline 551-2-2-L-250 & $\mathrm{L}$ & 250 & 868 & 22 & 941 & 23 & 2.7 & $0.8 \%$ \\
\hline 551-2-2-T-250 & $\mathrm{T}$ & 250 & 860 & 4 & 860 & 29 & 1.6 & $0.3 \%$ \\
\hline 551-2-2-L-350 & L & 350 & 793 & 28 & 880 & 4 & 4.1 & $0.3 \%$ \\
\hline 551-2-2-T-350 & $\mathrm{T}$ & 350 & NA & NA & 593 & 80 & 0.6 & $0.4 \%$ \\
\hline 551-3-L-400 & $\mathrm{L}$ & 400 & 487 & 4 & 564 & 5 & 4.9 & $1.0 \%$ \\
\hline 551-3-T-400 & $\mathrm{T}$ & 400 & 497 & 6 & 574 & 4 & 5.8 & $0.5 \%$ \\
\hline $551-4-L-400$ & L & 400 & 616 & 5 & 715 & 4 & 2.6 & $0.2 \%$ \\
\hline $551-5-L-400$ & $\mathrm{~L}$ & 400 & 494 & 2 & 610 & 4 & 9.3 & $0.9 \%$ \\
\hline $551-2-2-L-450$ & $\mathrm{~L}$ & 450 & 520 & 19 & 606 & 7 & 9.0 & $0.6 \%$ \\
\hline 551-2-2-L-550 & $\mathrm{L}$ & 550 & 137 & 2 & 169 & 5 & 73.3 & $7.2 \%$ \\
\hline 551-2-2-T-550 & $\mathrm{T}$ & 550 & 99 & 13 & 144 & 25 & 42.2 & $6.3 \%$ \\
\hline 551-3-L-550 & L & 550 & 165 & 6 & 200 & 2 & 69.1 & $6.5 \%$ \\
\hline 551-3-T-550 & $\mathrm{T}$ & 550 & 162 & 2 & 197 & 5 & 65.6 & $2.4 \%$ \\
\hline 551-4-L-550 & L & 550 & 152 & 6 & 193 & 6 & 45.9 & $3.3 \%$ \\
\hline 551-4-T-550 & $\mathrm{T}$ & 550 & 153 & 4 & 192 & 8 & 33.7 & $7.2 \%$ \\
\hline 551-5-L-550 & L & 550 & 229 & 2 & 269 & 2 & 39.7 & $3.7 \%$ \\
\hline 551-5-T-550 & $\mathrm{T}$ & 550 & 199 & 7 & 274 & 5 & 46.2 & $0.9 \%$ \\
\hline
\end{tabular}


TEM-10300-1

$03 / 01 / 2012$

TECHNICAL EVALUATION

Page 29 of 38

Rev. 03

Title: $\quad$ Elevated Temperature Tensile Tests on DU-10Mo Rolled Foils

$\begin{array}{lllll}\text { TEV No.: } & 2194 & \text { Rev. No.: } 2 & \text { Project No.: } 25228 & \text { Date: } 05 / 02 / 18\end{array}$

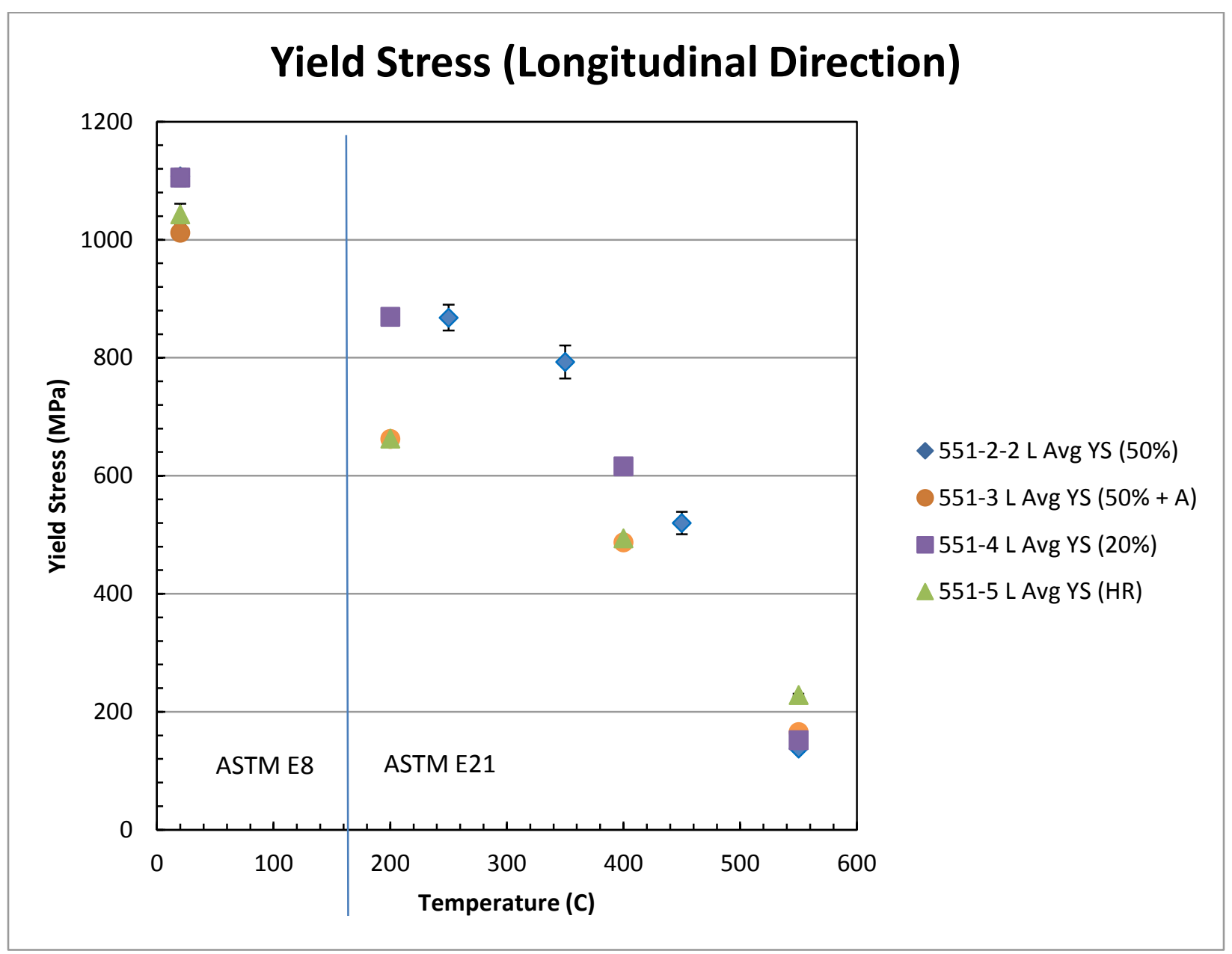

Figure 11. Yield stress in the longitudinal direction for each of the four foil fabrication conditions. The figure shows the cluster of yield stress at room temperature and at $550^{\circ} \mathrm{C}$ while there is a divergence in the fabrication conditions in the intermediate values. The annealed and hot-rolled-only foils indicate lower yield stress values in the intermediate temperatures. 
TEM-10300-1

$03 / 01 / 2012$

TECHNICAL EVALUATION

Page 30 of 38

Rev. 03

Title: $\quad$ Elevated Temperature Tensile Tests on DU-10Mo Rolled Foils

$\begin{array}{lllll}\text { TEV No.: } 2194 & \text { Rev. No.: } 2 & \text { Project No.: } 25228 & \text { Date: } 05 / 02 / 18\end{array}$

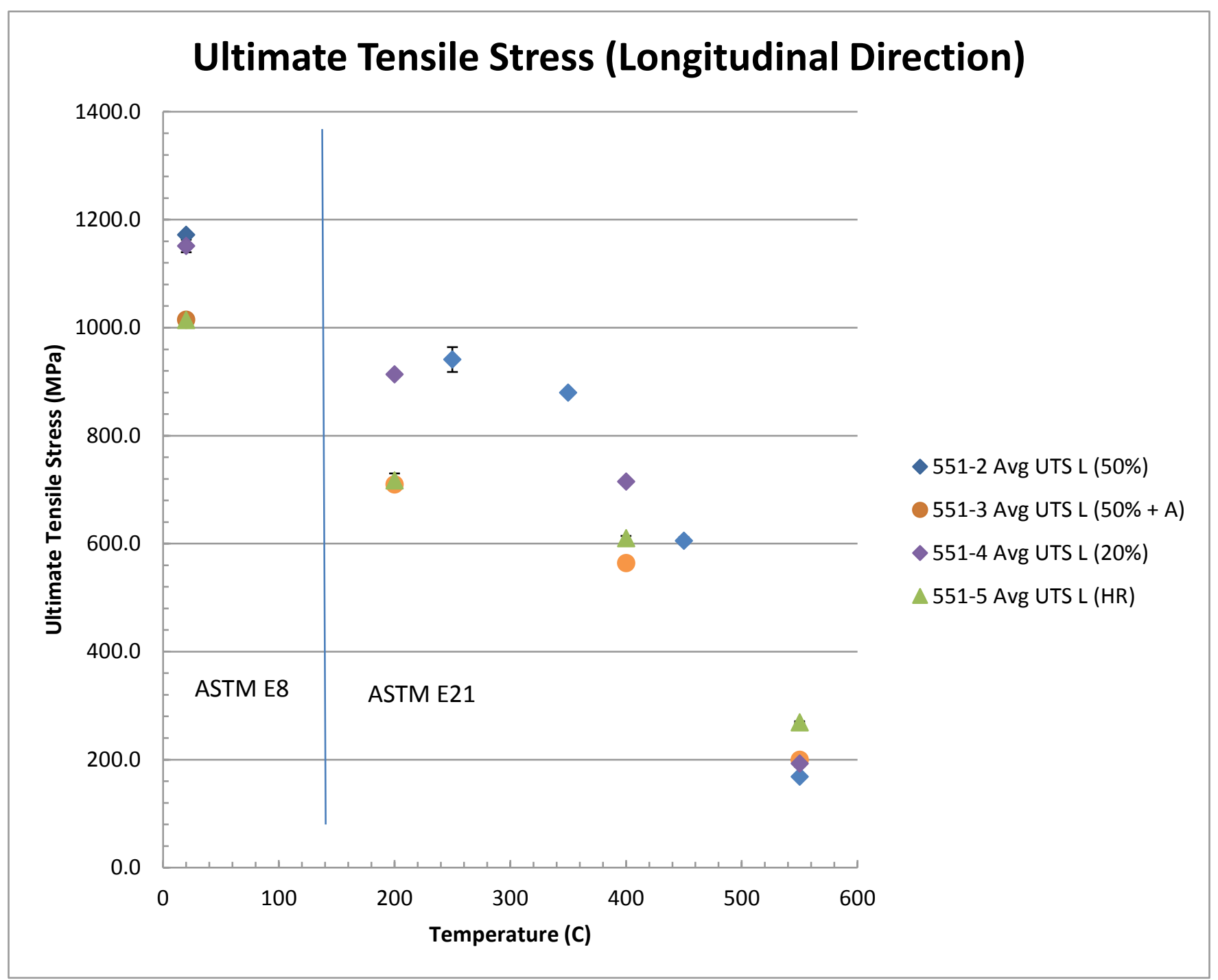

Figure 12. Ultimate tensile stress in the longitudinal direction for each of the four foil-fabrication conditions. The figure shows the cluster of ultimate tensile stress at $550^{\circ} \mathrm{C}$ while there is a divergence in the fabrication conditions in the room temperature and intermediate values. The annealed and hotrolled-only foils indicate lower ultimate tensile stress values in the room and intermediate temperatures. 
TEM-10300-1

$03 / 01 / 2012$

TECHNICAL EVALUATION

Page 31 of 38

Rev. 03

Title: $\quad$ Elevated Temperature Tensile Tests on DU-10Mo Rolled Foils

$\begin{array}{lllll}\text { TEV No.: } 2194 & \text { Rev. No.: } 2 & \text { Project No.: } 25228 & \text { Date: } 05 / 02 / 18\end{array}$

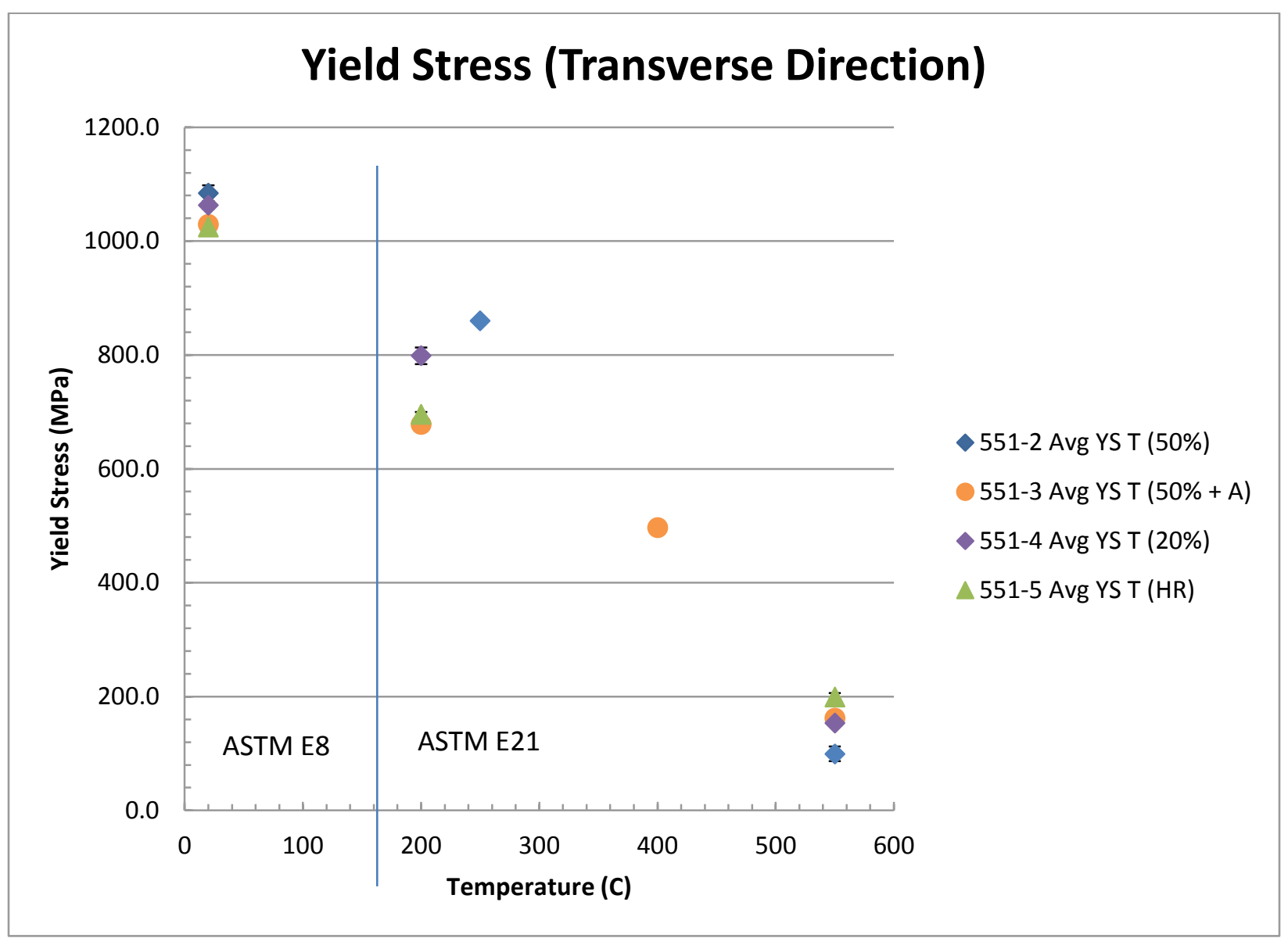

Figure 13. Yield stress in the transverse direction for each of the four foil-fabrication conditions. The figure shows the cluster of yield stress at room temperature and at $550^{\circ} \mathrm{C}$ while there is a divergence in the fabrication conditions in the intermediate values. The annealed and hot-rolled only foils indicate lower yield stress values in the intermediate temperatures. Note that data are not available for foils 5514 and $551-5$ in the transverse direction at $400^{\circ} \mathrm{C}$. 
TEM-10300-1

$03 / 01 / 2012$

TECHNICAL EVALUATION

Page 32 of 38

Rev. 03

Title: $\quad$ Elevated Temperature Tensile Tests on DU-10Mo Rolled Foils

$\begin{array}{lllll}\text { TEV No.: } 2194 & \text { Rev. No.: } 2 & \text { Project No.: } 25228 & \text { Date: } 05 / 02 / 18\end{array}$

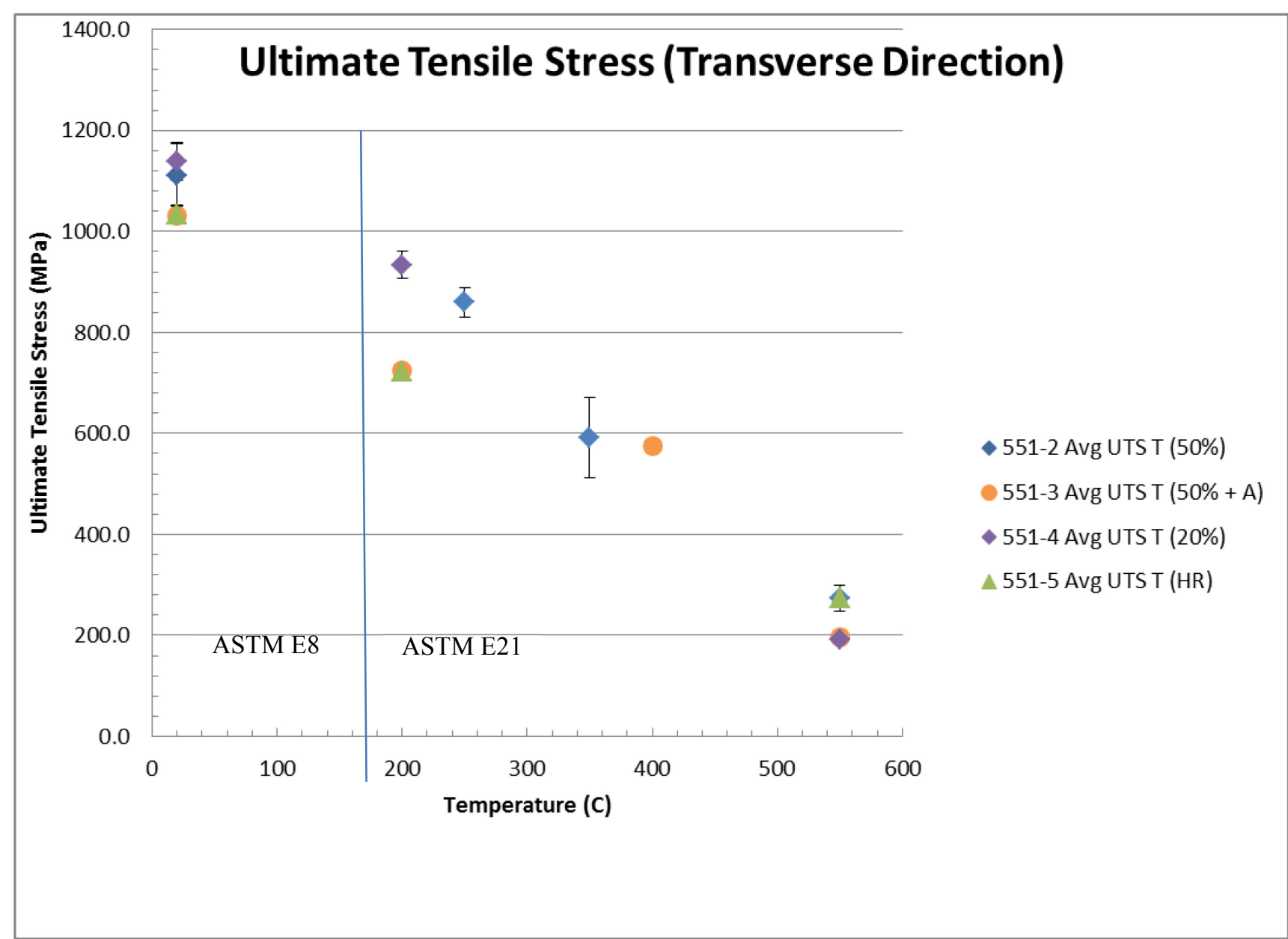

Figure 14. Ultimate tensile stress in the transverse direction for each of the four foil-fabrication conditions. The figure shows the cluster of yield stress at room temperature and at $550^{\circ} \mathrm{C}$ while there is a divergence in the fabrication conditions in the intermediate values. The annealed and hot-rolled-only foils indicate lower yield stress values in the intermediate temperatures $\left(200^{\circ} \mathrm{C}\right)$, but this seems to reconverge at $400^{\circ} \mathrm{C}$. Note that data are not available for foils $551-4$ and $551-5$ in the transverse direction at $400^{\circ} \mathrm{C}$. 
TEM-10300-1

$03 / 01 / 2012$

TECHNICAL EVALUATION

Page 33 of 38

Rev. 03

Title: $\quad$ Elevated Temperature Tensile Tests on DU-10Mo Rolled Foils

$\begin{array}{lllll}\text { TEV No.: } & 2194 & \text { Rev. No.: } 2 & \text { Project No.: } 25228 & \text { Date: } 05 / 02 / 18\end{array}$

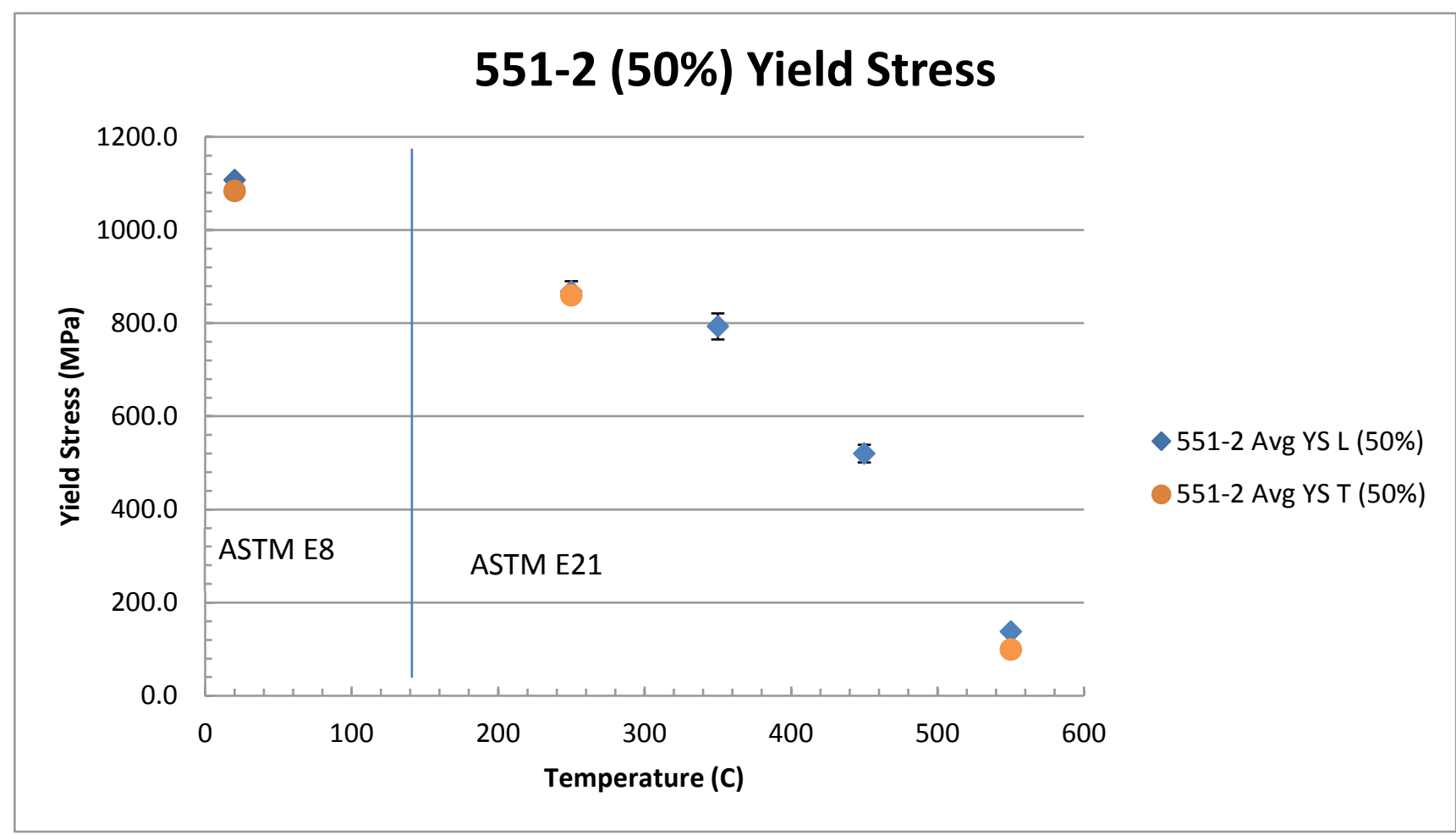

Figure 15. Yield stress for foils cold-rolled to $50 \%$ reduction. Note no significant difference in yield stress values based on rolling direction. No data are available for yield stress in the transvers direction for 350 and $450^{\circ} \mathrm{C}$.

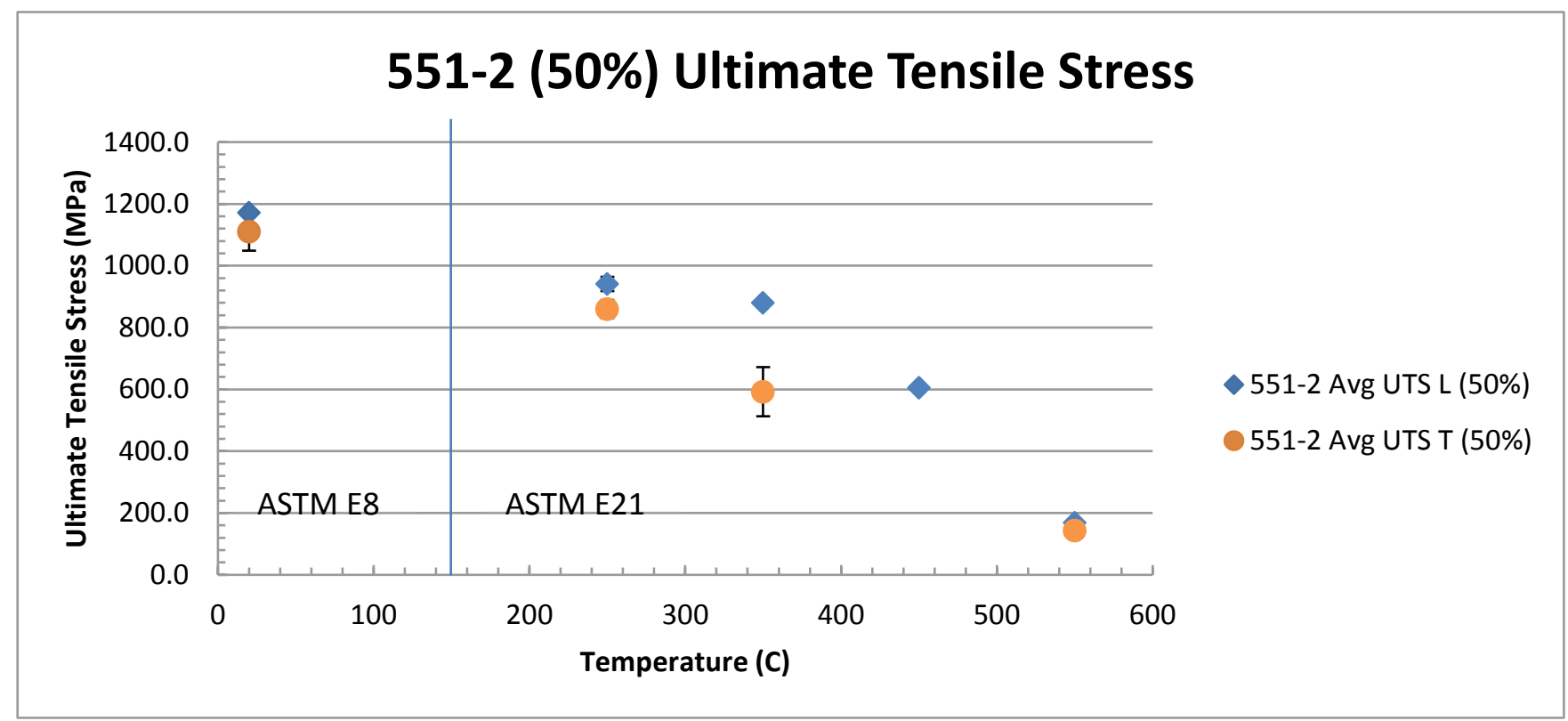

Figure 16. Ultimate tensile stress for foils cold-rolled to $50 \%$ reduction and then annealed at $650^{\circ} \mathrm{C}$ for one hour. Note no significant difference in ultimate tensile stress values based on rolling direction. 
TEM-10300-1

$03 / 01 / 2012$

TECHNICAL EVALUATION

Page 34 of 38

Rev. 03

Title: $\quad$ Elevated Temperature Tensile Tests on DU-10Mo Rolled Foils

\begin{tabular}{llllll} 
TEV No.: & 2194 & Rev. No.: 2 & Project No.: & 25228 & Date: \\
\hline
\end{tabular}

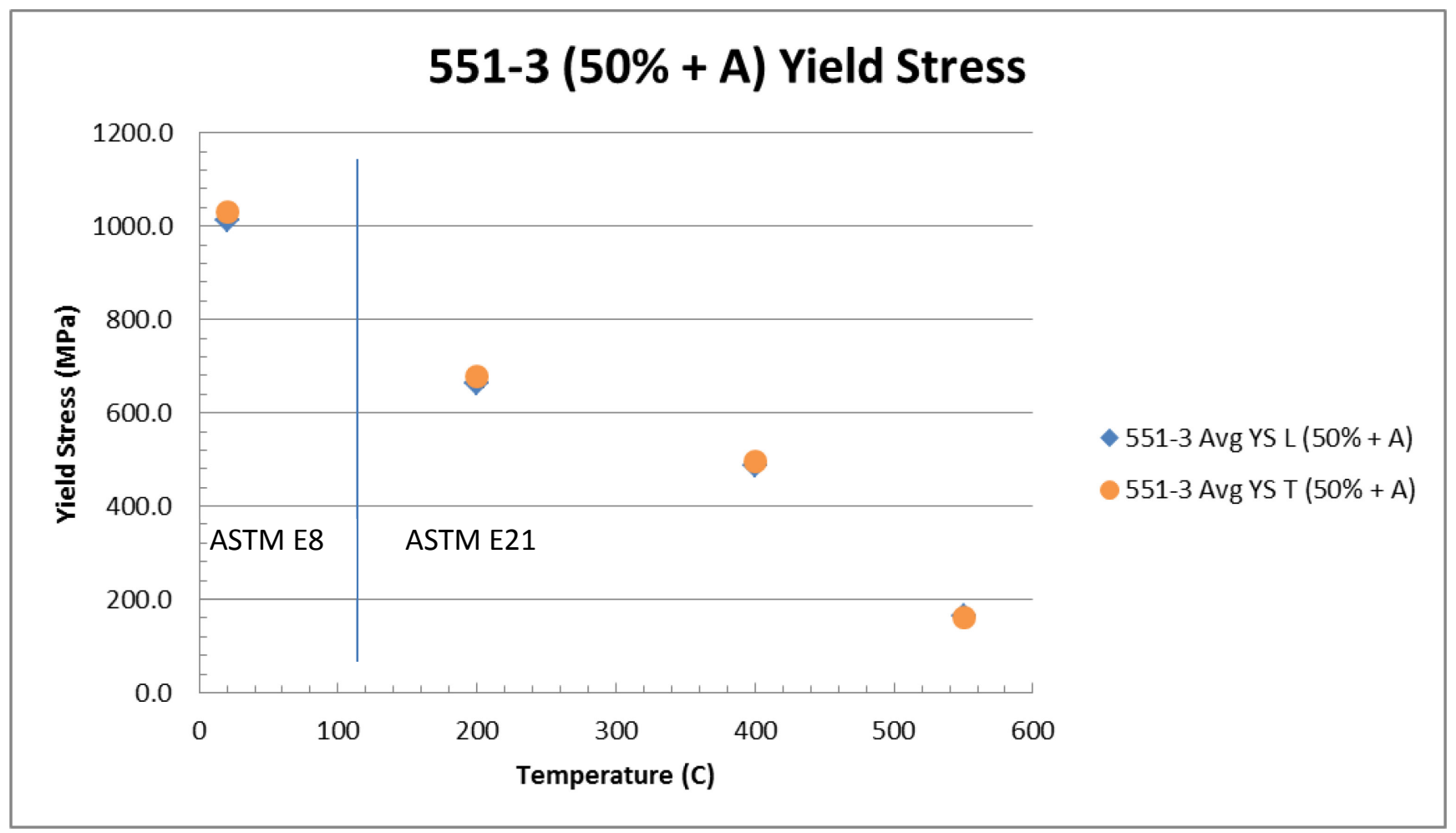

Figure 17 . Yield stress for foils cold-rolled to $50 \%$ reduction and then annealed at $650^{\circ} \mathrm{C}$ for one hour. Note no significant difference in yield stress values based on rolling direction.

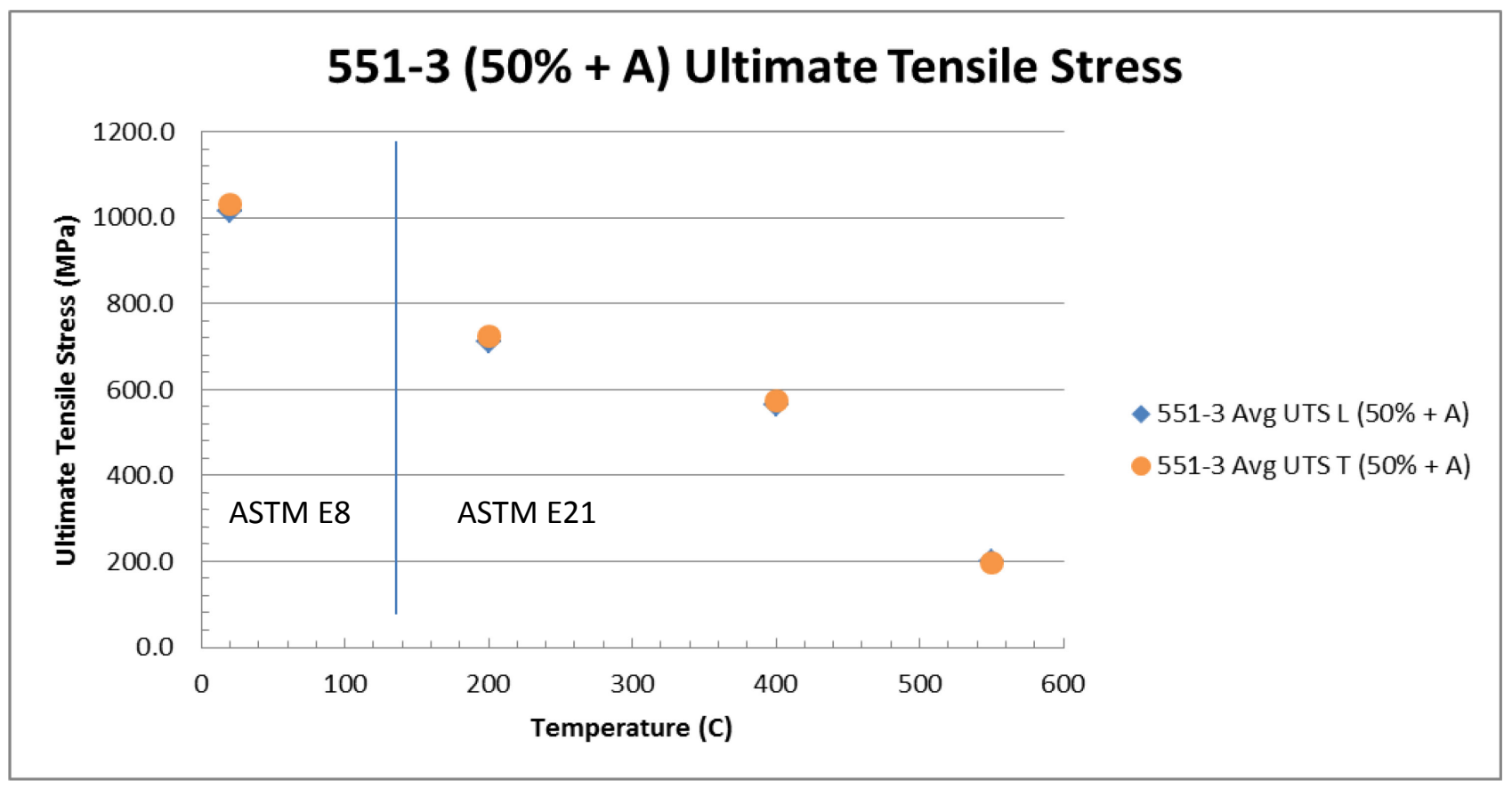

Figure 18. Ultimate tensile stress for foils cold-rolled to $50 \%$ reduction and then annealed at $650^{\circ} \mathrm{C}$ for one hour. Note no significant difference in ultimate tensile stress values based on rolling direction. 
TEM-10300-1

$03 / 01 / 2012$

TECHNICAL EVALUATION

Page 35 of 38

Rev. 03

Title: $\quad$ Elevated Temperature Tensile Tests on DU-10Mo Rolled Foils

\begin{tabular}{llllll} 
TEV No.: & 2194 & Rev. No.: 2 & Project No.: & 25228 & Date: \\
\hline
\end{tabular}

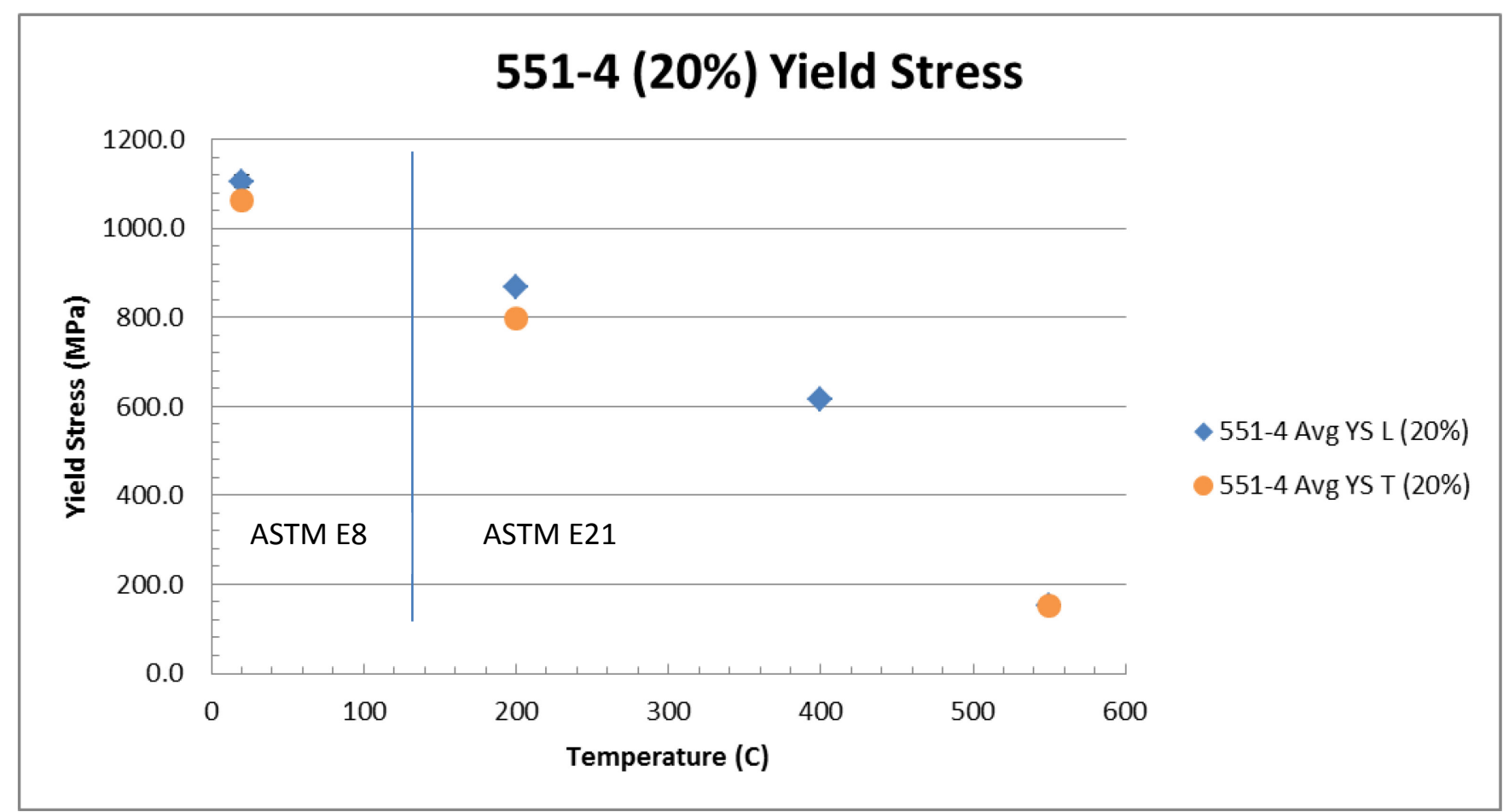

Figure 19. Yield stress for foils cold-rolled to $20 \%$ reduction. Note a minor difference in yield stress values based on rolling direction for $200^{\circ} \mathrm{C}$. No transverse data are available for $400^{\circ} \mathrm{C}$.

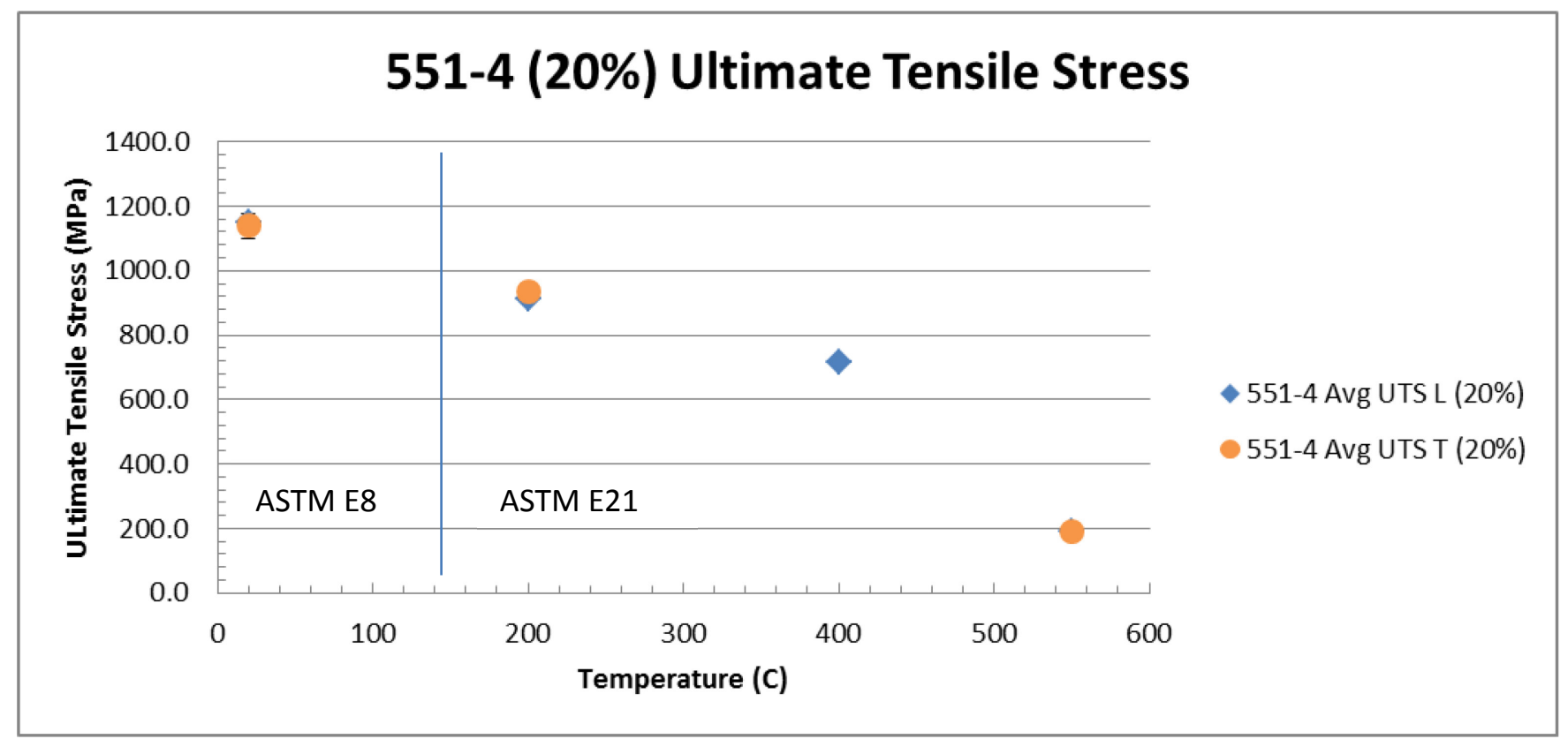

Figure 20. Ultimate tensile stress for foils hot-rolled only with no cold-rolling reduction. Note no significant difference in ultimate tensile-stress-values-based temperature. No transverse data are available for $400^{\circ} \mathrm{C}$. 
TEM-10300-1

$03 / 01 / 2012$

TECHNICAL EVALUATION

Page 36 of 38

Rev. 03

Title: $\quad$ Elevated Temperature Tensile Tests on DU-10Mo Rolled Foils

\begin{tabular}{llllll} 
TEV No.: & 2194 & Rev. No.: 2 & Project No.: & 25228 & Date: \\
\hline
\end{tabular}

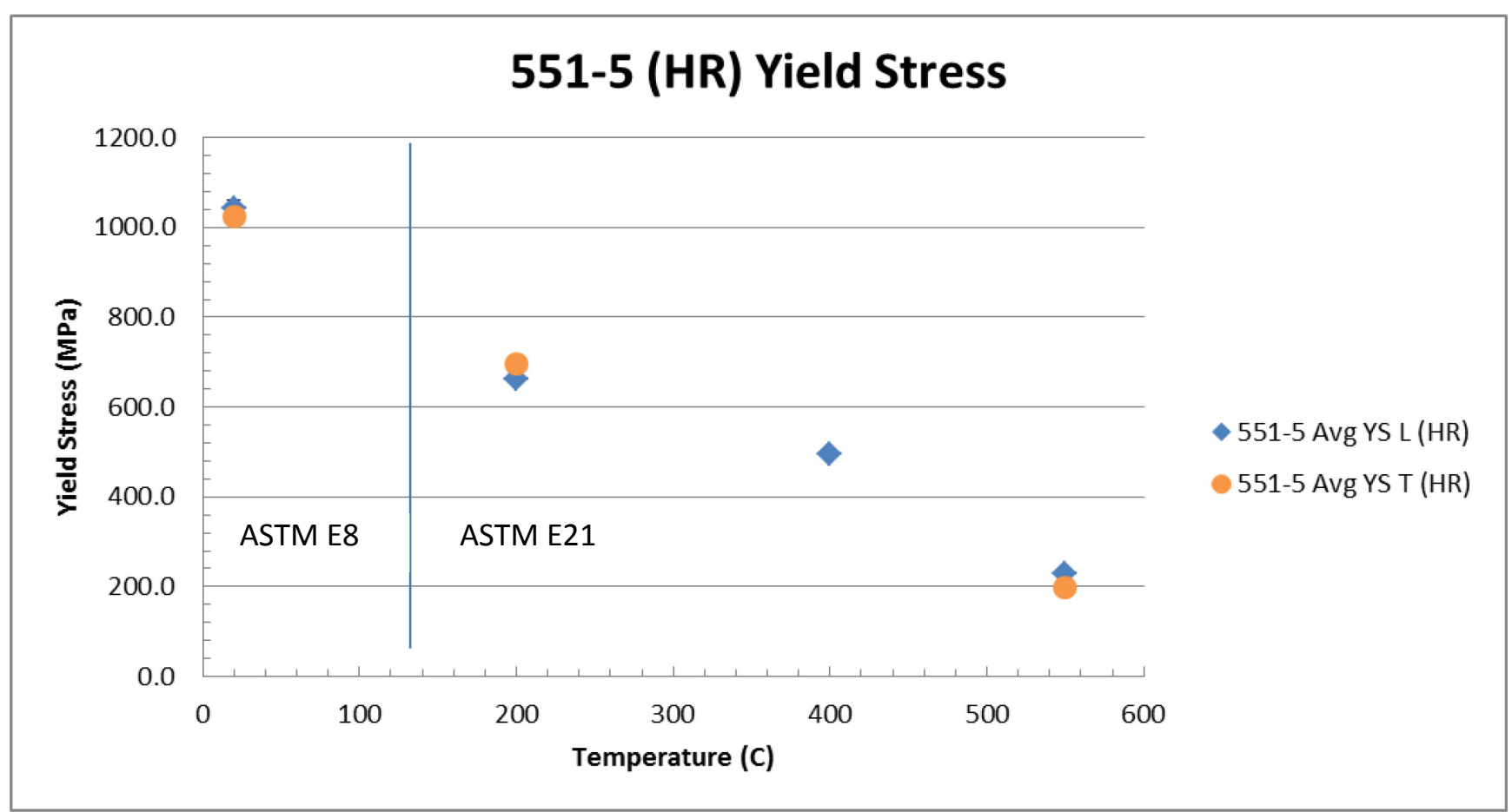

Figure 21. Yield stress for foils hot-rolled only with no cold-rolling reduction. Note no significant difference in yield stress values based temperature. No transverse data available for $400^{\circ} \mathrm{C}$.

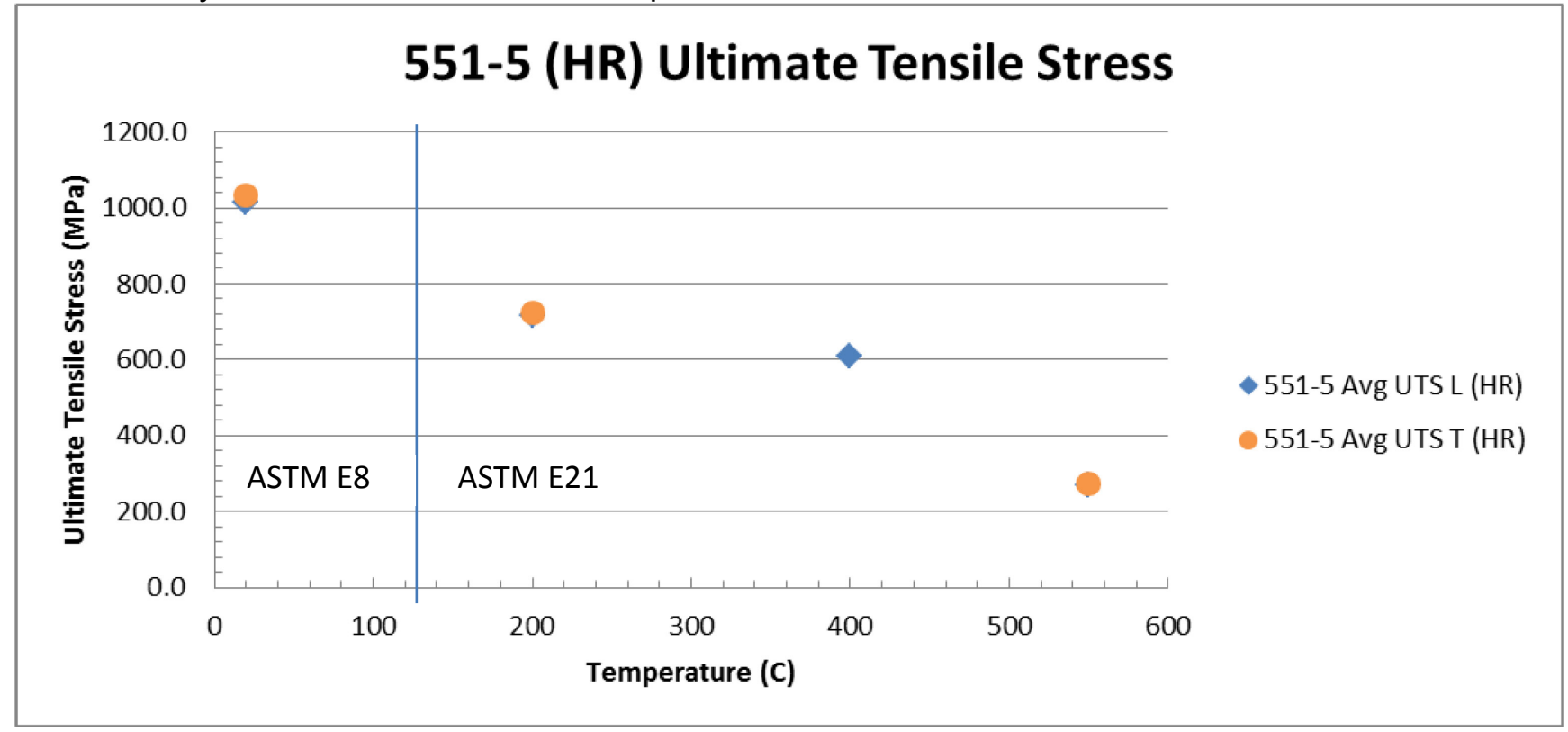

Figure 22. Ultimate tensile stress for foils hot-rolled only with no cold-rolling reduction. Note no significant difference in ultimate tensile stress values based temperature. No transverse data available for $400^{\circ} \mathrm{C}$. 
Rev. 03

Title: $\quad$ Elevated Temperature Tensile Tests on DU-10Mo Rolled Foils

$\begin{array}{llllll}\text { TEV No.: } 2194 & \text { Rev. No.: } 2 & \text { Project No.: } 25228 & \text { Date: } 05 / 02 / 18\end{array}$

\section{REFERENCES}

1. PLN-4560, "GTRI Mechanical Property Characterization Test Plan"

2. J. E. Gates, et al., "Stress-Strain Properties of Irradiated Uranium-10 w/o Molybdenum," BMI-APDA-638, Battelle Memorial Institute, Columbus, OH, January, 1958.

3. G. Beghi, "Gamma Phase Uranium-Molybdenum Fuel Alloys”, EUR-4053e, European Atomic Energy Community, 1968.

4. M.B. Waldron, R.C. Burnett, S.F. Pugh, "The Mechanical Properties of Uranium-Molybdenum Alloys," UK Atomic Energy Authority Technical Report, ARE-MB-2554, 1958.

5. Hakan Ozaltun, M-H., Herman Shen, and Pavel Medvedev, "Assessment of residual stresses on U10Mo alloy based monolithic mini-plates during Hot Isostatic Pressing," Journal of Nuclear Materials 419.1 (2011), 76-84.

6. D. Burkes at el., Metall. and Materials Trans. A 40A (2009), 1069-1079.

7. TEV-1921, "Tensile Testing System Configuration and Validation for DU-10Mo Elevated Temperature Tensile Tests," Rev. 0, INL, 2013.

8. LAB-2176, laboratory notebook.

9. Standard Practice for Verification and Classification of Extensometer Systems, ASTM E83-10, ASTM International Annual Book of Standards, v.3.01, 2013.

10. Standard Test Methods for Tension Testing of Metallic Materials, ASTM E8/8M-13a, ASTM International Annual Book of Standards, v.3.01, 2013.

11. Standard test Methods for Elevated Temperature Tension Tests of Metallic Materials, ASTM E21-09, ASTM International Annual Book of Standards, v.3.01, 2013.

12. "Half-Subsize Flat Tensile Specimen Fabrication Specification (US Customary Dimensions)," INL Drawing 604240, 2013.

13. TEV-1920, "Preparation of DU-10Mo Foils for Elevated Temperature Tensile Tests," INL, 2013.

14. J. E. Gates, et al., "Stress-Strain Properties of Irradiated Uranium-10 w/o Molybdenum," BMI-APDA-638, Battelle Memorial Institute, Columbus, OH, January, 1958.

15. G. Beghi, "Gamma Phase Uranium-Molybdenum Fuel Alloys," EUR-4053e, European Atomic Energy Community, 1968.

16. D. Burkes at el., Metall. and Materials Trans. A 40A (2009), 1069-1079. 
Rev. 03

Title: $\quad$ Elevated Temperature Tensile Tests on DU-10Mo Rolled Foils

$\begin{array}{llllll}\text { TEV No.: } & 2194 & \text { Rev. No.: } 2 & \text { Project No.: } 25228 & \text { Date: } 05 / 02 / 18\end{array}$

17. Standard Test Method for Young's Modulus, Tangent Modulus, and Chord Modulus, ASTM E111-04, ASTM International Annual Book of Standards, v.3.01, 2013

18. J. E. Gates, et al., "Stress-Strain Properties of Irradiated Uranium-10 w/o Molybdenum," BMI-APDA-638, Battelle Memorial Institute, Columbus, OH, January, 1958.

19. G. Beghi, "Gamma Phase Uranium-Molybdenum Fuel Alloys," EUR-4053e, European Atomic Energy Community, 1968.

20. Standard Practice for Measuring Ultrasonic Velocity in Materials, ASTM E494-10, ASTM International Annual Book of Standards, v.3.01, 2013 
TEM-10300-1

$03 / 01 / 2012$

TECHNICAL EVALUATION

Page $\mathrm{A} 1$ of $\mathrm{A} 4$

Rev. 03

Title: $\quad$ Elevated Temperature Tensile Tests on DU-10Mo Rolled Foils

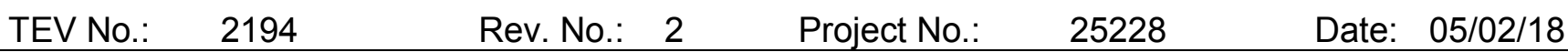

Appendix A

Additional Figures

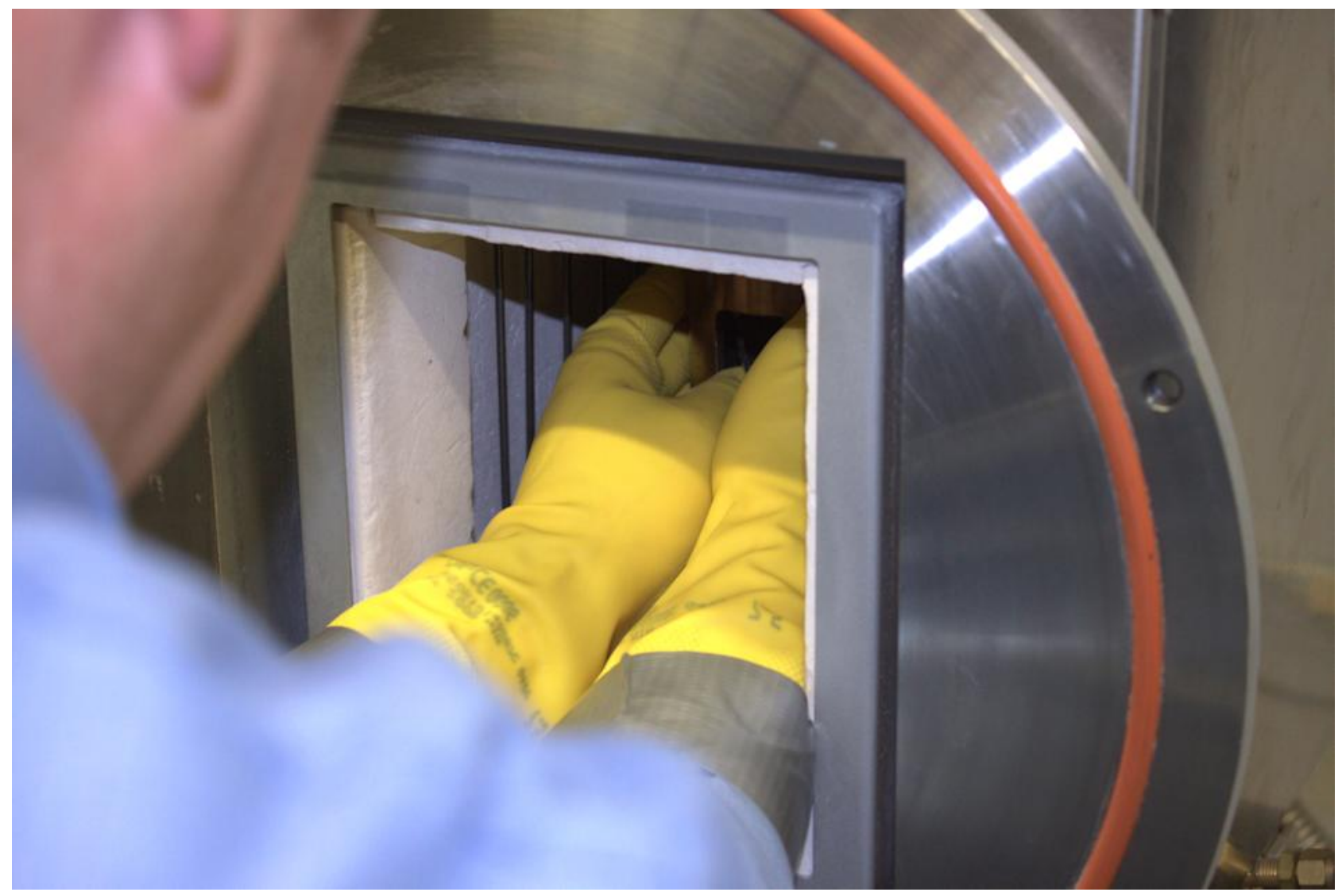

Figure 23. Minimal area inside furnace to install grip alignment collar, install specimen in grips, ensure proper specimen alignment in grips, tighten grip jaws, remove alignment collar, and install extensometer (when used). Care must be used to prevent damage to specimens or fragile Super Kanthal heating elements. 
TEM-10300-1

$03 / 01 / 2012$

TECHNICAL EVALUATION

Page $\mathrm{A} 2$ of $\mathrm{A} 4$

Rev. 03

Title: $\quad$ Elevated Temperature Tensile Tests on DU-10Mo Rolled Foils

\begin{tabular}{|c|c|c|c|}
\hline & Rev. No.: & Project $N$ \\
\hline
\end{tabular}

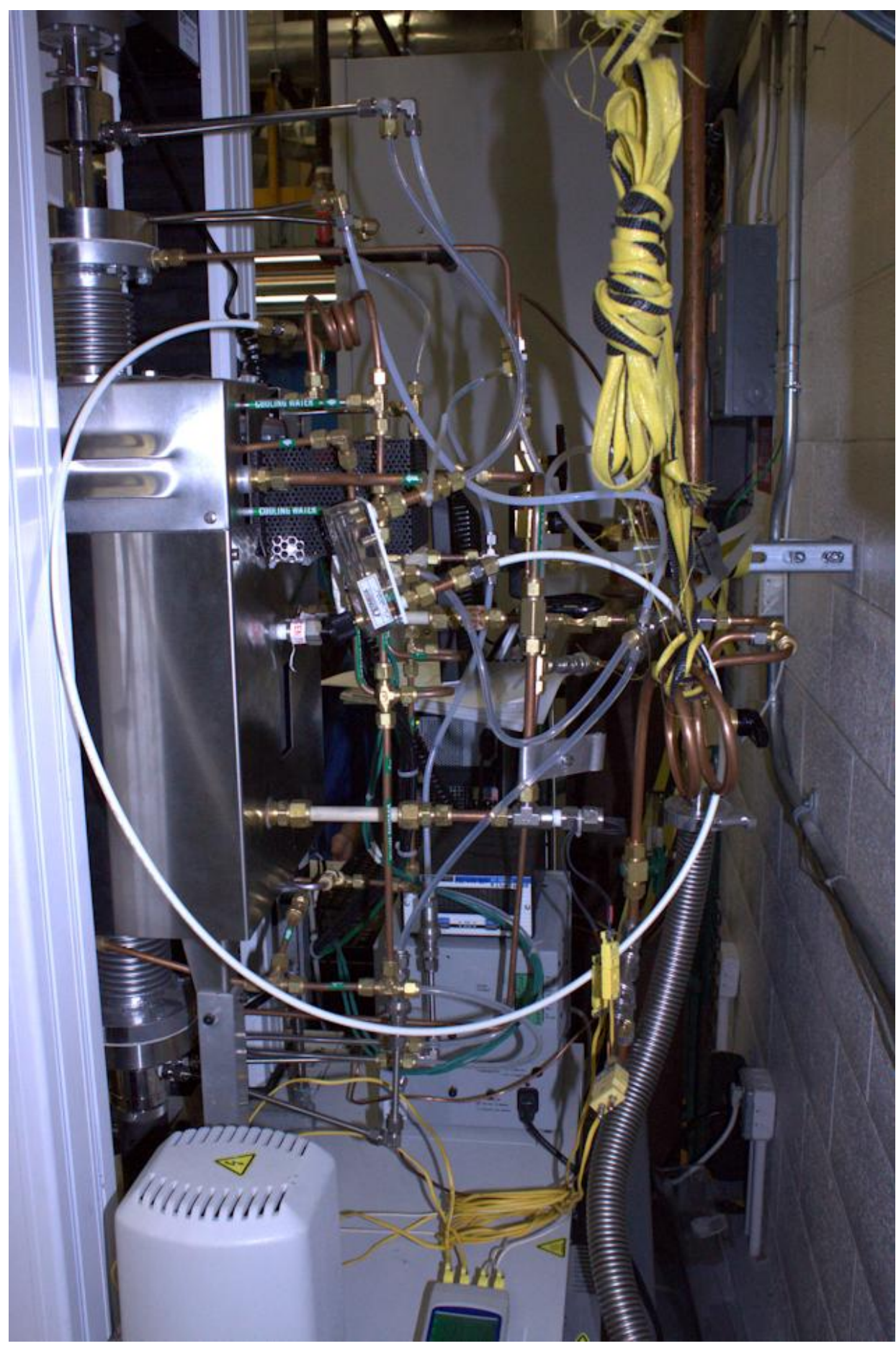

Figure 24. Inert-gas and cooling-water plumbing at rear of furnace. Metal vacuum hose running down to pump is seen at lower right. Gas piping is all metal or ceramic to prevent oxygen infusion. 
TEM-10300-1

$03 / 01 / 2012$

TECHNICAL EVALUATION

Page $\mathrm{A} 3$ of $\mathrm{A} 4$

Rev. 03

Title: $\quad$ Elevated Temperature Tensile Tests on DU-10Mo Rolled Foils

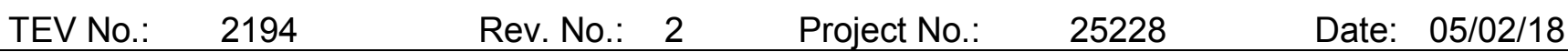

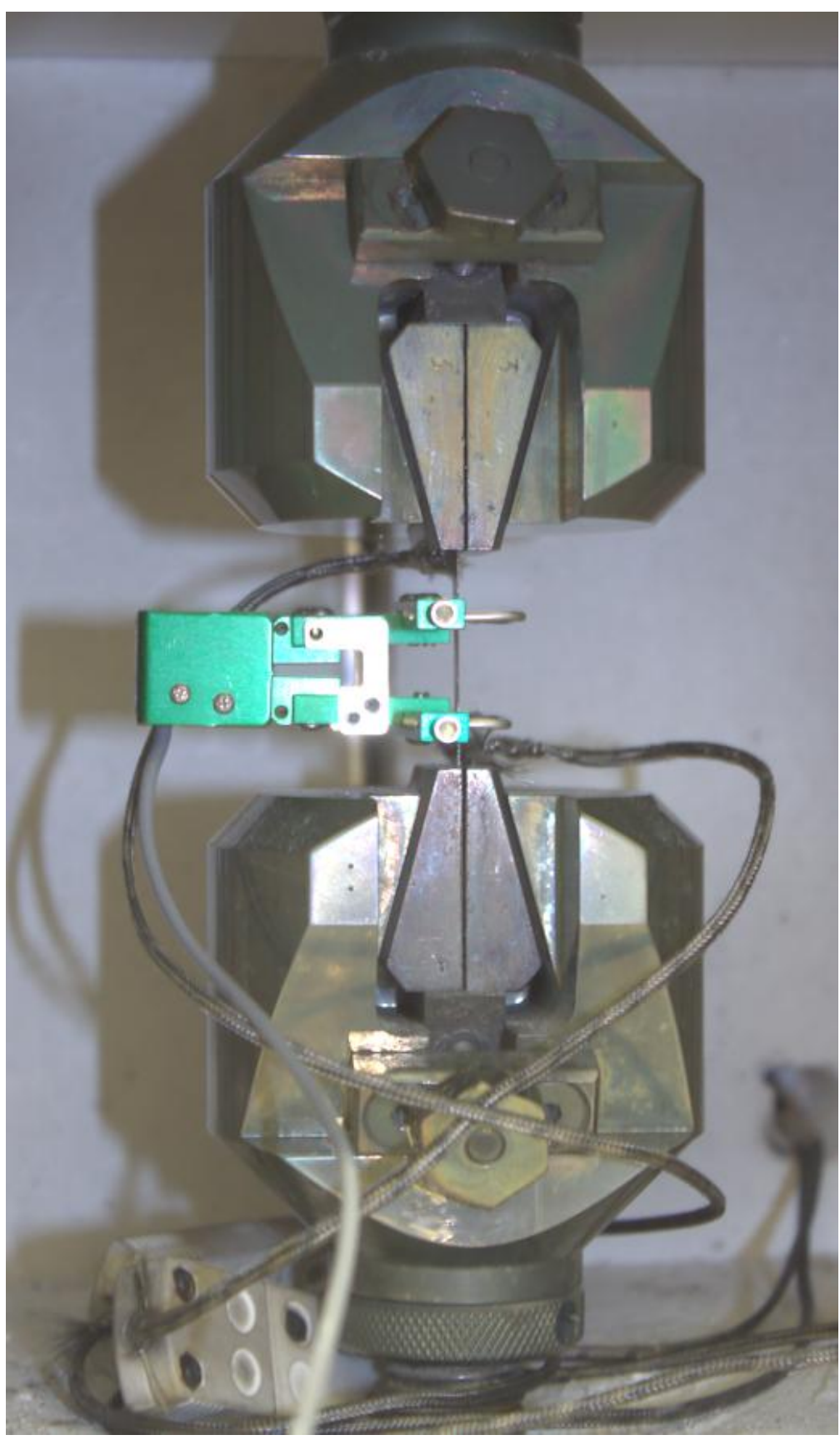

Figure 25. Specimen installed in grips inside of furnace chamber, with extensometer installed, ready to begin a room-temperature test. Wires from thermocouples welded to grip jaws are seen above and below extensometer. For size reference, the hex grip-tightening bolt-heads are $16 \mathrm{~mm}$. Grip alignment collar has been removed. 
TEM-10300-1

$03 / 01 / 2012$

Rev. 03

Title: $\quad$ Elevated Temperature Tensile Tests on DU-10Mo Rolled Foils

\begin{tabular}{|c|c|c|c|}
\hline & Rev. No.: & Project $N$ \\
\hline
\end{tabular}

Figure 26. Broken elevated-temperature test specimen as seen through magnifying viewport in furnace door. Lighting is through a narrow window in the door below the viewport. Break is visible at lower portion of reduced section.

Table 9. Chemistry report provided by $Y-12$ for log 3C32-WP-TRN0. Impurity levels over 25 ppm reported. Impurity values less than 25 ppm where provided in the chemistry report from $\mathrm{Y}-12$ but are not reported here.

\begin{tabular}{|l|l|l|l|l|l|l|l|l|l|l|l|l|}
\hline $\begin{array}{c}\text { 3C32- } \\
\text { WP- } \\
\text { TRNO }\end{array}$ & \%Mo & ppm C & $\begin{array}{c}\text { ppm } \\
\text { Al }\end{array}$ & $\begin{array}{c}\text { ppm } \\
\text { Cu }\end{array}$ & $\begin{array}{c}\text { ppm } \\
\text { Er }\end{array}$ & $\begin{array}{c}\text { ppm } \\
\text { Fe }\end{array}$ & ppm K & $\begin{array}{c}\text { ppm } \\
\text { Mn }\end{array}$ & $\begin{array}{c}\text { Ppm } \\
\text { Ni }\end{array}$ & ppm P & $\begin{array}{c}\text { ppm } \\
\text { Si }\end{array}$ & $\begin{array}{c}\text { ppm } \\
\text { W }\end{array}$ \\
\hline Top & 10.40 & 706 & 60 & 13 & 5.9 & 160 & 32 & 28 & 37 & $<20$ & 250 & 28 \\
\hline Middle & 10.50 & 714 & 60 & 13 & 28 & 160 & $<16$ & 29 & 39 & $<20$ & 250 & 25 \\
\hline Bottom & 10.30 & 722 & 61 & 12 & 3.0 & 160 & 21 & 29 & 38 & $<20$ & 240 & 25 \\
\hline
\end{tabular}


Title:

\section{Appendix B}

\section{Tensile Testing Dimensional Measurements}

Date: $9-12-13$

Measurement Operator

$\mathrm{GL}$ measurement instrument

$W$ measurement instrument

T measurement instrument

Temperature instrument
1. Locations to measure

1. Locations to measure are indicated in the diagram. All dimensions in $\mathrm{mm}$. Record measurements to $0.001 \mathrm{~mm}$.

. Conical indent marks for $\mathrm{GL}$ are small, less than $0.1 \mathrm{~mm}$ typical. Measure indents center-to-center three times with optical comparator. Reset reference zero for

each measurement (three independent measurements).

3. Measure $W_{1}, W_{2}$, and $W_{c}$ two times. Reset reference zero for each measurement (two independent measurements).

4. Measure two thicknesses at each of two locations inside of gage mark indents as indicated. Measuring device contact surface should not cover the indent. Ball tip 5. Record room temperature

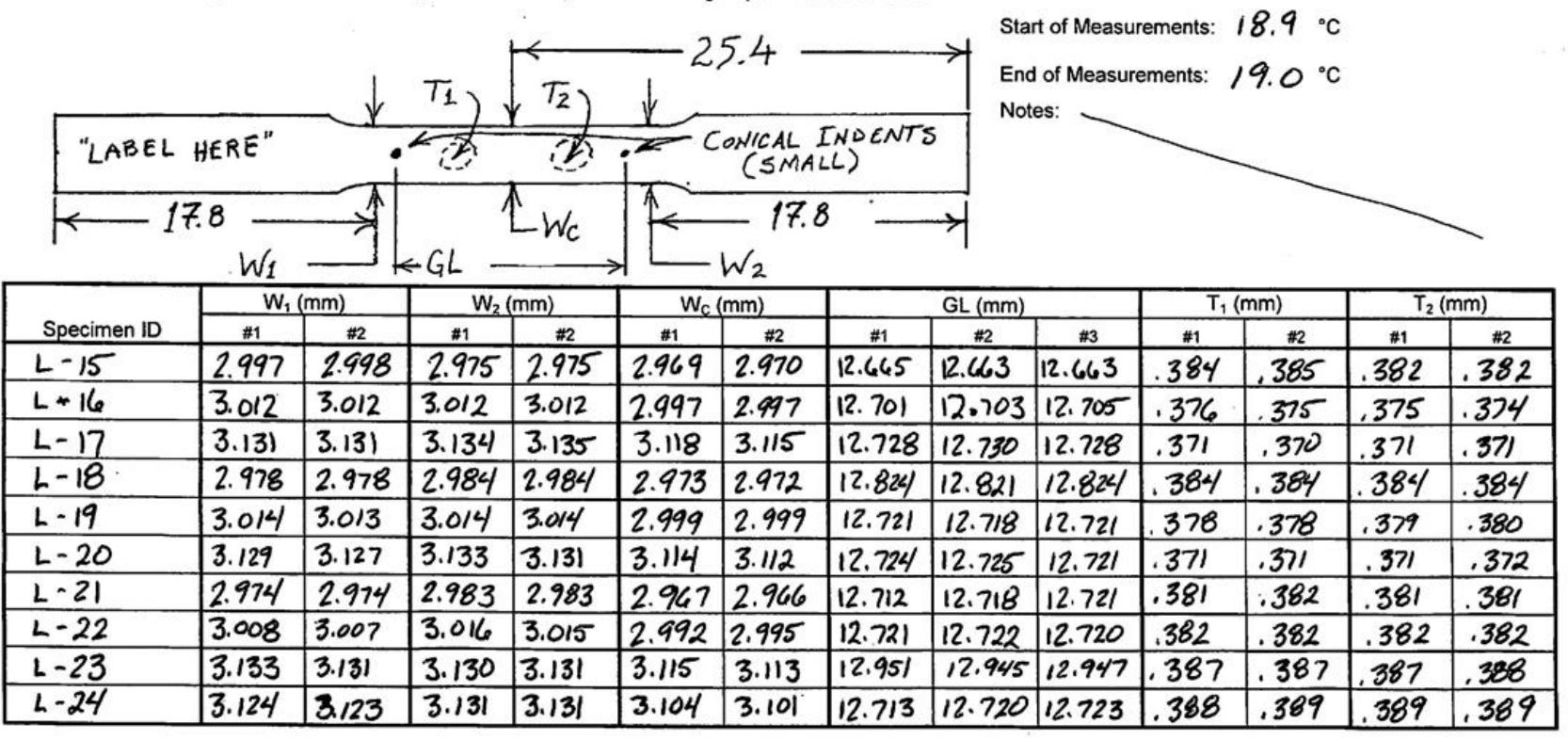


Rev. 03

Elevated Temperature Tensile Tests on DU-10Mo Rolled Foils

Title:

2194

Rev. No.:

2

Project No.:

25228

Date:

05/02/18

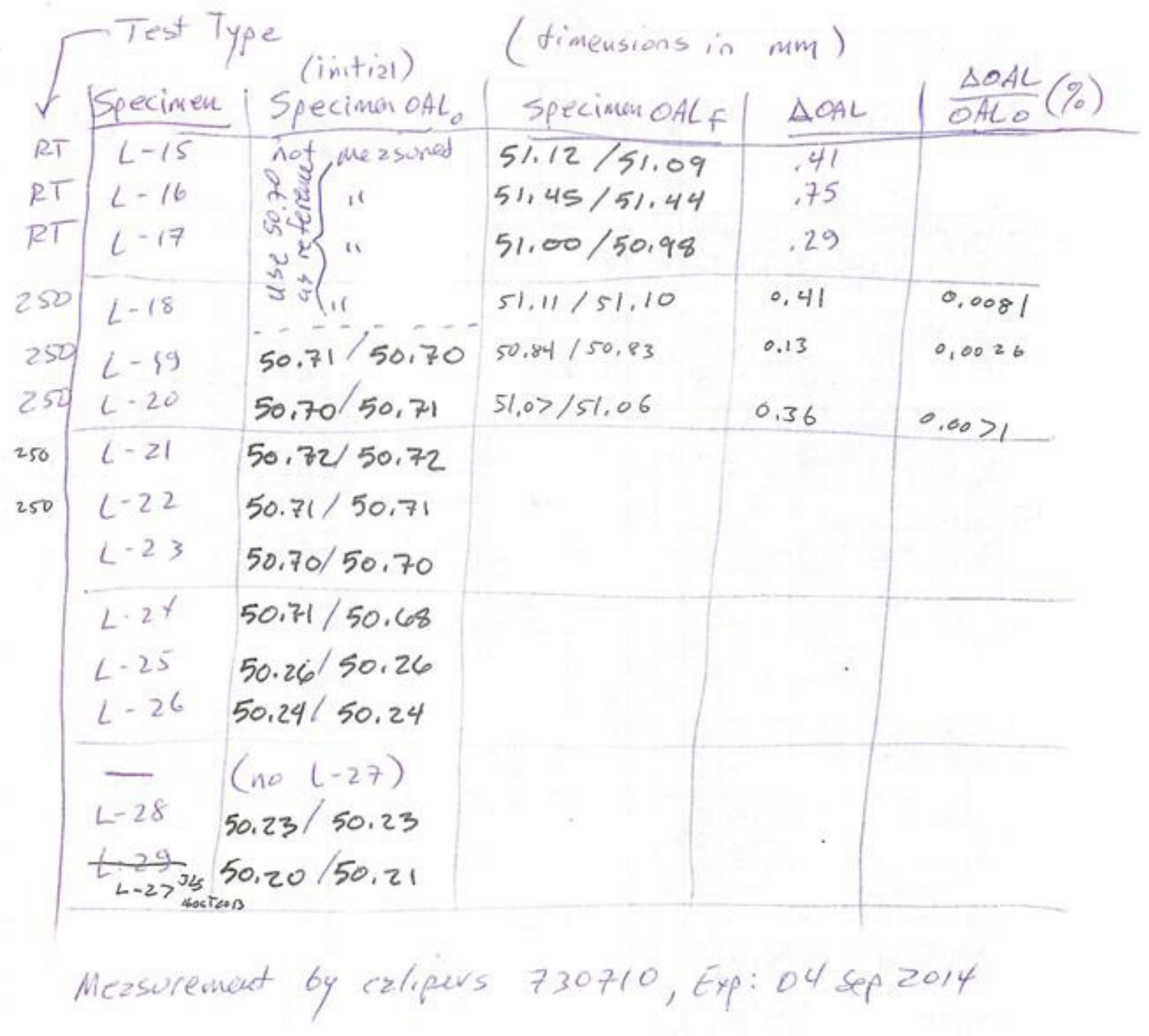


Rev. 03

\section{Elevated Temperature Tensile Tests on DU-10Mo Rolled Foils}

Title:

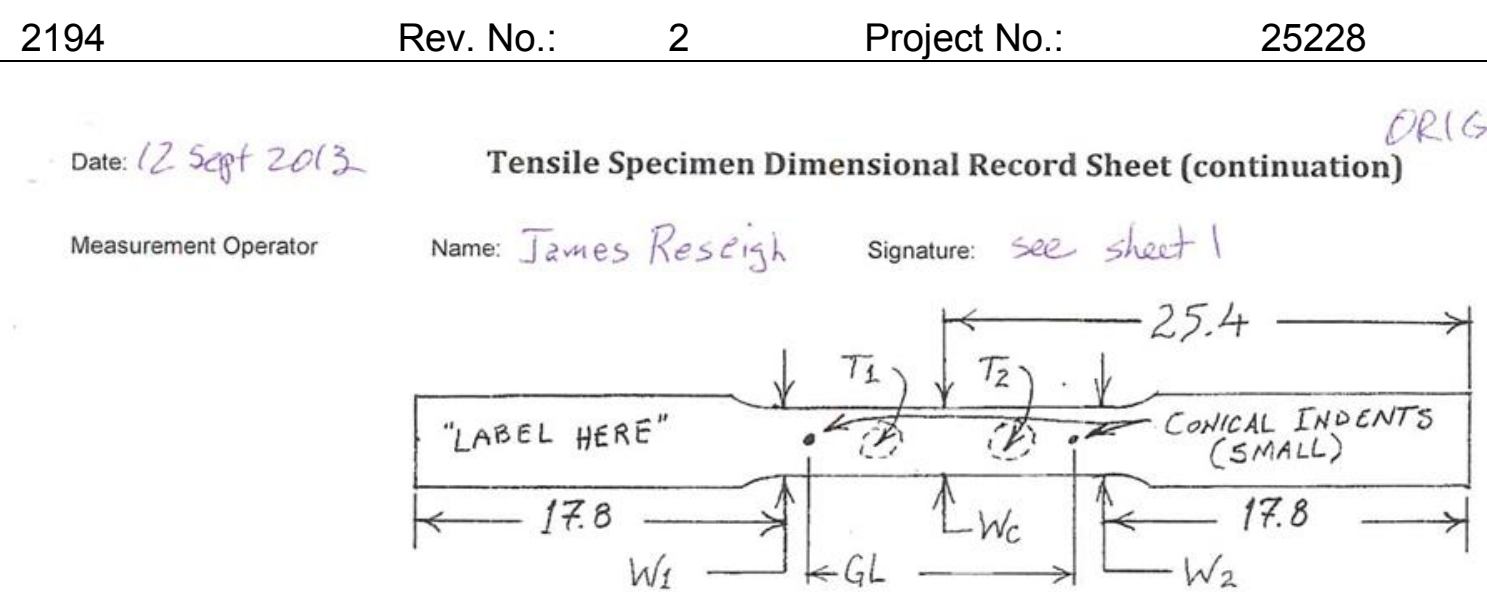

\begin{tabular}{|c|c|c|c|c|c|c|c|c|c|c|c|c|c|}
\hline \multirow[b]{2}{*}{ Specimen ID } & \multicolumn{2}{|c|}{$W_{1}(\mathrm{~mm})$} & \multicolumn{2}{|c|}{$W_{2}(\mathrm{~mm})$} & \multicolumn{2}{|c|}{$W_{c}(\mathrm{~mm})$} & \multicolumn{3}{|c|}{$\mathrm{GL}(\mathrm{mm})$} & \multicolumn{2}{|c|}{$\mathrm{T}_{1}(\mathrm{~mm})$} & \multicolumn{2}{|c|}{$T_{2}(\mathrm{~mm})$} \\
\hline & $\# 1$ & $\# 2$ & $\# 1$ & $\# 2$ & $\# 1$ & $\# 2$ & $\# 1$ & $\# 2$ & $\# 3$ & $\# 1$ & $\# 2$ & $\# 1$ & $\# 2$ \\
\hline$L-25$ & 2.982 & 2.980 & 2.977 & 2.976 & 2.962 & 2.940 & 12.738 & 12.7410 & 12.748 & .373 & .374 & .373 & 373 \\
\hline$L-26$ & 3.008 & 3.008 & 3.014 & 3.013 & 2.998 & 2.996 & 12.699 & 12.684 & 12.678 & .381 & .381 & .381 & .381 \\
\hline & & & & & & & & & & .385 & 386 & 387 & .387 \\
\hline$L-28$ & 3.131 & 3.131 & 3.128 & 3.130 & 3.116 & 3.114 & 12.837 & 12.831 & 12.834 & .385 & 386 & .387 & .387 \\
\hline$t-29\langle-2\rangle^{\frac{526}{160 r_{3}}}$ & 3.121 & 3.118 & 3.132 & 3.131 & 3.105 & 3.102 & 12.723 & 12.722 & 12.725 & .389 & 389 & .389 & .390 \\
\hline & & & & & & & & & & & & & \\
\hline & & & & & & & & & & & & & \\
\hline & & & & & & & & & & & & & \\
\hline & & & & & & & & & & & & & \\
\hline & & & & & & & & & & & & & \\
\hline & & & & & & & & & & & & & \\
\hline & & & & & & & & & & & & & \\
\hline & & & & & & & & & & & & & \\
\hline & & & & & & & & & & & & & \\
\hline
\end{tabular}


Rev. 03

Title:

TEV No.: 2194

Rev. No.:

2

Project No:

25228

Date:

$05 / 02 / 18$

Fo:l: 551-2-2 Pretest New

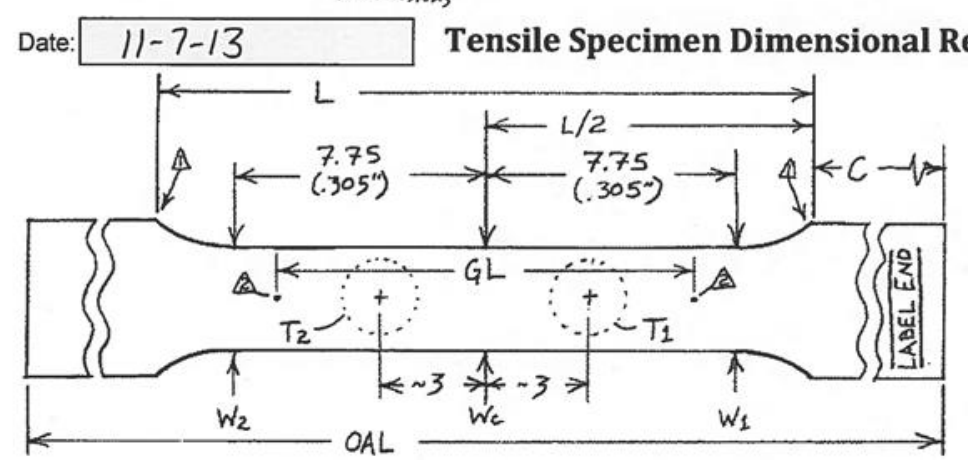

OAL measurement instrument ID: $A G 78$

GL measurement instrument ID: $A G 78$

W measurement instrument ID: $A$ G78

T measurement instrument ID: $A 3180$

Temperature instrument ID: 730725

Start of Measurements: $19.6{ }^{\circ} \mathrm{C}$

Sheet 1 of 3

Delta 1. Reference positions for reduced section locations - corner at intersection of end tab and fillet. Locate center between corners (L/2) as zero position reference. It is not necessary to record dimension L.

Delta 2. Conical indent marks for GL measurement are small, less than $0.1 \mathrm{~mm}$ typical. Measure indents center-to-center three times with optical comparator. Reset

reference zero for each measurement (three independent measurements).

1. Locations to measure are indicated in the diagram. All dimensions in mm except as indicated by (in.). Record measurements to $0.001 \mathrm{~mm}$ excepting " $\mathrm{C}$ " and "OAL."

2. Record dimension " $\mathrm{C}$ " to $0.01 \mathrm{~mm}$ resolution.

3. Record dimension "OAL" to $0.01 \mathrm{~mm}$ resolution. This can be measured with a caliper.

4. Measure $W_{1}, W_{2}$, and $W_{c}$ two times. Reset width reference zero for each measurement (make two independent measurements at each location).

5. Measure thicknesses $T_{1}$ and $T_{2}$, twice at each location, approximately $3 \mathrm{~mm}$ either side of centerline. Measuring device contact surface should not cover the gage mark indent. Ball tip micrometer use is suggested for small contact area on indent face of specimen. Use minimum pressure needed to achieve consistent measurements.

6. Record room temperature before starting and after completion of each group of measurements.

\begin{tabular}{|c|c|c|c|c|c|c|c|c|c|c|c|c|c|c|c|}
\hline \multirow{2}{*}{$\begin{array}{c}\text { Specimen } \\
\text { ID }\end{array}$} & \multirow{2}{*}{$\begin{array}{l}\mathrm{OAL} \\
(\mathrm{mm}) \\
\end{array}$} & \multirow{2}{*}{$\begin{array}{c}\mathrm{C} \\
(\mathrm{mm}) \\
\end{array}$} & \multicolumn{2}{|c|}{$\mathrm{W}_{1}(\mathrm{~mm})$} & \multicolumn{2}{|c|}{$\mathrm{W}_{2}(\mathrm{~mm})$} & \multicolumn{2}{|c|}{$\mathrm{W}_{\mathrm{c}}(\mathrm{mm})$} & \multicolumn{3}{|c|}{$\mathrm{GL}(\mathrm{mm})$} & \multicolumn{2}{|c|}{$T_{1}(\mathrm{~mm})$} & \multicolumn{2}{|c|}{$\mathrm{T}_{2}(\mathrm{~mm})$} \\
\hline & & & $\# 1$ & \#2 & $\# 1$ & \#2 & $\# 1$ & \#2 & $\# 1$ & \#2 & $\# 3$ & $\# 1$ & \#2 & $\# 1$ & $\# 2$ \\
\hline$T I$ & 52.340 & 16.827 & 3.136 & 3.136 & 135 & 3.136 & .122 & 3.121 & 12.732 & 12.722 & 12.721 & .387 & 387 & 386 & 386 \\
\hline$T 2$ & 2.383 & $|4.87|$ & 3.140 & 3.140 & 3.141 & 3.141 & 3.124 & 3.124 & 12.675 & 12.667 & 12.685 & .388 & 388 & .391 & .391 \\
\hline T3 & 52.410 & 16.916 & 3.143 & 3.144 & 3.140 & 3.140 & - & 11 & 12.687 & 12.483 & 12.685 & .389 & .389 & .389 & .389 \\
\hline$T 4$ & 52.442 & 16.925 & 3.151 & 3.152 & 3.144 & 3.144 & 3.117 & 3.117 & 12.676 & 12.675 & 12.480 & 389 & .389 & .391 & .391 \\
\hline TS & 52.474 & 17.010 & 3.217 & 3.218 & 3.184 & 3.184 & 3.179 & 3.179 & 12.756 & 12.740 & $12.72 / 1$ & .389 & .389 & .389 & .389 \\
\hline TG & 52.498 & 17.018 & 3.135 & 3.134 & 3.149 & 3.149 & 3.115 & 3.115 & 12.754 & 12.756 & 12.757 & 389 & .389 & .390 & .390 \\
\hline T1 & 52.523 & 17.095 & 3.137 & 3.137 & 3.138 & 3.138 & 3.112 & 3.112 & 12.728 & 12.723 & 12.730 & .390 & .390 & .390 & .390 \\
\hline 18 & 52.544 & 17.079 & 3.133 & 3.133 & 3.139 & 3.139 & 3.113 & 3.114 & 12.682 & 12.488 & 12.687 & .390 & .390 & .390 & .390 \\
\hline
\end{tabular}


TEM-10300-1

03/01/2012

TECHNICAL EVALUATION

Page B5 of B35

Rev. 03

Title:

Elevated Temperature Tensile Tests on DU-10Mo Rolled Foils

TEV No.: 2194

Rev. No.:

2

Project No.:

25228

Date:

$05 / 02 / 18$

Date: $11-7-13$

Tensile Specimen Dimensional Record Sheet (continuation)

Sheet 2 of 3

Measurement Operator Name: James Reseigh

signature: famer Reungt

\begin{tabular}{|c|c|c|c|c|c|c|c|c|c|c|c|c|c|c|c|}
\hline \multirow{2}{*}{$\begin{array}{c}\text { Specimen } \\
\text { ID }\end{array}$} & \multirow{2}{*}{$\begin{array}{l}\mathrm{OAL} \\
(\mathrm{mm})\end{array}$} & \multirow{2}{*}{$\begin{array}{l}\mathrm{C} \\
(\mathrm{mm})\end{array}$} & \multicolumn{2}{|c|}{$W_{1}(\mathrm{~mm})$} & \multicolumn{2}{|c|}{$W_{2}(\mathrm{~mm})$} & \multicolumn{2}{|c|}{$W_{c}(\mathrm{~mm})$} & \multicolumn{3}{|c|}{$\mathrm{GL}(\mathrm{mm})$} & \multicolumn{2}{|c|}{$T_{1}(\mathrm{~mm})$} & \multicolumn{2}{|c|}{$\mathrm{T}_{2}(\mathrm{~mm})$} \\
\hline & & & $\# 1$ & $\# 2$ & $\# 1$ & $\# 2$ & $\# 1$ & $\# 2$ & $\# 1$ & \#2 & $\# 3$ & $\# 1$ & $\# 2$ & $\# 1$ & \#2 \\
\hline$T q$ & 52.568 & 17.106 & 3.131 & 3.131 & 3.135 & 3.135 & 3.109 & 3.109 & 12.788 & 12.791 & 12.786 & .389 & .389 & .389 & .389 \\
\hline$T 10$ & 52.599 & 17.121 & 3.132 & 3.132 & 3.136 & 3.136 & 3.110 & 3.109 & 12.795 & 12.801 & 12.794 & .391 & 391 & 391 & 391 \\
\hline$T I I$ & 52.637 & 17.180 & 3.133 & 3.134 & 3.132 & 3.132 & 3.115 & 3.115 & 12.695 & 12.706 & 12.700 & .389 & .389 & 391 & 391 \\
\hline$T 12$ & 52.264 & 17.176 & 3.139 & 3.139 & 3.139 & 3.140 & 3.112 & 3.111 & 12.786 & 12.780 & 12.789 & .391 & .391 & .390 & .389 \\
\hline$T 13$ & 52.663 & 17.200 & 3.132 & 3.132 & 3.133 & 3.133 & 3.112 & 3.113 & 12.727 & 12.730 & 12.725 & .386 & .386 & .389 & .389 \\
\hline$T 14$ & 52.677 & 17.211 & 3.1412 & 3.141 & 3.138 & 3.138 & 3.107 & 3.106 & $/ 2.723$ & 12.714 & 12.724 & .386 & .386 & 386 & .386 \\
\hline T15 & 52.478 & 17.123 & 3.069 & 3.068 & 3.090 & 3.090 & 3.049 & 3.050 & 12.717 & 12.710 & 12.722 & 386 & .387 & .388 & .388 \\
\hline T16 & 52.689 & 17.077 & $3.14 / 3$ & 3.143 & 3.144 & 3.147 & 3.133 & 3.134 & 12.688 & 12.684 & 12.690 & 386 & .386 & 386 & 386 \\
\hline$T 17$ & 52.699 & 17.091 & 3.134 & 3.133 & 3.130 & 3.131 & 3.117 & 3.116 & 12.813 & 12.814 & 12.802 & .386 & .386 & .388 & .387 \\
\hline$T 18$ & 52.706 & 17.111 & 3.110 & 3.110 & 3.104 & 3.105 & 3.092 & 3.091 & 12.746 & 12.743 & 12.7410 & .385 & 385 & 386 & 386 \\
\hline$T 19$ & 52.706 & 7.109 & 3.101 & 3.101 & 3.107 & 3.107 & 3.053 & 3.052 & 12.736 & 12.740 & 12.738 & .387 & .387 & .389 & .389 \\
\hline$T 20$ & 51.360 & 15.315 & 3.136 & 3.136 & 3.131 & 3.131 & 3.119 & 3.119 & 12.682 & 12.681 & 12.681 & .389 & .389 & .389 & .389 \\
\hline T21 & 51.361 & 15.302 & 3.136 & 3.137 & 3.140 & 3.140 & 3.129 & 3.128 & 12.780 & 12.782 & 12.782 & 386 & .386 & .386 & 386 \\
\hline$T 22$ & 51.352 & 15.316 & 3.133 & 3.134 & 3.134 & 3.134 & 3.114 & 3.114 & 12.783 & 12.785 & 12.788 & .386 & .386 & 389 & .389 \\
\hline$T 23$ & 51.348 & 15.326 & 3.145 & $3.14 / 5$ & 3.142 & 3.1412 & 3.118 & 3.118 & 12.763 & 12.747 & 12.744 & .386 & .386 & .388 & 388 \\
\hline$T 24$ & 51.355 & 15.333 & 3.188 & 3.188 & 3.208 & 3.208 & 3.172 & 3.172 & 12.720 & 12,725 & 12.725 & .389 & .389 & .389 & .389 \\
\hline$T 25$ & 51.357 & 15.303 & 3.136 & 3.136 & 3.128 & 3.127 & 3.117 & 3.116 & 12839 & 12.838 & 12.840 & 389 & .389 & .389 & .389 \\
\hline T26 & 51.352 & 15.287 & 3.137 & 3.137 & 3.131 & 3.130 & 3.112 & 3.112 & 12.802 & 12.797 & 12.790 & .386 & 386 & 389 & 389 \\
\hline T27 & 51.354 & 15.266 & 3.138 & 3.138 & 3.1 & & 3.109 & 3.109 & 12.703 & 12.705 & 12.701 & .386 & .386 & .389 & 389 \\
\hline$T 28$ & 51.363 & 15.278 & 3.133 & 3.132 & 3.125 & 3.125 & 3.109 & 3.109 & 12.796 & 12.780 & 12.776 & .387 & .387 & .386 & .386 \\
\hline
\end{tabular}

Measurement Notes (use back of sheet if needed):

T18-2 Conical indents on end opposite the Lable end. GL measurement is on the furthest indents apant 
TEM-10300-1

03/01/2012

TECHNICAL EVALUATION

Page B6 of B35

Rev. 03

Title:

Elevated Temperature Tensile Tests on DU-10Mo Rolled Foils

TEV No.:

2194

Rev. No.:

2

Project No.:

25228

Date:

$05 / 02 / 18$

Date: $11-7-13$

Tensile Specimen Dimensional Record Sheet (continuation)

Sheet 3 of 3

Measurement Operator Name: Jamus Reseigh

signature: farmen Rurgish

\begin{tabular}{|c|c|c|c|c|c|c|c|c|c|c|c|c|c|c|c|}
\hline \multirow{2}{*}{$\begin{array}{c}\text { Specimen } \\
\text { ID }\end{array}$} & \multirow{2}{*}{$\begin{array}{l}\text { OAL } \\
(\mathrm{mm}) \\
\end{array}$} & \multirow{2}{*}{$\begin{array}{c}\mathrm{C} \\
(\mathrm{mm}) \\
\end{array}$} & \multicolumn{2}{|c|}{$W_{1}(\mathrm{~mm})$} & \multicolumn{2}{|c|}{$W_{2}(\mathrm{~mm})$} & \multicolumn{2}{|c|}{$W_{c}(m m)$} & \multicolumn{3}{|c|}{$\mathrm{GL}(\mathrm{mm})$} & \multicolumn{2}{|c|}{$T_{1}(\mathrm{~mm})$} & \multicolumn{2}{|c|}{$T_{2}(\mathrm{~mm})$} \\
\hline & & & $\# 1$ & \#2 & $\# 1$ & \#2 & $\# 1$ & $\# 2$ & $\# 1$ & \#2 & $\# 3$ & $\# 1$ & $\# 2$ & $\# 1$ & \#2 \\
\hline T29 & 51.358 & 15.280 & 3.135 & 3.134 & 3.135 & 3.136 & 3.105 & 3.105 & 12.733 & 12.739 & 12.740 & .386 & .386 & .386 & .386 \\
\hline T30 & 51.631 & 15.303 & 3.135 & 3.135 & 3.137 & 3.137 & 3.123 & 3.123 & $12.74 / 5$ & $12.74 / 5$ & 12.752 & .387 & .387 & .389 & .389 \\
\hline$T 31$ & 51.635 & 15.303 & 3.134 & 3.133 & 3.130 & 3.131 & 3.110 & 3.111 & 12.751 & 12.756 & 12.754 & .386 & .386 & .389 & .389 \\
\hline T32 & 51.635 & 15.301 & 3.135 & 3.135 & 3.131 & 3.131 & 3.114 & 3.114 & 12.7410 & 12.7411 & 12.738 & .387 & .387 & 388 & .388 \\
\hline T33 & 51.647 & 15.290 & 3.136 & 3.135 & 3.122 & 3.122 & 3.104 & 3.104 & 12.807 & 12.813 & 12.806 & 386 & .386 & 387 & .387 \\
\hline T34 & 51.650 & 5.341 & 3.090 & 3.089 & 3.073 & 3.073 & 3.0417 & 3.047 & 12.810 & 12.796 & 12.795 & 386 & .386 & .387 & .387 \\
\hline T35 & 51.668 & 15.460 & $3.14 / 5$ & 3.144 & 3.148 & 3.1418 & 3.123 & 3.123 & 12.689 & 12.697 & 12.692 & .389 & .389 & .390 & .390 \\
\hline T36 & 51.682 & 15.479 & 3.127 & 3.127 & 3.132 & 3.131 & 3.104 & 3.105 & 12.647 & 12.648 & 12.681 & 388 & .388 & .391 & .39 \\
\hline T37 & 51.698 & 15.484 & 3.120 & 3.120 & 3.115 & 3.114 & 3.097 & 3.097 & 12.760 & 12.7410 & 12.738 & .387 & .387 & .388 & 388 \\
\hline T38 & 51.700 & 15.542 & 3.106 & 3.105 & 3.112 & 3.113 & 3.051 & 3.052 & 12.729 & 12.723 & 12.721 & 386 & .386 & 388 & .388 \\
\hline & & & & & & & & & & & & & & & \\
\hline & & & & & & & & & & & & & & & \\
\hline & & & & & & & & & & & & & & & \\
\hline & & & & & & & & & & & & & & & \\
\hline & & & & & & & & & & & & & & & \\
\hline & & & & & & & & & & & & & & & \\
\hline & & & & & & & & & & & & & & & \\
\hline & & & & & & & & & & & & & & & \\
\hline & & & & & & & & & & & & & & & \\
\hline & & & & & & & & & & & & & & & \\
\hline
\end{tabular}

Measurement Notes (use back of sheet if needed): 
Title:

$\begin{array}{llll}2194 & \text { Rev. No.: } & 2 & \text { Project No.: }\end{array}$

25228

Date:

$05 / 02 / 18$

Foil 551-2-2 Pretest dimensions old

Date: $10-15-13 \quad$ Tensile Specimen Dimensional Record Sheet

Measurement Operator Name: James Reseigh Signature: Jamus Reseigh

Sheet 1 of 3
GL measurement instrument
ID: $A C>B$
Expiration Date: $4-11-14$
$W$ measurement instrument
ID: $A G>8$
Expiration Date: $4-11-14$
$T$ measurement instrument
ID: Alo9
Expiration Date: $11-12-13$
Temperature instrument
ID: 730725
Expiration Date: $10-1-14$

1. Locations to measure are indicated in the diagram. All dimensions in $\mathrm{mm}$. Record measurements to $0.001 \mathrm{~mm}$.

2. Conical indent marks for GL are small, less than $0.1 \mathrm{~mm}$ typical. Measure indents center-to-center three times with optical comparator. Reset reference zero for each measurement (three independent measurements).

3. Measure $W_{1}, W_{2}$, and $W_{c}$ two times. Reset reference zero for each measurement (two independent measurements).

4. Measure two thicknesses at each of two locations inside of gage mark indents as indicated. Measuring device contact surface should not cover the indent. Ball tip

micrometer use is suggested for small contact area on indent face of specimen. Use minimum pressure needed to achieve consistent measurements.

5. Record room temperature before starting and after completion of each group of measurements.

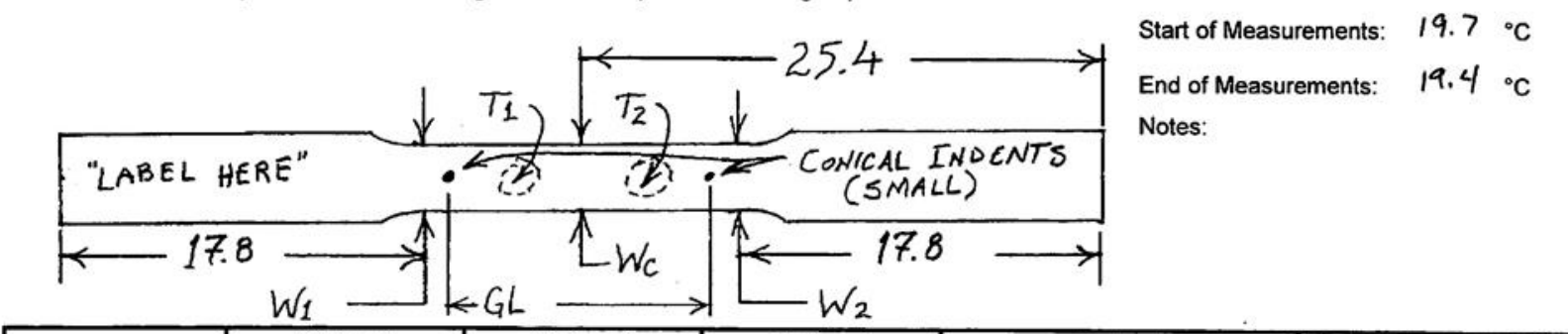

\begin{tabular}{|c|c|c|c|c|c|c|c|c|c|c|c|c|c|}
\hline \multirow[b]{2}{*}{ Specimen ID } & \multicolumn{2}{|c|}{$\mathrm{W}_{1}(\mathrm{~mm})$} & \multicolumn{2}{|c|}{$\mathrm{W}_{2}(\mathrm{~mm})$} & \multicolumn{2}{|c|}{$\mathrm{W}_{c}(\mathrm{~mm})$} & \multicolumn{3}{|c|}{$\mathrm{GL}(\mathrm{mm})$} & \multicolumn{2}{|c|}{$T_{1}(\mathrm{~mm})$} & \multicolumn{2}{|c|}{$T_{2}(\mathrm{~mm})$} \\
\hline & $\# 1$ & $\# 2$ & $\# 1$ & $\# 2$ & $\# 1$ & $\# 2$ & $\# 1$ & $\# 2$ & $\# 3$ & $\# 1$ & $\# 2$ & $\# 1$ & $\# 2$ \\
\hline$\angle 1$ & 3.145 & 3.147 & 3.1414 & 3.1415 & 3.119 & 3.120 & 12.768 & 12.771 & 12.769 & .369 & .369 & .349 & .370 \\
\hline 42 & 3.1418 & 3.148 & 3.152 & 3.152 & 3.130 & 3.130 & 12.703 & 12.712 & 12.703 & .374 & .374 & .377 & .377 \\
\hline L3 & 3.145 & 3.146 & 3.150 & 3.150 & 3.125 & 3.125 & 12.741 & 12.768 & 12.767 & .377 & .377 & .380 & .380 \\
\hline$L 4$ & 3.155 & 3.156 & 3.151 & 3.152 & 3.130 & 3.129 & 12.493 & 12.693 & 12.687 & 378 & .378 & .382 & .381 \\
\hline$\angle 5$ Invaliel & 3.373 & 3.373 & 3.505 & 3.504 & 3.418 & 3.418 & 12.782 & 12.786 & 12.783 & .384 & .383 & 384 & 384 \\
\hline 46 & 3.148 & 3.148 & $3.16 / 5$ & 3.145 & 3.133 & 3.132 & 12.750 & 12.763 & 12.764 & .386 & .387 & .388 & .387 \\
\hline$\angle 7$ & 3.148 & 3.147 & 3.146 & 3.1416 & 3.126 & 3.125 & 12.738 & 12.735 & 12.734 & .388 & .387 & .388 & .390 \\
\hline 48 & 3.1414 & 3.142 & 3.145 & 3.147 & 3.131 & 3.131 & 12.744 & 12.7417 & 12.737 & .389 & .389 & .389 & .389 \\
\hline$\angle 9$ & 3.144 & 3.145 & 3.138 & 3.138 & 3.132 & 3.132 & 12.752 & 12.746 & 12.745 & .389 & .389 & .389 & .388 \\
\hline$\angle 10$ & 3.150 & 3.150 & 3.164 & 3.164 & 3.141 & 3.1410 & $12, x_{1}$ & 12.761 & 12.77 & .386 & .386 & .388 & 388 \\
\hline
\end{tabular}


TEM-10300-1

03/01/2012

TECHNICAL EVALUATION

Page B8 of B35

Rev. 03

Title:

Elevated Temperature Tensile Tests on DU-10Mo Rolled Foils

TEV No.: 2194

Rev. No.:

2

Project No.:

25228

Date:

05/02/18

Date: $10-15-13$

Measurement Operator
Tensile Specimen Dimensional Record Sheet (continuation)

Name: James Reseigh Signature: Jame thumil
Sheet 2 of 3

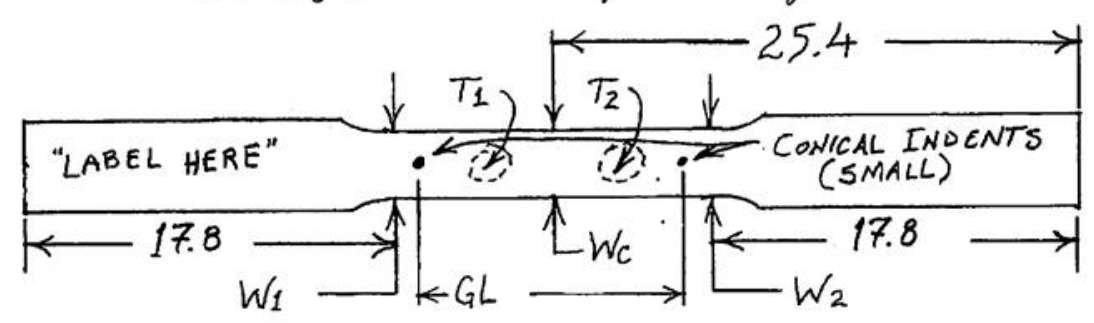

\begin{tabular}{|c|c|c|c|c|c|c|c|c|c|c|c|c|c|}
\hline \multirow[b]{2}{*}{ Specimen ID } & \multicolumn{2}{|c|}{$W_{1}(\mathrm{~mm})$} & \multicolumn{2}{|c|}{$\mathrm{W}_{2}(\mathrm{~mm})$} & \multicolumn{2}{|c|}{$W_{c}(m m)$} & \multicolumn{3}{|c|}{$\mathrm{GL}(\mathrm{mm})$} & \multicolumn{2}{|c|}{$T_{1}(\mathrm{~mm})$} & \multicolumn{2}{|c|}{$T_{2}(\mathrm{~mm})$} \\
\hline & $\# 1$ & $\# 2$ & $\# 1$ & $\# 2$ & $\# 1$ & $\# 2$ & $\# 1$ & $\# 2$ & $\# 3$ & $\# 1$ & $\# 2$ & $\# 1$ & $\# 2$ \\
\hline$\angle 11$ & 3.148 & $3.14 / 9$ & 3.144 & 3.143 & 3.129 & 3.129 & 12.758 & 12.752 & 12.755 & 382 & 382 & 384 & .384 \\
\hline$\angle 12$ & 3.158 & 3.158 & 3.150 & 3.150 & 3.138 & 3.138 & 12.730 & 12.725 & 12.720 & .377 & .377 & 378 & .379 \\
\hline$\angle 13$ & 3.154 & 3.156 & 3.149 & 3.149 & 3.136 & 3.135 & 12.740 & 12.759 & 12.752 & .374 & .374 & .372 & .372 \\
\hline$\angle 29$ & 3.143 & 3.143 & 3.141 & 3.141 & 3.114 & 3.114 & 12.771 & 12.724 & 12.770 & .376 & .377 & .375 & .375 \\
\hline$\angle 30$ & 3.147 & 3.1416 & 3.153 & 3.154 & 3.127 & 3.127 & 12.640 & 12.686 & 12.689 & .381 & 381 & .380 & .380 \\
\hline$\angle 31$ & 3.140 & 3.140 & 3.140 & 3.141 & 3.132 & 3.131 & 12.725 & 12.731 & 12.728 & .385 & .385 & .385 & .384 \\
\hline$\angle 32$ & 3.148 & 3.149 & 3.1419 & 3.148 & 3.129 & 3.129 & $12.7 \times 10$ & 12.747 & 12.740 & .388 & .388 & 388 & .390 \\
\hline$L 33$ Invalid $_{\text {L }}$ & 3.371 & 3.371 & 3.513 & 3.512 & 3.427 & 3.427 & 12.6 .72 & 12.676 & 12.670 & 392 & .392 & .389 & .389 \\
\hline$L 34$ & 3.151 & 3.152 & 3.1419 & 3.149 & 3.129 & 3.128 & 12.753 & $12.74 / 2$ & 12.754 & .391 & .391 & 390 & .390 \\
\hline$\angle 35$ & 3.146 & 3.1416 & 3.151 & 3.150 & 3.131 & 3.130 & 12.629 & 12.637 & 12.634 & .392 & .392 & .311 & .391 \\
\hline$\angle 36$ & 3.148 & 3.149 & 3.147 & 3.1416 & 3.130 & 3.131 & 12.689 & 12.690 & 12.485 & .395 & .395 & .394 & .394 \\
\hline$\angle 37$ & 3.143 & 3.143 & 3.144 & 3.144 & 3.122 & 3.122 & 12.706 & 12.700 & 12.701 & .394 & .394 & .392 & .392 \\
\hline$L 38$ & 3.116 & 3.1417 & 3.158 & 3.158 & $3.14 / 3$ & 3.143 & 12.729 & 12.726 & 12.721 & .3941 & .394 & .393 & 392 \\
\hline$\angle 39$ & 3.143 & 3.1412 & 3.142 & 3.1412 & 3.131 & 3.131 & 12.736 & 12.730 & 12.738 & .388 & 388 & .387 & .388 \\
\hline
\end{tabular}

Notes: 
Rev. 03

Elevated Temperature Tensile Tests on DU-10Mo Rolled Foils

Title:

$$
2194
$$

Rev. No.:

2

Project No.:

25228

Date:

05/02/18

Date: $10-15-13$

.

Measurement Operator
Tensile Specimen Dimensional Record Sheet (continuation)

Name: Jarnes Reseigh signature: Jarm themif

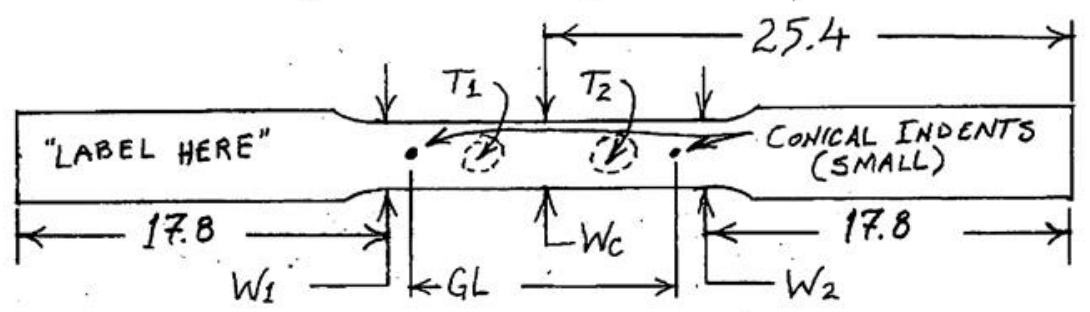

\begin{tabular}{|c|c|c|c|c|c|c|c|c|c|c|c|c|c|}
\hline \multirow{2}{*}{ Specimen ID } & \multicolumn{2}{|c|}{$W_{1}(\mathrm{~mm})$} & \multicolumn{2}{|c|}{$W_{2}(\mathrm{~mm})$} & \multicolumn{2}{|c|}{$W_{c}(\mathrm{~mm})$} & \multicolumn{3}{|c|}{ GL $(\mathrm{mm})$} & \multicolumn{2}{|c|}{$T_{1}(\mathrm{~mm})$} & \multicolumn{2}{|c|}{$T_{2}(\mathrm{~mm})$} \\
\hline & $\#$ & $\# 2$ & $\# 1$ & $\# 2$ & $\# 1$ & $\# 2$ & $\# 1$ & $\# 2$ & $\# 3$ & $\# 1$ & $\# 2$ & $\# 1$ & $\# 2$ \\
\hline$\angle 40$ & 3.154 & 3.154 & 3.158 & 3.157 & 3.146 & 3.147 & 12.756 & 12.75 & 12.741 & .387 & .387 & .384 & .384 \\
\hline$\because 441$ & 3.155 & 3.154 & 3.148 & 3.148 & 3.138 & 3.138 & 12.761 & 12.747 & 12.740 & .378 & .378 & .378 & .378 \\
\hline & & & & & & & & & & & & & \\
\hline$\therefore$ & & & & & & & & & & & & & \\
\hline & & & & & & & & & & & & & \\
\hline & & & & & & & & & & & & & \\
\hline & & & & & & & & & & & & & \\
\hline & & & & & & & & & & & & : & \\
\hline & & & & & & & & & & & & & \\
\hline & & & & & & & & & & & & & \\
\hline & & & & & & & & & & & & & \\
\hline & & & & & & & & & & & & & \\
\hline & & & & & & & & & & & & & \\
\hline$\therefore$ & & & & & & & & & & & & & \\
\hline
\end{tabular}


Rev. 03

\section{Elevated Temperature Tensile Tests on DU-10Mo Rolled Foils}

\section{TEV No.:}

2194

$$
\text { Rev. No.: }
$$

Project No.:

25228

Date:

$05 / 02 / 18$

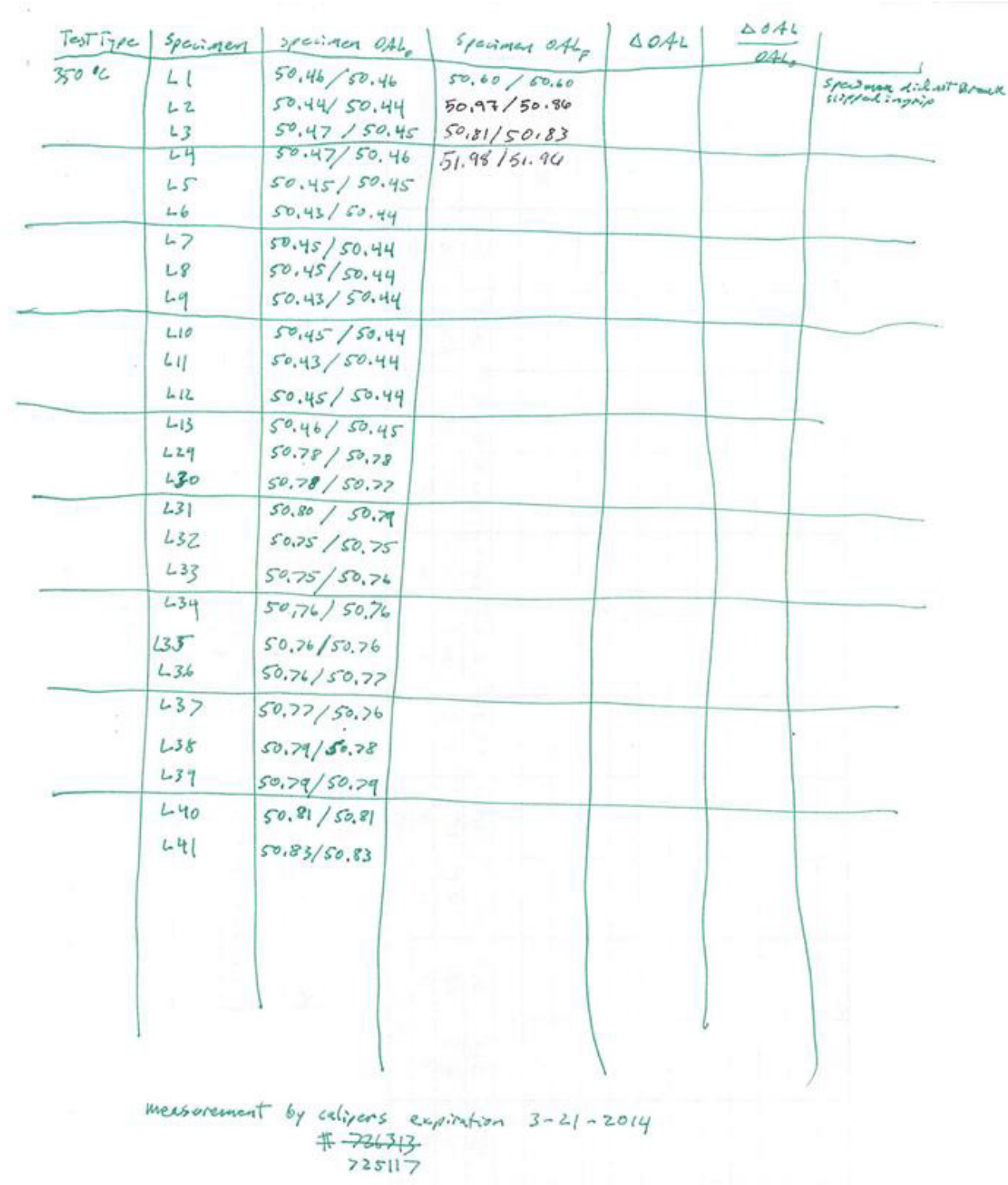


Rev. 03

Title:

TEV No.: 2194

Rev. No.:

2

Project No.:

25228

Date:

$05 / 02 / 18$

Fo: $551-2-2$ Post Test Dimausions

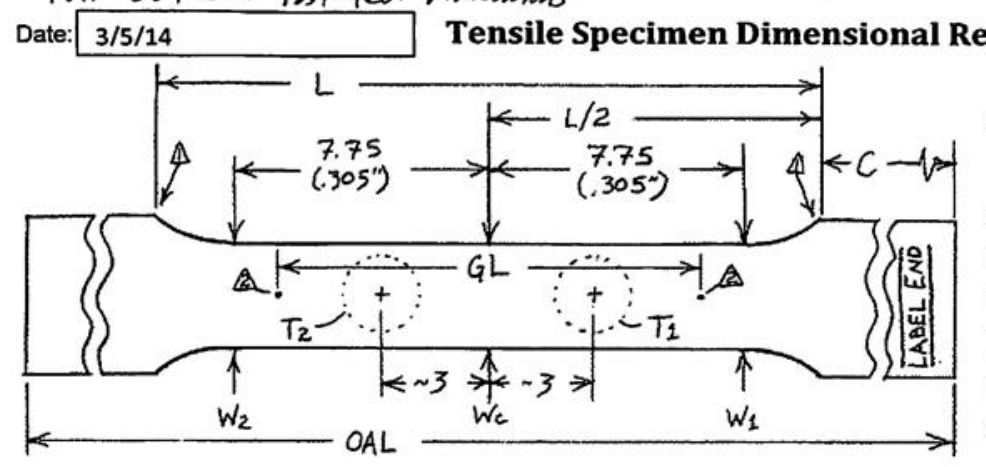

ord Sheet (continuation)

$$
\text { Sheet } 1 \text { of } 3
$$

Measurement Operator Name: James Reseigh

Signature: fosem frovgl

OAL measurement instrument ID: A678

GL measurement instrument ID: A678

Expiration Date: $4 / 11 / 14$

Expiration Date: 4/11/14

W measurement instrument ID: A678

T measurement instrument ID: A609

Temperature instrument ID: A51610

Expiration Date: 4/11/14

Expiration Date: 11/13/14

Start of Measurements: $18.7^{\circ} \mathrm{C}$

Expiration Date: $10 / 22 / 14$

End of Measurements: $19.1^{\circ} \mathrm{C}$

Delta 1. Reference positions for reduced section locations - corner at intersection of end tab and fillet. Locate center between corners (U2) as zero position reference.

Delta 2. Conical indent marks for $\mathrm{GL}$ measurement are small, less than $0.1 \mathrm{~mm}$ typical. Measure indents center-to-center three times with optical comparator. Reset

reference zero for each measurement (three independent measurements).

1. Locations to measure are indicated in the diagram. All dimensions in $\mathrm{mm}$ except as indicated by (in.). Record measurements to $0.001 \mathrm{~mm}$ excepting " $\mathrm{C}$ " and

2. Record dimension "C" to $0.01 \mathrm{~mm}$ resolution.

3. Record dimension "OAL" to $0.01 \mathrm{~mm}$ resolution. This can be measured with a caliper.

4. Measure $W_{1}, W_{2}$, and $W_{c}$ two times. Reset width reference zero for each measurement (make two independent measurements at each location).

Measure thicknesses $\mathrm{T}_{1}$ and $\mathrm{T}_{2}$, twice at each location, approximately $3 \mathrm{~mm}$ either side of centerline. Measuring device contact surface should not cover the gage mark indent. Ball tip micrometer use is suggested for small contact area on indent face of specimen. Use minimum pressure needed to achieve consistent

Reasurements.

temperature before starting and after completion of each group of measurements.

\begin{tabular}{|c|c|c|c|c|c|c|c|c|c|c|c|c|c|c|c|}
\hline \multirow{2}{*}{$\begin{array}{c}\text { Specimen } \\
\text { ID }\end{array}$} & \multirow{2}{*}{$\begin{array}{l}\text { OAL } \\
(\mathrm{mm})\end{array}$} & \multirow{2}{*}{$\begin{array}{c}\mathrm{C} \\
(\mathrm{mm}) \\
\end{array}$} & \multicolumn{2}{|c|}{$\mathrm{W}_{1}(\mathrm{~mm})$} & \multicolumn{2}{|c|}{$\mathrm{W}_{2}(\mathrm{~mm})$} & \multicolumn{2}{|c|}{$\mathrm{W}_{\mathrm{c}}(\mathrm{mm})$} & \multicolumn{3}{|c|}{$\mathrm{GL}(\mathrm{mm})$} & \multicolumn{2}{|c|}{$T_{1}(\mathrm{~mm})$} & \multicolumn{2}{|c|}{$\mathrm{T}_{2}(\mathrm{~mm})$} \\
\hline & & & $\# 1$ & $\# 2$ & $\# 1$ & \#2 & $\# 1$ & $\# 2$ & $\# 1$ & $\# 2$ & $\# 3$ & $\# 1$ & $\# 2$ & $\# 1$ & $\# 2$ \\
\hline $\mathrm{L} 2$ & 51.120 & 15.378 & 3.134 & 3.132 & 3.138 & 3.137 & 3.106 & 3.107 & 13.277 & 13.270 & 13.271 & .378 & .378 & .379 & .379 \\
\hline $\mathrm{L} 3$ & 51.036 & 15.268 & .132 & 3.133 & 3.132 & 3.132 & 3.109 & 3.109 & 13.252 & 13.255 & 13.258 & .381 & .381 & .379 & .379 \\
\hline L4 & 52.129 & 15.406 & .074 & 3.075 & 3.062 & 3.062 & 3.020 & 3.021 & 13.920 & 13.921 & 13.918 & .363 & .363 & .365 & .365 \\
\hline L6 & 51.993 & 15.201 & 3.105 & 3.105 & 3.072 & 3.072 & 3.044 & 3.044 & 13.836 & 13.824 & 13.821 & .365 & .365 & .366 & .366 \\
\hline L7 & 50.709 & 15.152 & 3.153 & 3.154 & 3.143 & 3.144 & 3.129 & 3.130 & 12.823 & 12.821 & 12.822 & .382 & .382 & .382 & .382 \\
\hline *L8 & 62.484 & 15.261 & 3.045 & 3.047 & 3.059 & 3.056 & 2.618 & 2.615 & 22.140 & 22.127 & 22.125 & .258 & .258 & .282 & .282 \\
\hline *L9 & 62.535 & 15.256 & 3.002 & 3.003 & 3.051 & 3.055 & 2.509 & 2.515 & 23.167 & 23.135 & 23.152 & .256 & .256 & .246 & .246 \\
\hline *L10 & 60.445 & 15.192 & 3.015 & 3.014 & 3.014 & 3.014 & 2.744 & 2.748 & 20.946 & 20.928 & 20.929 & .281 & .281 & .272 & .272 \\
\hline
\end{tabular}


Rev. 03

Title:

Elevated Temperature Tensile Tests on DU-10Mo Rolled Foils

TEV No.: 2194

Rev. No.:

2

Project No.:

25228

Date:

$05 / 02 / 18$

Date: $3 / 5 / 14$

Tensile Specimen Dimensional Record Sheet (continuation)

Sheet 2 of 3

Measurement Operator Name: James Reseigh

signature: farnes heugy if

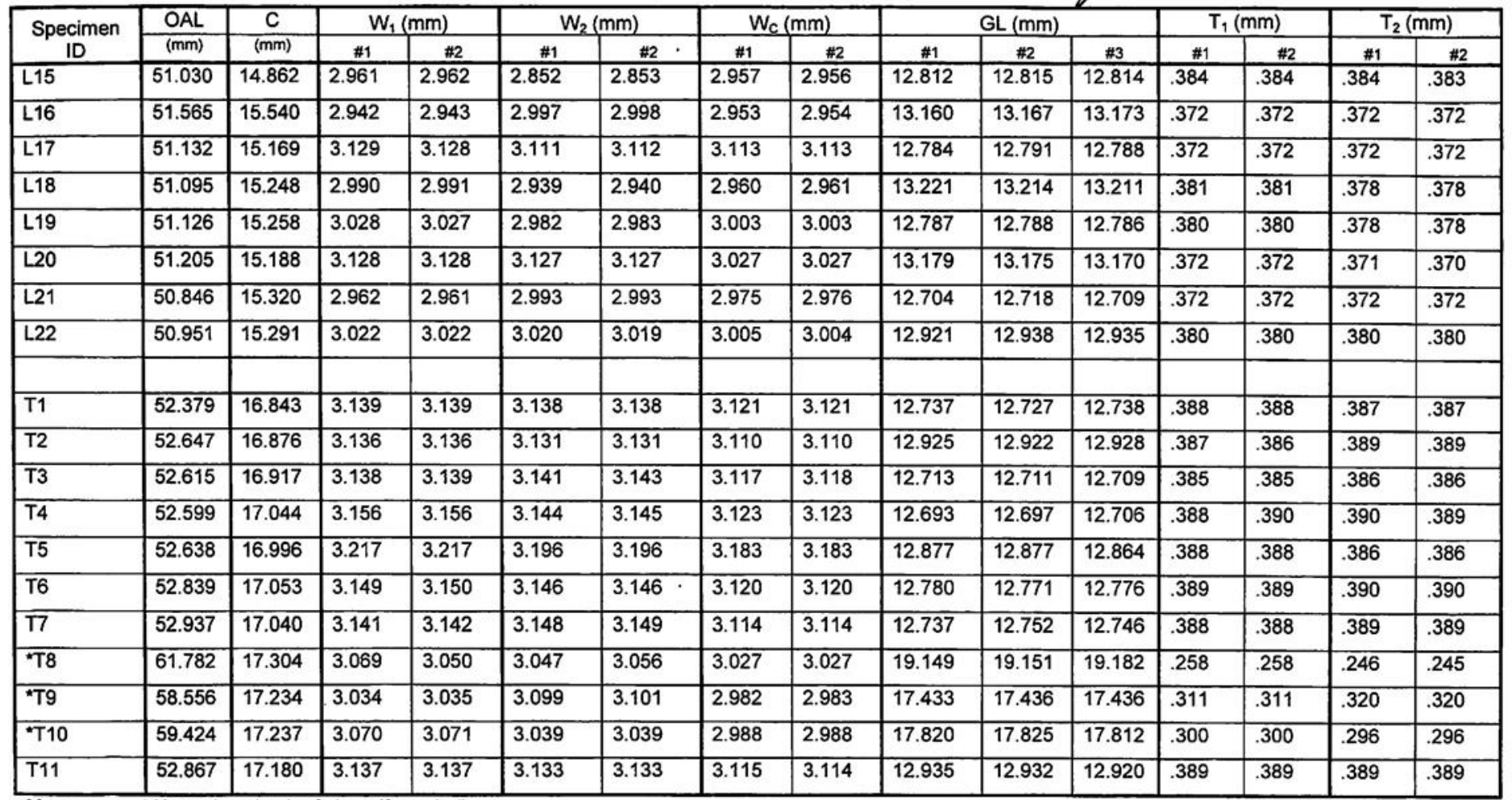

Measurement Notes (use back of sheet if needed):

Note: * Tensile specimens L8, L9, L10, T8, T9, T10, were broke in such a way that it made it very difficult to line the specimens back up for inspection. The Conical indent marks were very faint and difficult to measure. 
Rev. 03

Elevated Temperature Tensile Tests on DU-10Mo Rolled Foils

Title:

$\begin{array}{llll}2194 & \text { Rev. No.: } 2 & \text { Project No.: }\end{array}$

25228

Date:

$05 / 02 / 18$

Date: $3 / 5 / 14$

Tensile Specimen Dimensional Record Sheet (continuation)

Sheet 3 of 3

Measurement Operator Name: James Reseigh

signature: Jame thusyit

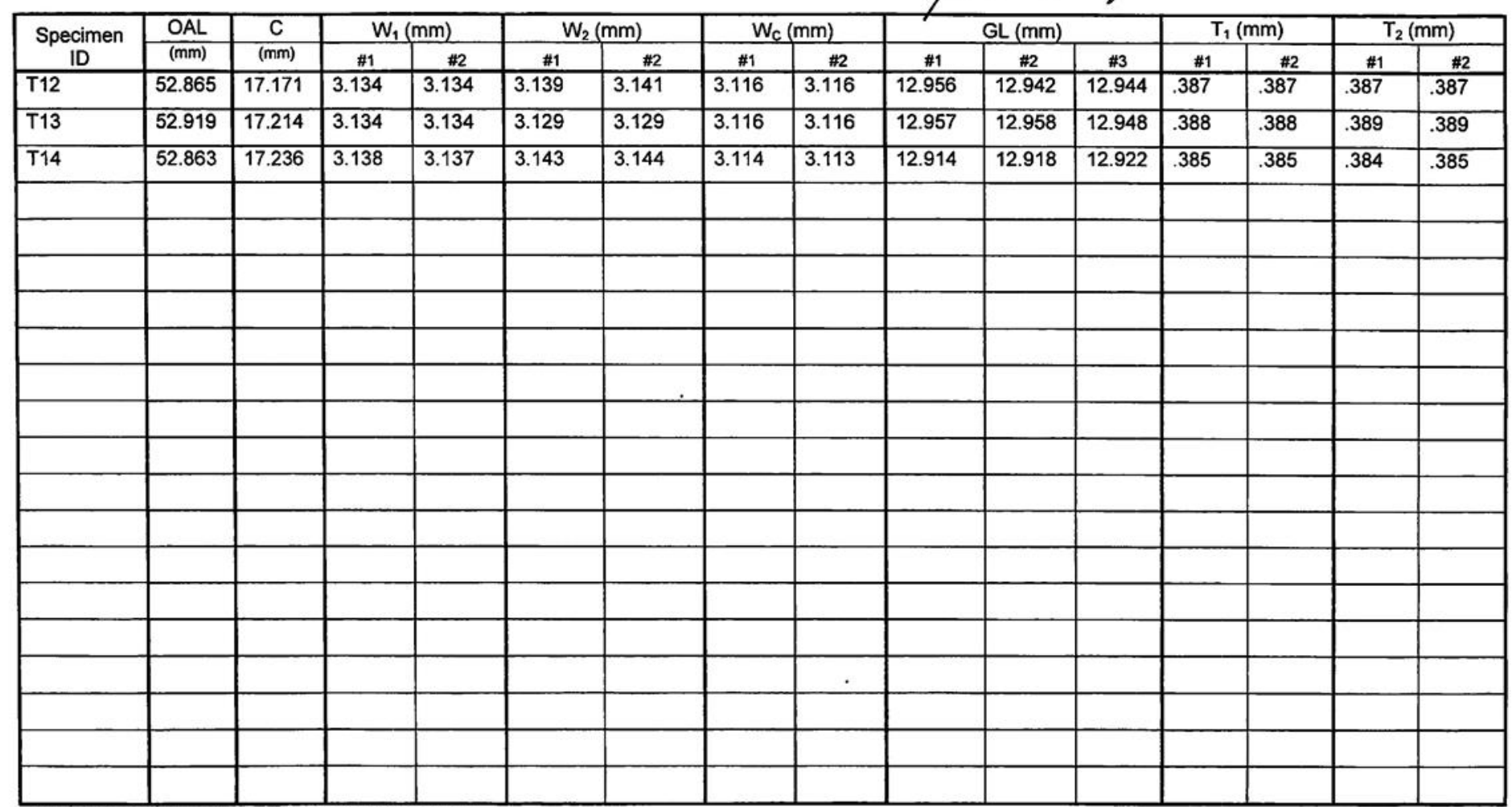

Measurement Notes (use back of sheet if needed): 
Rev. 03

Elevated Temperature Tensile Tests on DU-10Mo Rolled Foils

Title:

2194 Rev. No.: 2 Project No.:

25228

Date:

$05 / 02 / 18$

\begin{tabular}{lccc} 
TEV No.: & 2194 & Rev. No.: & 2 \\
\hline & & Foil $551-2-2$ & rretest vimensions
\end{tabular}

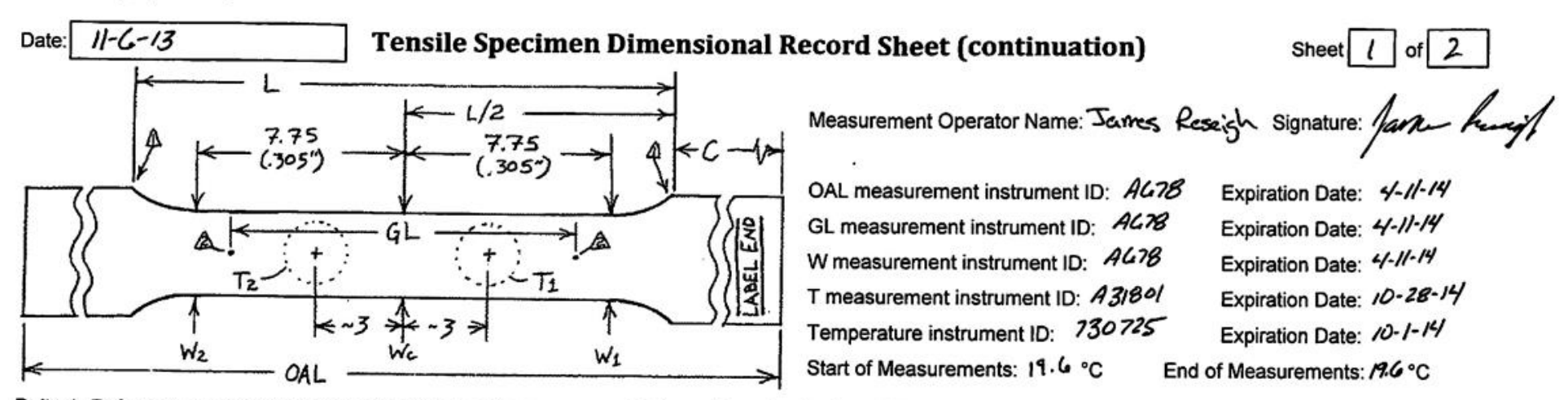

Delta 1. Reference positions for reduced section locations - corner at intersection of end tab and fillet. Locate center between corners (L/2) as zero position reference. It is not necessary to record dimension $L$.

Delta 2. Conical indent marks for GL measurement are small, less than $0.1 \mathrm{~mm}$ typical. Measure indents center-to-center three times with optical comparator. Reset reference zero for each measurement (three independent measurements).

1. Locations to measure are indicated in the diagram. All dimensions in $\mathrm{mm}$ except as indicated by (in.). Record measurements to $0.001 \mathrm{~mm}$ excepting " $\mathrm{C}$ " and
"OAL." "OAL."

2. Record dimension " $\mathrm{C}$ " to $0.01 \mathrm{~mm}$ resolution.

3. Record dimension "OAL" to $0.01 \mathrm{~mm}$ resolution. This can be measured with a caliper.

4. Measure $W_{1}, W_{2}$, and $W_{c}$ two times. Reset width reference zero for each measurement (make two independent measurements at each location).

. Measure thicknesses $\mathrm{T}_{1}$ and $\mathrm{T}_{2}$, twice at each location, approximately $3 \mathrm{~mm}$ either side of centerline. Measuring device contact surface should not cover the gage mark indent. Ball tip micrometer use is suggested for small contact area on indent face of specimen. Use minimum pressure needed to achieve consistent
measurements.

6. Record room temperature before starting and after completion of each group of measurements.

\begin{tabular}{|c|c|c|c|c|c|c|c|c|c|c|c|c|c|c|c|}
\hline \multirow{2}{*}{$\begin{array}{c}\text { Specimen } \\
\text { ID }\end{array}$} & \multirow{2}{*}{$\begin{array}{l}\mathrm{OAL} \\
(\mathrm{mm}) \\
\end{array}$} & \multirow{2}{*}{$\begin{array}{c}\mathrm{C} \\
(\mathrm{mm}) \\
\end{array}$} & \multicolumn{2}{|c|}{$\mathrm{W}_{1}(\mathrm{~mm})$} & \multicolumn{2}{|c|}{$\mathrm{W}_{2}(\mathrm{~mm})$} & \multicolumn{2}{|c|}{$W_{c}(m m)$} & \multicolumn{3}{|c|}{$\mathrm{GL}(\mathrm{mm})$} & \multicolumn{2}{|c|}{$T_{1}(\mathrm{~mm})$} & \multicolumn{2}{|c|}{$T_{2}(\mathrm{~mm})$} \\
\hline & & & $\# 1$ & \#2 & $\# 1$ & \#2 & $\# 1$ & \#2 & $\# 1$ & $\# 2$ & $\# 3$ & $\# 1$ & $\# 2$ & $\# 1$ & $\# 2$ \\
\hline LI & 50.589 & 15.381 & 3.136 & 3.136 & 3.139 & 3.139 & 3.115 & 3.116 & 12.800 & 12.796 & 12.798 & .368 & .368 & .370 & .370 \\
\hline$\llcorner 2$ & & & & & & & & & & & & & & & \\
\hline 13 & & & & & & & & & & & & & & & \\
\hline$L 4$ & & & & & & & & & & & & & & & \\
\hline LS & 50.438 & 15.153 & 3.367 & 3.368 & 3.496 & 3.496 & 3.40 & $3.2 / 07$ & 12.776 & 12.776 & 12.761 & .386 & .386 & .386 & .386 \\
\hline$L 6$ & $50.4 / 38$ & 15.109 & 3.149 & 3.148 & 3.139 & 3.138 & 3.121 & 3.121 & 12.763 & 12.767 & 12.769 & .388 & .388 & .388 & .388 \\
\hline$\llcorner 7$ & 50.436 & 15.074 & 3.135 & 3.135 & 3.142 & 3.142 & 3.124 & 3.124 & 12.741 & 12.737 & 12.731 & .390 & .390 & .391 & .391 \\
\hline 48 & $50.4 / 29$ & 15.061 & $3.14 / 4$ & 3.144 & 3.1418 & 3.148 & 3.137 & 3.137 & 12.734 & 12.737 & 12.735 & 386 & .386 & .387 & .388 \\
\hline
\end{tabular}

4 was tested on 16 oct 2013 so these Dimensions are post test values the rest are pretest nalues 
TEM-10300-1

03/01/2012

Rev. 03

Title:
TECHNICAL EVALUATION

Page B15 of B35

Elevated Temperature Tensile Tests on DU-10Mo Rolled Foils

TEV No.:

Rev. No.:

2

Project No.:

25228

Date:

05/02/18

Date: $\quad / 1-6-13$ Tensile Specimen Dimensional Record Sheet (continuation)

Sheet 2 of 2

Measurement Operator Name: James Reseigh

signature: Jarm hecuagt

\begin{tabular}{|c|c|c|c|c|c|c|c|c|c|c|c|c|c|c|c|}
\hline \multirow{2}{*}{$\begin{array}{c}\text { Specimen } \\
\text { ID }\end{array}$} & \multirow{2}{*}{$\begin{array}{l}\mathrm{OAL} \\
(\mathrm{mm}) \\
\end{array}$} & \multirow{2}{*}{$\begin{array}{c}\mathrm{C} \\
(\mathrm{mm}) \\
\end{array}$} & \multicolumn{2}{|c|}{$W_{1}(\mathrm{~mm})$} & \multicolumn{2}{|c|}{$\mathrm{W}_{2}(\mathrm{~mm})$} & \multicolumn{2}{|c|}{$W_{c}(\mathrm{~mm})$} & \multicolumn{3}{|c|}{$\mathrm{GL}(\mathrm{mm})$} & \multicolumn{2}{|c|}{$T_{1}(\mathrm{~mm})$} & \multicolumn{2}{|c|}{$T_{2}(\mathrm{~mm})$} \\
\hline & & & $\# 1$ & \#2 & $\# 1$ & $\# 2$ & $\# 1$ & $\# 2$ & $\# 1$ & $\# 2$ & $\# 3$ & $\# 1$ & $\# 2$ & $\# 1$ & $\# 2$ \\
\hline & $50.4 / 34$ & 15.009 & 3.160 & 3.160 & 3.137 & 3.137 & 3.129 & 3.129 & 12.741 & 12.7412 & 12.739 & 386 & .386 & .388 & .388 \\
\hline 4.10 & 50.440 & 14.939 & 3.146 & 3.146 & 3.140 & 3.140 & 3.130 & 3.129 & 12.7419 & 12.761 & 12.759 & .386 & .386 & .386 & .386 \\
\hline LII & 50.4137 & 14.944 & 3.136 & 3.136 & 3.1412 & 3.142 & 3.121 & 3.120 & 12.752 & 12.748 & 12.755 & 383 & .383 & .383 & .383 \\
\hline$L 12$ & 50.454 & 14.857 & 3.155 & 3.155 & 3.153 & 3.153 & 3.138 & 3.139 & 12.713 & 12.710 & 12.709 & .378 & .378 & .378 & .378 \\
\hline$L 13$ & 50.466 & 14.844 & 3.154 & 3.154 & 3.146 & 3.145 & 3.136 & 3.136 & 12.743 & 12.743 & 12.741 & 375 & .375 & .371 & .371 \\
\hline$L 29$ & 50.797 & 15.367 & 3.137 & 3.137 & 3.139 & 3.138 & 3.111 & 3.11i & 12.768 & 12.757 & 12.759 & 374 & .374 & .374 & .374 \\
\hline$\angle 30$ & 50.799 & 15.408 & 3.1414 & $3.1 / 14$ & 3.150 & 3.150 & 3.120 & 3.119 & 12.681 & 12.673 & 12.676 & 381 & .381 & .381 & 381 \\
\hline$\angle 31$ & 50.782 & 15.379 & 3.135 & 3.136 & 3.133 & 3.133 & 3.123 & 3.123 & 12.725 & 12.734 & 12.735 & 381 & .381 & .383 & 383 \\
\hline L32 & 50.775 & 15.421 & 3.151 & 3.151 & $3.14 / 5$ & 3.144 & 3.124 & 3.124 & 12.740 & 12.747 & 12.748 & .386 & .386 & .386 & 386 \\
\hline$L 33$ & 50.766 & 15.185 & 3.370 & 3.370 & 3.503 & 3.502 & 3.417 & 3.416 & 12.713 & 12.723 & 12.721 & .391 & .391 & .391 & 391 \\
\hline$\llcorner 34$ & 50.768 & 15.136 & 3.151 & 3.151 & 3.139 & 3.140 & 3.128 & 3.129 & 12.744 & 12.745 & 12.751 & .391 & .391 & .391 & .391 \\
\hline 135 & 50.767 & 15.095 & 3.138 & 3.138 & 3.1415 & 3.145 & 3.128 & 3.127 & 12.609 & 12.612 & 12.606 & .391 & .391 & .392 & .392 \\
\hline$\angle 36$ & 50.770 & 15.081 & 3.147 & 3.147 & 3.1414 & $3.14 / 5$ & 3.134 & 3.135 & 12.687 & 12.674 & 12.679 & .393 & .393 & .391 & .391 \\
\hline$\lcm{37}$ & 50.787 & 15.039 & 3.1414 & 3.144 & 3.141 & 3.140 & 3.124 & 3.123 & 12.716 & 12.710 & 12.718 & .391 & .391 & .391 & .391 \\
\hline$\angle 38$ & 50.789 & 14.984 & 3.152 & 3.152 & 3.150 & 3.150 & 3.139 & 3.139 & 12.713 & 12.718 & 12.720 & .388 & .388 & .388 & 388 \\
\hline 139 & 50.797 & 14.978 & $3.14 / 2$ & $3.1 / 2$ & 3.139 & 3.139 & 3.128 & 3.127 & 12.736 & 12.734 & 12.738 & .387 & 387 & .386 & .386 \\
\hline$L 40$ & 50.815 & 14.926 & 3.161 & 3.160 & 3.156 & 3.156 & 3.138 & 3.138 & 12.731 & 12.742 & 12.743 & .384 & .384 & .381 & 381 \\
\hline$L 4 I$ & 50.839 & 14.903 & 3.164 & 3.164 & 3.1417 & 3.147 & 3.133 & 3.133 & 12.745 & 12.735 & 12.740 & .376 & .376 & .376 & $3 \pi k$ \\
\hline & & & & & & & & & & & & & & & \\
\hline & & & & & & & & & & & & & & & \\
\hline
\end{tabular}


Title:

$$
\begin{array}{llll}
2194 & \text { Rev. No.: } 2 & 2 & \text { Project No.: }
\end{array}
$$

25228

Date:

$05 / 02 / 18$

Foil 551-3 Pre-Test Dimensions

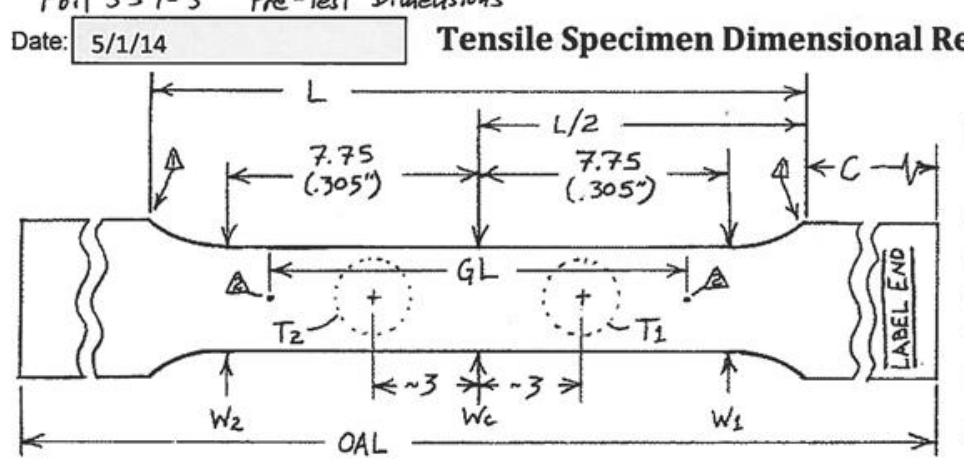

\section{ecord Sheet (continuation)}

Measurement Operator Name: James Reseigh

OAL measurement instrument ID: A678

GL measurement instrument ID: $A 678$

W measurement instrument ID: $A 678$

T measurement instrument ID: $A 609$

Temperature instrument ID: $A 52393$

Start of Measurements: $18.4 \mathrm{C}^{\circ}$
Sheet 1 of 2

Expiration Date: $4 / 9 / 15$

Expiration Date: 4/9/15

Expiration Date: 4/9/15

Expiration Date: 11/13/14

Expiration Date: 6/24/14

End of Measurements: $18.3 \mathrm{C}^{\circ}$

Delta 1. Reference positions for reduced section locations - corner at intersection of end tab and fillet. Locate center between corners (L2) as zero position reference. It is not necessary to record dimension $\mathrm{L}$.

Delta 2. Conical indent marks for $G L$ measurement are small, less than $0.1 \mathrm{~mm}$ typical. Measure indents center-to-center three times with optical comparator. Reset reference zero for each measurement (three independent measurements).

1. Locations to measure are indicated in the diagram. All dimensions in mm except as indicated by (in.). Record measurements to $0.001 \mathrm{~mm}$ excepting " $\mathrm{C}$ " and "OAL."

2. Record dimension "C" to $0.01 \mathrm{~mm}$ resolution.

3. Record dimension "OAL" to $0.01 \mathrm{~mm}$ resolution. This can be measured with a caliper.

4. Measure $W_{1}, W_{2}$, and $W_{c}$ two times. Reset width reference zero for each measurement (make two independent measurements at each location).

\begin{tabular}{|c|c|c|c|c|c|c|c|c|c|c|c|c|c|c|c|}
\hline \multirow{2}{*}{$\begin{array}{c}\text { Specimen } \\
\text { ID }\end{array}$} & \multirow{2}{*}{$\begin{array}{l}\text { OAL } \\
(\mathrm{mm}) \\
\end{array}$} & \multirow{2}{*}{$\begin{array}{c}\mathrm{C} \\
(\mathrm{mm}) \\
\end{array}$} & \multicolumn{2}{|c|}{$\mathrm{W}_{1}(\mathrm{~mm})$} & \multicolumn{2}{|c|}{$\mathrm{W}_{2}(\mathrm{~mm})$} & \multicolumn{2}{|c|}{$W_{c}(\mathrm{~mm})$} & \multicolumn{3}{|c|}{$\mathrm{GL}(\mathrm{mm})$} & \multicolumn{2}{|c|}{$\mathrm{T}_{1}(\mathrm{~mm})$} & \multicolumn{2}{|c|}{$\mathrm{T}_{2}(\mathrm{~mm})$} \\
\hline & & & $\# 1$ & \#2 & $\# 1$ & $\# 2$ & $\# 1$ & \#2 & $\# 1$ & $\# 2$ & $\# 3$ & $\# 1$ & $\# 2$ & $\# 1$ & $\# 2$ \\
\hline L29 & 50.695 & 15.182 & 3.194 & 3.195 & 3.195 & 3.195 & 3.175 & 3.175 & 12.705 & 12.720 & 12.714 & .374 & .376 & .376 & .376 \\
\hline L30 & 50.690 & 15.354 & 3.198 & 3.198 & 3.195 & 3.195 & 3.174 & 3.174 & 12.712 & 12.710 & 12.706 & .383 & .383 & .382 & .382 \\
\hline L31 & 50.688 & 15.377 & 3.190 & 3.190 & 3.189 & 3.189 & 3.168 & 3.168 & 12.752 & 12.751 & 12.748 & .384 & .384 & .383 & .383 \\
\hline L32 & 50.696 & 15.348 & 3.192 & 3.193 & 3.189 & 3.189 & 3.175 & 3.175 & 12.709 & 12.708 & 12.701 & .388 & .388 & .389 & .389 \\
\hline L33 & 50.692 & 15.361 & 3.196 & 3.195 & 3.193 & 3.194 & 3.166 & 3.166 & 12.710 & 12.706 & 12.700 & .390 & .390 & .391 & .391 \\
\hline L34 & 50.688 & 15.329 & 3.190 & 3.190 & 3.190 & 3.191 & 3.167 & 3.167 & 12.770 & 12.774 & 12.780 & .390 & .390 & .390 & .391 \\
\hline L35 & 50.683 & 15.322 & 3.191 & 3.191 & 3.196 & 3.195 & 3.161 & 3.162 & 12.666 & 12.669 & 12.661 & .391 & .392 & .392 & .391 \\
\hline L36 & 50.682 & 15.293 & 3.190 & 3.190 & 3.189 & 3.189 & 3.173 & 3.174 & 12.753 & 12.754 & 12.758 & .393 & .393 & .393 & .393 \\
\hline
\end{tabular}

5. Measure thicknesses $\mathrm{T}_{1}$ and $\mathrm{T}_{2}$, twice at each location, approximately $3 \mathrm{~mm}$ either side of centerline. Measuring device contact surface should not cover the gage mark indent. Ball tip micrometer use is suggested for small contact area on indent face of specimen. Use minimum pressure needed to achieve consistent

6. Record room temperature before starting and after completion of each group of measurements. 
Rev. 03

Elevated Temperature Tensile Tests on DU-10Mo Rolled Foils

$\begin{array}{lllllll}2194 & \text { Rev. No.: } & 2 & \text { Project No.: } & 25228 & \text { Date: } & 05 / 02 / 18\end{array}$

$$
\text { Date: } 5 / 1 / 14
$$

Tensile Specimen Dimensional Record Sheet (continuation)

measurement Operator Name: James Reseigh

Signature:

\begin{tabular}{|c|c|c|c|c|c|c|c|c|c|c|c|c|c|c|c|}
\hline \multirow{2}{*}{$\begin{array}{c}\text { Specimen } \\
\text { ID }\end{array}$} & \multirow{2}{*}{$\begin{array}{l}\mathrm{OAL} \\
(\mathrm{mm})\end{array}$} & \multirow{2}{*}{$\begin{array}{c}\mathrm{C} \\
(\mathrm{mm})\end{array}$} & \multicolumn{2}{|c|}{$W_{1}(\mathrm{~mm})$} & \multicolumn{2}{|c|}{$\mathrm{W}_{2}(\mathrm{~mm})$} & \multicolumn{2}{|c|}{$\mathrm{W}_{\mathrm{c}}(\mathrm{mm})$} & \multicolumn{3}{|c|}{$\mathrm{GL}(\mathrm{mm})$} & \multicolumn{2}{|c|}{$T_{1}(\mathrm{~mm})$} & \multicolumn{2}{|c|}{$\mathrm{T}_{2}(\mathrm{~mm})$} \\
\hline & & & $\# 1$ & $\# 2$ & $\# 1$ & $\# 2$ & $\# 1$ & $\# 2$ & $\# 1$ & $\# 2$ & $\# 3$ & $\# 1$ & $\# 2$ & $\# 1$ & \#2 \\
\hline L37 & 50.692 & 15.256 & 3.190 & 3.190 & 3.192 & 3.192 & 3.171 & 3.170 & 12.769 & 12.772 & 12.771 & .390 & .390 & .391 & .391 \\
\hline L38 & 50.698 & 15.325 & 3.200 & 3.200 & 3.196 & 3.195 & 3.167 & 3.167 & 12.728 & 12.730 & 12.738 & .387 & .387 & .387 & .387 \\
\hline L39 & 50.698 & 15.298 & 3.203 & 3.204 & 3.194 & 3.194 & 3.184 & 3.183 & 12.684 & 12.681 & 12.687 & .387 & .386 & .387 & .387 \\
\hline L40 & 50.700 & 15.280 & 3.184 & 3.184 & 3.185 & 3.186 & 3.169 & 3.169 & 12.720 & 12.721 & 12.725 & .381 & .381 & .381 & .381 \\
\hline L41 & 50.705 & 15.294 & 3.174 & 3.174 & 3.184 & 3.184 & 3.165 & 3.165 & 12.645 & 12.641 & 12.641 & .378 & 378 & .377 & .378 \\
\hline & & & & & & & & & & & & & & & \\
\hline & & & & & & & & & & & & & & & \\
\hline & & & & & & & & & & & & & & & \\
\hline & & & & & & & & & & & & & & & \\
\hline & & & & & & & & & & & & & & & \\
\hline & & & & & & & & & & & & & & & \\
\hline & & & & & & & & & & & & & & & \\
\hline & & & & & & & & & & & & & & & \\
\hline & & & & & & & & & & & & & & & \\
\hline & & & & & & & & & & & & & & & \\
\hline & & & & & & & & & & & & & & & \\
\hline & & & & & & & & & & & & & & & \\
\hline & & & & & & & & & & & & & & & \\
\hline & & & & & & & & & & & & & & & \\
\hline & & & & & & & & & & & & & & & \\
\hline
\end{tabular}

Measurement Notes (use back of sheet if needed):

$$
\text { Sheet } 2 \text { of } 2
$$


Rev. 03

\title{
Elevated Temperature Tensile Tests on DU-10Mo Rolled Foils
}

Title:

\author{
2194 \\ Rev. No.: \\ 2 \\ Project No.:
}

25228

Date:

$05 / 02 / 18$

Foil 551-3 Pre-Test Dimousions

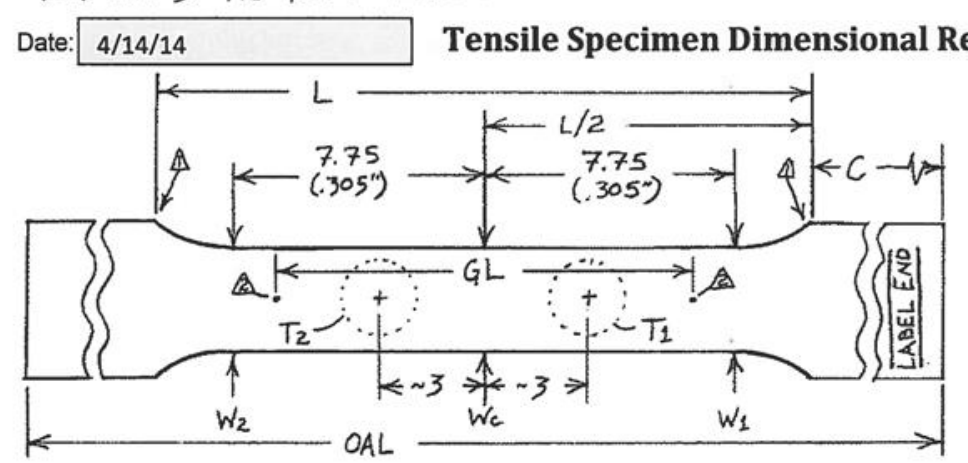

ecord Sheet (continuation)

Measurement Operator Name: James Reseigh

OAL measurement instrument ID: A678

GL measurement instrument ID: A678

W measurement instrument ID: $A 678$

T measurement instrument ID: $A 609$

Temperature instrument ID: $A 51610$

Start of Measurements: $64.3 C^{*}$
Sheet 1 of 3

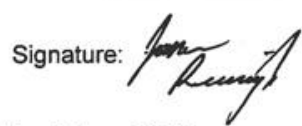

Expiration Date: 4/9/15

Expiration Date: 4/9/15

Expiration Date: 4/9/15

Expiration Date: 11/13/14

Expiration Date: 10/22/14

End of Measurements: $67.8 \mathrm{C}^{\circ}$

Delta 1. Reference positions for reduced section locations - corner at intersection of end tab and fillet. Locate center between corners (U/2) as zero position reference.

It is not necessary to record dimension $\mathrm{L}$.

reference zero for each measurement (three independent measurements).

2. Record dimension " $C$ " to $0.01 \mathrm{~mm}$ resolution.

3. Record dimension "OAL" to $0.01 \mathrm{~mm}$ resolution. This can be measured with a caliper.

4. Measure $W_{1}, W_{2}$, and $W_{c}$ two times. Reset width reference zero for each measurement (make two independent measurements at each location).

5. Measure thicknesses $\mathrm{T}_{1}$ and $\mathrm{T}_{2}$, twice at each location, approximately $3 \mathrm{~mm}$ either side of centerline. Measuring device contact surface should not cover the gage mark indent. Ball tip micrometer use is suggested for small contact area on indent face of specimen. Use minimum pressure needed to achieve consistent measurements.

6. Record room temperature before starting and after completion of each group of measurements.

\begin{tabular}{|c|c|c|c|c|c|c|c|c|c|c|c|c|c|c|c|}
\hline \multirow{2}{*}{$\begin{array}{l}\text { Specimen } \\
\text { ID }\end{array}$} & \multirow{2}{*}{$\begin{array}{l}\text { OAL } \\
(\mathrm{mm})\end{array}$} & \multirow{2}{*}{$\frac{\mathrm{C}}{(\mathrm{mm})}$} & \multicolumn{2}{|c|}{$\mathrm{W}_{1}(\mathrm{~mm})$} & \multicolumn{2}{|c|}{$\mathrm{W}_{2}(\mathrm{~mm})$} & \multicolumn{2}{|c|}{$\mathrm{W}_{\mathrm{c}}(\mathrm{mm})$} & \multicolumn{3}{|c|}{$\mathrm{GL}(\mathrm{mm})$} & \multicolumn{2}{|c|}{$T_{1}(\mathrm{~mm})$} & \multicolumn{2}{|c|}{$\mathrm{T}_{2}(\mathrm{~mm})$} \\
\hline & & & $\# 1$ & $\# 2$ & $\# 1$ & $\# 2$ & $\# 1$ & $\# 2$ & $\# 1$ & $\# 2$ & $\# 3$ & $\# 1$ & $\# 2$ & $\# 1$ & $\# 2$ \\
\hline L1 & 50.490 & 15.154 & 3.200 & 3.199 & 3.196 & 3.196 & 3.166 & 3.165 & 12.732 & 12.739 & 12.742 & .366 & .366 & .365 & .365 \\
\hline L2 & 50.456 & 15.081 & 3.196 & 3.195 & 3.202 & 3.202 & 3.190 & 3.190 & 12.713 & 12.712 & 12.703 & .369 & .369 & .371 & .371 \\
\hline L3 & 50.452 & 15.099 & 3.193 & 3.193 & 3.201 & 3.202 & 3.171 & 3.171 & 12.687 & 12.690 & 12.699 & .377 & .377 & .375 & .375 \\
\hline L4 & 50.441 & 15.086 & 3.194 & 3.194 & 3.186 & 3.187 & 3.167 & 3.166 & 12.753 & 12.765 & 12.750 & .379 & .379 & .381 & .381 \\
\hline L5 & 50.438 & 15.104 & 3.189 & 3.189 & 3.196 & 3.197 & 3.184 & 3.183 & 12.697 & 12.691 & 12.694 & .385 & .385 & .386 & .386 \\
\hline L6 & 50.440 & 15.078 & 3.193 & 3.192 & 3.191 & 3.191 & 3.166 & 3.166 & 12.672 & 12.672 & 12.679 & .387 & .387 & .386 & .386 \\
\hline L7 & 50.436 & 15.083 & 3.188 & 3.188 & 3.197 & 3.196 & 3.171 & 3.169 & 12.737 & 12.738 & 12.732 & .386 & .386 & .385 & .385 \\
\hline L8 & 50.437 & 15.113 & 3.204 & 3.205 & 3.193 & 3.193 & 3.184 & 3.183 & 12.747 & 12.746 & 12.757 & .387 & .387 & .387 & .387 \\
\hline
\end{tabular}


Rev. 03

Title:

Elevated Temperature Tensile Tests on DU-10Mo Rolled Foils

TEV No.: 2194

Rev. No.:

2

Project No.:

25228

Date:

05/02/18

Date: $4 / 14 / 14$

Tensile Specimen Dimensional Record Sheet (continuation)

Sheet 2 of 3

Measurement Operator Name: James Reseigh

Signature:

$\mathrm{GL}(\mathrm{mm}$

\begin{tabular}{|c|c|c|c|c|c|c|c|c|c|c|c|c|c|c|c|}
\hline \multirow{2}{*}{$\begin{array}{l}\text { Specimen } \\
\text { ID }\end{array}$} & \multirow{2}{*}{$\begin{array}{l}\text { OAL } \\
(\mathrm{mm})\end{array}$} & \multirow{2}{*}{$\begin{array}{c}\mathrm{C} \\
(\mathrm{mm})\end{array}$} & \multicolumn{2}{|c|}{$\mathrm{W}_{1}(\mathrm{~mm})$} & \multicolumn{2}{|c|}{$\mathrm{W}_{2}(\mathrm{~mm})$} & \multicolumn{2}{|c|}{$\mathrm{W}_{\mathrm{c}}(\mathrm{mm})$} & \multicolumn{3}{|c|}{$\mathrm{GL}(\mathrm{mm})$} & \multicolumn{2}{|c|}{$\mathrm{T}_{1}(\mathrm{~mm})$} & \multicolumn{2}{|c|}{$\mathrm{T}_{2}(\mathrm{~mm})$} \\
\hline & & & $\# 1$ & \#2 & $\# 1$ & $\# 2$ & $\# 1$ & $\# 2$ & $\# 1$ & $\# 2$ & $\# 3$ & $\# 1$ & \#2 & $\# 1$ & $\# 2$ \\
\hline L9 & 50.440 & 15.104 & 3.191 & 3.192 & 3.187 & 3.188 & 3.164 & 3.165 & 12.688 & 12.682 & 12.680 & .386 & .386 & .386 & .386 \\
\hline L10 & 50.432 & 15.091 & 3.195 & 3.194 & 3.191 & 3.191 & 3.174 & 3.174 & 12.727 & 12.731 & 12.732 & .384 & .384 & .384 & .384 \\
\hline L11 & 50.422 & 15.093 & 3.199 & 3.199 & 3.186 & 3.185 & 3.167 & 3.167 & 12.768 & 12.776 & 12.778 & .382 & .382 & .382 & .382 \\
\hline L12 & 50.415 & 15.080 & 3.187 & 3.186 & 3.196 & 3.196 & 3.183 & 3.184 & 12.690 & 12.690 & 12.688 & .378 & .378 & .378 & .378 \\
\hline L13 & 50.416 & 15.084 & 3.195 & 3.195 & 3.193 & 3.193 & 3.186 & 3.187 & 12.714 & 12.713 & 12.717 & .371 & .371 & .373 & .373 \\
\hline T1 & 50.535 & 15.075 & 3.200 & 3.199 & 3.186 & 3.185 & 3.175 & 3.175 & 12.669 & 12.666 & 12.670 & .387 & .387 & .388 & .388 \\
\hline T2 & 50.542 & 15.093 & 3.199 & 3.200 & 3.195 & 3.195 & 3.177 & 3.177 & 12.686 & 12.691 & 12.682 & .387 & .387 & .386 & .386 \\
\hline T3 & 50.536 & 15.085 & 3.192 & 3.192 & 3.195 & 3.195 & 3.164 & 3.164 & 12.794 & 12.776 & 12.781 & .388 & .388 & .389 & .389 \\
\hline T4 & 50.529 & 15.051 & 3.194 & 3.194 & 3.189 & 3.190 & 3.176 & 3.176 & 12.712 & 12.711 & 12.718 & .388 & .388 & .389 & .389 \\
\hline T5 & 50.526 & 15.050 & 3.186 & 3.187 & 3.189 & 3.189 & 3.169 & 3.169 & 12.741 & 12.742 & 12.749 & .386 & .386 & .388 & .388 \\
\hline T6 & 50.529 & 15.066 & 3.184 & 3.184 & 3.190 & 3.190 & 3.162 & 3.161 & 12.689 & 12.696 & 12.694 & .387 & .387 & .390 & .390 \\
\hline T7 & 50.508 & 15.030 & 3.188 & 3.188 & 3.186 & 3.187 & 3.160 & 3.159 & 12.774 & 12.778 & 12.771 & .387 & .387 & .390 & .390 \\
\hline T8 & 50.493 & 15.004 & 3.191 & 3.191 & 3.193 & 3.192 & 3.167 & 3.168 & 12.643 & 12.640 & 12.638 & .390 & .390 & .390 & .390 \\
\hline T9 & 50.493 & 15.004 & 3.191 & 3.191 & 3.194 & 3.194 & 3.167 & 3.167 & 12.623 & 12.633 & 12.629 & .389 & .389 & .387 & .387 \\
\hline T10 & 50.472 & 14.988 & 3.185 & 3.186 & 3.189 & 3.189 & 3.161 & 3.161 & 12.861 & 12.850 & 12.856 & .389 & .389 & .388 & .388 \\
\hline T11 & 50.462 & 14.990 & 3.192 & 3.192 & 3.188 & 3.188 & 3.167 & 3.167 & 12.729 & 12.729 & 12.739 & .390 & .389 & .390 & .390 \\
\hline T12 & 50.456 & 14.946 & 3.189 & 3.189 & 3.195 & 3.196 & 3.169 & 3.169 & 12.730 & 12.728 & 12.730 & .388 & .389 & .391 & .391 \\
\hline T13 & 50.442 & 14.916 & 3.196 & 3.196 & 3.194 & 3.193 & 3.165 & 3.165 & 12.717 & 12.729 & 12.716 & .390 & .390 & .390 & .390 \\
\hline T14 & 50.435 & 14.908 & 3.180 & 3.180 & 3.190 & 3.190 & 3.167 & 3.166 & 12.749 & 12.756 & 12.747 & .390 & .390 & .391 & .391 \\
\hline
\end{tabular}

Measurement Notes (use back of sheet if needed): 
Rev. 03

Elevated Temperature Tensile Tests on DU-10Mo Rolled Foils

TEV No.:

Rev. No.:

2

Project No.:

25228

Date:

$05 / 02 / 18$

Date: $4 / 14 / 14$

Tensile Specimen Dimensional Record Sheet (continuation)

Sheet 3 of 3

Measurement Operator Name: James Reseigh

Signature:

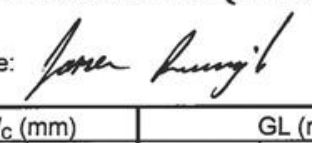

\begin{tabular}{|c|c|c|c|c|c|c|c|c|c|c|c|c|c|c|c|}
\hline \multirow{2}{*}{$\begin{array}{l}\text { Specimen } \\
\text { ID }\end{array}$} & \multirow{2}{*}{$\begin{array}{l}\text { OAL } \\
(\mathrm{mm})\end{array}$} & \multirow{2}{*}{$\frac{\mathrm{C}}{(\mathrm{mm})}$} & \multicolumn{2}{|c|}{$\mathrm{W}_{1}(\mathrm{~mm})$} & \multicolumn{2}{|c|}{$\mathrm{W}_{2}(\mathrm{~mm})$} & \multicolumn{2}{|c|}{$\mathrm{W}_{\mathrm{c}}(\mathrm{mm})$} & \multicolumn{3}{|c|}{$\mathrm{GL}(\mathrm{mm})$} & \multicolumn{2}{|c|}{$T_{1}(\mathrm{~mm})$} & \multicolumn{2}{|c|}{$T_{2}(\mathrm{~mm})$} \\
\hline & & & $\# 1$ & $\# 2$ & $\# 1$ & $\# 2$ & $\# 1$ & \#2 & $\# 1$ & \#2 & $\# 3$ & $\# 1$ & \#2 & $\# 1$ & $\# 2$ \\
\hline T15 & 50.467 & 14.898 & 3.197 & 3.198 & 3.199 & 3.199 & 3.186 & 3.186 & 12.870 & 12.868 & 12.865 & .390 & .389 & .390 & .390 \\
\hline T16 & 50.478 & 14.920 & 3.193 & 3.193 & 3.185 & 3.184 & 3.168 & 3.169 & 12.736 & 12.742 & 12.752 & .389 & .389 & .390 & .391 \\
\hline T17 & 50.476 & 14.878 & 3.183 & 3.181 & 3.192 & 3.193 & 3.156 & 3.156 & 12.721 & 12.727 & 12.727 & .388 & .388 & .389 & .389 \\
\hline T18 & 50.478 & 14.886 & 3.190 & 3.190 & 3.186 & 3.186 & 3.165 & 3.164 & 12.722 & 12.726 & 12.737 & .389 & .389 & .390 & .389 \\
\hline T19 & 50.467 & 14.862 & 3.209 & 3.210 & 3.194 & 3.193 & 3.162 & 3.163 & 12.816 & 12.815 & 12.812 & .389 & .390 & .390 & .388 \\
\hline & & & & & & & & & & & & & & & \\
\hline & & & & & & & & & & & & & & & \\
\hline & & & & & & & & & & & & & & & \\
\hline & & & & & & & & & & & & & & & \\
\hline & & & & & & & & & & & & & & & \\
\hline & & & & & & & & & & & & & & & \\
\hline & & & & & & & & & & & & & & & \\
\hline & & & & & & & & & & & & & & & \\
\hline & & & & & & & & & & & & & & & \\
\hline & & & & & & & & & & & & & & & \\
\hline & & & & & & & & & & & & & & & \\
\hline & & & & & & & & & & & & & & & \\
\hline & & & & & & & & & & & & & & & \\
\hline & & & & & & & & & & & & & & & \\
\hline & & & & & & & & & & & & & & & \\
\hline
\end{tabular}

Measurement Notes (use back of sheet if needed): 
Rev. 03

Title:

TEV No.: 2194

Rev. No.:

2

Project No.:

25228

Date:

$05 / 02 / 18$

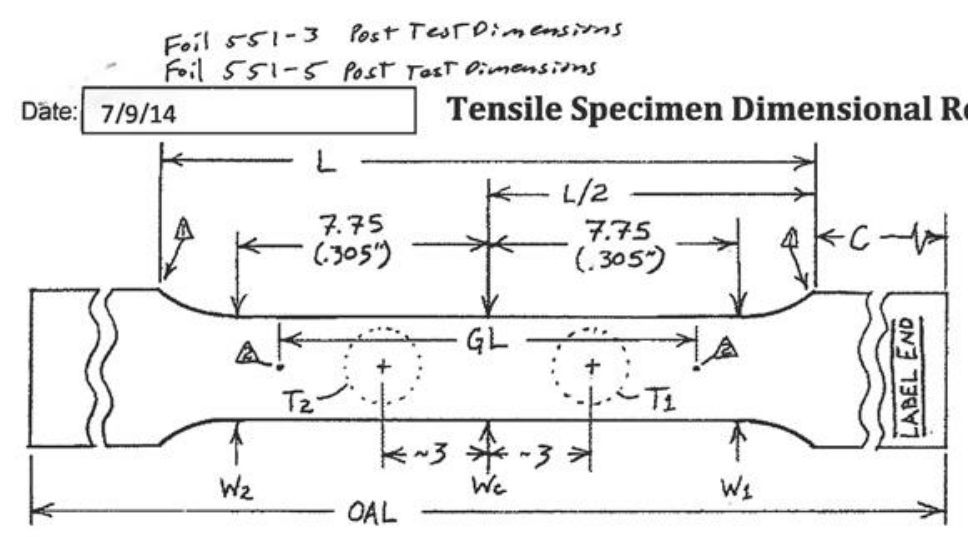

OAL measurement instrument ID: A678

GL measurement instrument ID: A678

W measurement instrument ID: $A 678$

T measurement instrument ID: $A 609$

Temperature instrument ID: A52393

Start of Measurements: $19.3 C^{\circ}$

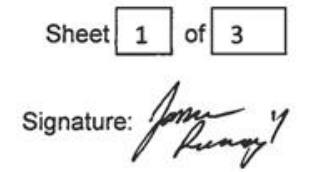

Expiration Date: 4/9/15

Expiration Date: $4 / 9 / 15$

Expiration Date: 4/9/15

Expiration Date: 11/13/14

Expiration Date: 6/23/15 End of Measurements: $19.0 C^{\circ}$

Delta 1. Reference positions for reduced section locations - corner at intersection of end tab and fillet. Locate center between corners (L/2) as zero position reference. It is not necessary to record dimension $\mathrm{L}$.

Delta 2. Conical indent marks for GL measurement are small, less than $0.1 \mathrm{~mm}$ typical. Measure indents center-to-center three times with optical comparator. Reset reference zero for each measurement (three independent measurements).

1. Locations to measure are indicated in the diagram. All dimensions in mm except as indicated by (in.). Record measurements to $0.001 \mathrm{~mm}$ excepting " $\mathrm{C}^{\text {" }}$ and

2. "OAL."

3. Record dimension "OAL" to $0.01 \mathrm{~mm}$ resolution. This can be measured with a caliper

4. Measure $W_{1}, W_{2}$, and $W_{c}$ two times. Reset width reference zero for each measurement (make two independent measurements at each location).

5. Measure thicknesses $T_{1}$ and $T_{2}$, twice at each location, approximately $3 \mathrm{~mm}$ either side of centerline. Measuring device contact surface should not cover the gage mark indent. Ball tip micrometer use is suggested for small contact area on indent face of specimen. Use minimum pressure needed to achieve consistent measurements.

6. Record room temperature before starting and after completion of each group of measurements.

\begin{tabular}{|c|c|c|c|c|c|c|c|c|c|c|c|c|c|c|c|}
\hline \multirow{2}{*}{$\begin{array}{c}\text { Specimen } \\
\text { ID }\end{array}$} & \multirow{2}{*}{$\begin{array}{l}\mathrm{OAL} \\
(\mathrm{mm}) \\
\end{array}$} & \multirow{2}{*}{$\begin{array}{c}\mathrm{C} \\
(\mathrm{mm}) \\
\end{array}$} & \multicolumn{2}{|c|}{$\mathrm{W}_{1}(\mathrm{~mm})$} & \multicolumn{2}{|c|}{$\mathrm{W}_{2}(\mathrm{~mm})$} & \multicolumn{2}{|c|}{$W_{c}(\mathrm{~mm})$} & \multicolumn{3}{|c|}{$\mathrm{GL}(\mathrm{mm})$} & \multicolumn{2}{|c|}{$\mathrm{T}_{1}(\mathrm{~mm})$} & \multicolumn{2}{|c|}{$T_{2}(\mathrm{~mm})$} \\
\hline & & & $\# 1$ & $\# 2$ & $\# 1$ & \#2 & $\# 1$ & $\# 2$ & $\# 1$ & $\# 2$ & $\# 3$ & $\# 1$ & \#2 & $\# 1$ & \#2 \\
\hline $551-3$ L29 & 51.552 & 15.442 & 3.168 & 3.168 & 3.156 & 3.156 & 3.123 & 3.123 & 13.394 & 13.397 & 13.393 & .370 & .371 & .371 & .372 \\
\hline $551-5 \mathrm{~L} 1$ & 52.211 & 15.544 & 3.126 & 3.126 & 3.130 & 3.129 & 2.879 & 2.879 & 14.082 & 14.084 & 14.085 & .499 & .500 & .487 & .487 \\
\hline L2 & 52.445 & 15.387 & 3.162 & 3.162 & 3.120 & 3.118 & 1.991 & 1.992 & 14.285 & 14.285 & 14.281 & .518 & .518 & .506 & .506 \\
\hline L3 & 52.387 & 15.416 & 3.148 & 3.148 & 3.131 & 3.132 & 2.916 & 2.195 & 14.239 & 14.240 & 14.238 & .520 & .520 & .502 & .502 \\
\hline L4 & 52.655 & 15.429 & 3.113 & 3.113 & 3.112 & 3.112 & 2.112 & 2.113 & 14.520 & 14.527 & 14.531 & .520 & .519 & .507 & .506 \\
\hline L5 & 51.351 & 15.417 & 3.185 & 3.186 & 3.190 & 3.190 & 3.150 & 3.149 & 13.292 & 13.299 & 13.293 & .498 & .498 & .504 & .504 \\
\hline L6 & 51.687 & 15.378 & 3.166 & 3.165 & 3.134 & 3.134 & 3.130 & 3.130 & 13.592 & 13.587 & 13.587 & .509 & .510 & .512 & .512 \\
\hline L10 & 55.825 & 15.466 & 3.026 & 3.025 & 2.986 & 2.986 & 2.768 & 2.767 & 17.307 & 17.293 & 17.292 & .424 & .424 & .428 & .428 \\
\hline
\end{tabular}


TEM-10300-1

03/01/2012

TECHNICAL EVALUATION

Page B22 of B35

Rev. 03

Title:

Elevated Temperature Tensile Tests on DU-10Mo Rolled Foils

TEV No.: 2194

Rev. No.:

2

Project No.:

25228

Date:

05/02/18

Date: $7 / 9 / 14$

Tensile Specimen Dimensional Record Sheet (continuation)

Sheet 2 of 3

Measurement Operator Name: James Reseigh

signature: Jame phenayl

\begin{tabular}{|c|c|c|c|c|c|c|c|c|c|c|c|c|c|c|c|}
\hline \multirow{2}{*}{$\begin{array}{c}\text { Specimen } \\
\text { ID }\end{array}$} & \multirow{2}{*}{$\begin{array}{l}\mathrm{OAL} \\
(\mathrm{mm})\end{array}$} & \multirow{2}{*}{$\begin{array}{c}\text { C } \\
(\mathrm{mm})\end{array}$} & \multicolumn{2}{|c|}{$\mathrm{W}_{1}(\mathrm{~mm})$} & \multicolumn{2}{|c|}{$\mathrm{W}_{2}(\mathrm{~mm})$} & \multicolumn{2}{|c|}{$W_{c}(\mathrm{~mm})$} & \multicolumn{3}{|c|}{$\mathrm{GL}(\mathrm{mm})$} & \multicolumn{2}{|c|}{$T_{1}(\mathrm{~mm})$} & \multicolumn{2}{|c|}{$\mathrm{T}_{2}(\mathrm{~mm})$} \\
\hline & & & $\# 1$ & \#2 & $\# 1$ & \#2 & $\# 1$ & $\# 2$ & $\# 1$ & $\# 2$ & $\# 3$ & $\# 1$ & $\# 2$ & $\# 1$ & \#2 \\
\hline L11 & 57.060 & 15.482 & 2.982 & 2.982 & 2.977 & 2.977 & 2.737 & 2.737 & 18.144 & 18.452 & 18.140 & .416 & .416 & .406 & .407 \\
\hline L12 & 56.576 & 15.432 & 3.044 & 3.044 & 2.907 & 2.907 & 2.762 & 2.762 & 17.789 & 17.785 & 17.776 & .430 & .430 & .409 & .409 \\
\hline T1 & 50.871 & 15.347 & 3.217 & 3.217 & 3.229 & 3.229 & 3.195 & 3.195 & 12.863 & 12.861 & 12.860 & .532 & .532 & .543 & .543 \\
\hline T2 & 50.909 & 15.340 & 3.175 & 3.174 & 3.172 & 3.172 & 3.156 & 3.156 & 12.864 & 12.865 & 12.867 & .545 & .545 & .544 & .545 \\
\hline T3 & 50.832 & 15.383 & 3.196 & 3.196 & 3.182 & 3.182 & 3.163 & 3.162 & 12.861 & 12.871 & 12.863 & .547 & .547 & .544 & .544 \\
\hline T4 & 51.066 & 15.380 & 3.184 & 3.183 & 3.188 & 3.189 & 3.161 & 3.161 & 12.946 & 12.941 & 12.941 & .537 & .537 & .537 & .537 \\
\hline T5 & 51.202 & 15.391 & 3.194 & 3.194 & 3.176 & 3.174 & 3.159 & 3.158 & 13.118 & 13.115 & 13.122 & .536 & .536 & .537 & .537 \\
\hline T6 & 51.440 & 15.375 & 3.183 & 3.183 & 3.181 & 3.181 & 3.162 & 3.161 & 13.359 & 13.360 & 13.359 & .537 & .537 & .530 & .530 \\
\hline T10 & 57.439 & 15.487 & 2.955 & 2.957 & 3.003 & 3.003 & 2.798 & 2.799 & 18.597 & 18.594 & 18.583 & .404 & .404 & .365 & .366 \\
\hline T11 & 57.278 & 15.431 & 2.993 & 2.992 & 3.006 & 3.006 & 2.882 & 2.883 & 18.527 & 18.523 & 18.531 & .396 & .396 & .415 & .415 \\
\hline T12 & 57.620 & 15.448 & 2.979 & 2.978 & 3.036 & 3.036 & 2.918 & 2.918 & 18.702 & 18.710 & 18.691 & .400 & .400 & .430 & .430 \\
\hline $551-3$ L1 & 51.680 & 15.138 & 3.159 & 3.159 & 3.158 & 3.157 & 2.965 & 2.966 & 13.830 & 13.833 & 13.835 & .358 & .358 & .357 & .357 \\
\hline L2 & 51.718 & 15.142 & 3.144 & 3.143 & 3.155 & 3.155 & 2.975 & 2.974 & 13.862 & 13.855 & 13.850 & .356 & .356 & .363 & .363 \\
\hline L3 & 51.710 & 15.156 & 3.171 & 3.171 & 3.156 & 3.156 & 2.990 & 2.990 & 13.845 & 13.842 & 13.843 & .361 & .361 & .369 & .369 \\
\hline L4 & 52.398 & 15.232 & 3.104 & 3.105 & 3.104 & 3.102 & 3.014 & 3.014 & 14.473 & 14.475 & 14.484 & .363 & .363 & .361 & .361 \\
\hline L5 & 52.419 & 15.138 & 3.049 & 3.049 & 3.115 & 3.115 & 3.059 & 3.058 & 14.343 & 14.340 & 14.342 & .362 & .362 & .370 & .370 \\
\hline L6 & 52.351 & 15.125 & 3.088 & 3.088 & 3.110 & 3.111 & 3.012 & 3.013 & 14.471 & 14.483 & 14.475 & .362 & .362 & .371 & .369 \\
\hline L7 & 51.083 & 15.100 & 3.170 & 3.169 & 3.163 & 3.164 & 3.117 & 3.117 & 13.203 & 13.198 & 13.206 & .380 & .380 & .384 & .384 \\
\hline L8 & 51.093 & 15.114 & 3.129 & 3.129 & 3.193 & 3.193 & 3.151 & 3.151 & 13.145 & 13.143 & 13.149 & .385 & .385 & .385 & .385 \\
\hline L9 & 51.409 & 15.107 & 3.180 & 3.179 & 3.079 & 3.079 & 3.129 & 3.130 & 13.106 & 13.110 & 13.114 & .384 & .384 & .384 & .384 \\
\hline
\end{tabular}

Measurement Notes (use back of sheet if needed): 
TEM-10300-1

03/01/2012

TECHNICAL EVALUATION

Page B23 of B35

Rev. 03

Title:

Elevated Temperature Tensile Tests on DU-10Mo Rolled Foils

TEV No.: 2194

Rev. No.:

2

Project No.:

25228

Date:

05/02/18

Date: $7 / 9 / 14$

Tensile Specimen Dimensional Record Sheet (continuation)

Sheet 3 of 3

Measurement Operator Name: James Reseigh

signature: forme thumat

\begin{tabular}{|c|c|c|c|c|c|c|c|c|c|c|c|c|c|c|c|}
\hline \multirow{2}{*}{$\begin{array}{c}\text { Specimen } \\
\text { ID }\end{array}$} & \multirow{2}{*}{$\begin{array}{l}\text { OAL } \\
(\mathrm{mm})\end{array}$} & \multirow{2}{*}{$\begin{array}{c}\mathrm{C} \\
(\mathrm{mm})\end{array}$} & \multicolumn{2}{|c|}{$W_{1}(\mathrm{~mm})$} & \multicolumn{2}{|c|}{$\mathrm{W}_{2}(\mathrm{~mm})$} & \multicolumn{2}{|c|}{$W_{C}(\mathrm{~mm})$} & \multicolumn{3}{|c|}{$\mathrm{GL}(\mathrm{mm})$} & \multicolumn{2}{|c|}{$T_{1}(\mathrm{~mm})$} & \multicolumn{2}{|c|}{$\mathrm{T}_{2}(\mathrm{~mm})$} \\
\hline & & & $\# 1$ & $\# 2$ & $\# 1$ & $\# 2$ & $\# 1$ & $\# 2$ & $\# 1$ & $\# 2$ & $\# 3$ & $\# 1$ & \#2 & $\# 1$ & \#2 \\
\hline L10 & 60.781 & 15.424 & 2.778 & 2.779 & 2.980 & 2.979 & 2.528 & 2.528 & 21.465 & 21.465 & 21.474 & .251 & .251 & .278 & .278 \\
\hline L11 & 59.039 & 15.300 & 2.834 & 2.833 & 2.995 & 2.993 & 2.357 & 2.356 & 20.294 & 20.289 & 20.286 & .266 & .265 & .290 & .293 \\
\hline L12 & 61.740 & 15.133 & 2.880 & 2.879 & 2.831 & 2.830 & 2.111 & 2.110 & 22.488 & 22.486 & 22.494 & .270 & .270 & .270 & .270 \\
\hline L13 & 51.338 & 15.105 & 3.155 & 3.155 & 3.156 & 3.157 & 3.088 & 3.089 & 13.457 & 13.452 & 13.456 & .373 & .373 & .375 & .375 \\
\hline $551-3 \mathrm{~T} 1$ & 51.613 & 15.078 & 3.151 & 3.150 & 3.148 & 3.148 & 3.080 & 3.079 & 13.628 & 13.624 & 13.626 & .376 & .375 & .385 & .384 \\
\hline T2 & 51.623 & 15.105 & 3.134 & 3.133 & 3.157 & 3.157 & 3.087 & 3.087 & 13.650 & 13.651 & 13.647 & .376 & .376 & .383 & .383 \\
\hline T3 & 51.568 & 15.085 & 3.128 & 3.128 & 3.159 & 3.159 & 3.089 & 3.088 & 13.673 & 13.673 & 13.672 & .380 & .380 & .383 & .384 \\
\hline T4 & 52.281 & 15.073 & 3.063 & 3.063 & 3.086 & 3.087 & 3.039 & 3.039 & 14.260 & 14.242 & 14.237 & .378 & .378 & .372 & .372 \\
\hline T5 & 52.813 & 15.076 & 3.099 & 3.098 & 3.029 & 3.029 & 2.959 & 2.959 & 14.778 & 14.767 & 14.769 & .372 & .372 & .369 & .369 \\
\hline T6 & 52.367 & 15.166 & 3.039 & 3.038 & 3.130 & 3.130 & 3.037 & 3.068 & 14.249 & 14.241 & 14.247 & .376 & .376 & .383 & .383 \\
\hline "T7 & See & Note & & & & & & & & & & & & & \\
\hline T8 & 51.337 & 15.038 & 3.191 & 3.191 & 3.199 & 3.199 & 3.116 & 3.115 & 13.301 & 13.314 & 13.307 & .388 & .388 & .391 & .391 \\
\hline T9 & 51.185 & 15.029 & 3.165 & 3.165 & 3.172 & 3.173 & 3.108 & 3.107 & 13.408 & 13.406 & 13.409 & .389 & .389 & .394 & .394 \\
\hline T10 & 60.966 & 15.119 & 2.952 & 2.952 & 2.842 & 2.843 & 2.587 & 2.589 & 21.592 & 21.597 & 21.589 & .267 & .267 & .254 & .254 \\
\hline T11 & 59.841 & 15.092 & 2.922 & 2.922 & 2.918 & 2.917 & 2.447 & 2.445 & 20.657 & 20.672 & 20.672 & .257 & .258 & .288 & .287 \\
\hline T12 & 60.510 & 15.070 & 2.902 & 2.902 & 2.922 & 2.922 & 2.631 & 2.631 & 21.206 & 21.203 & 21.195 & .254 & .254 & .291 & .291 \\
\hline T13 & 51.410 & 14.929 & 3.151 & 3.152 & 3.170 & 3.169 & 3.127 & 3.126 & 13.506 & 13.504 & 13.505 & .391 & .391 & .390 & .390 \\
\hline & & & & & & & & & & & & & & & \\
\hline & & & & & & & & & & & & & & & \\
\hline & & & & & & & & & & & & & & & \\
\hline
\end{tabular}

Measurement Notes (use back of sheet if needed): T7 Broke in 3 pieces not able to get accurate measurements without additional fixturing. 
Rev. 03

Title:

\section{Elevated Temperature Tensile Tests on DU-10Mo Rolled Foils}

TEV No.:

Rev. No.:

2

Project No:

25228

Date:

$05 / 02 / 18$

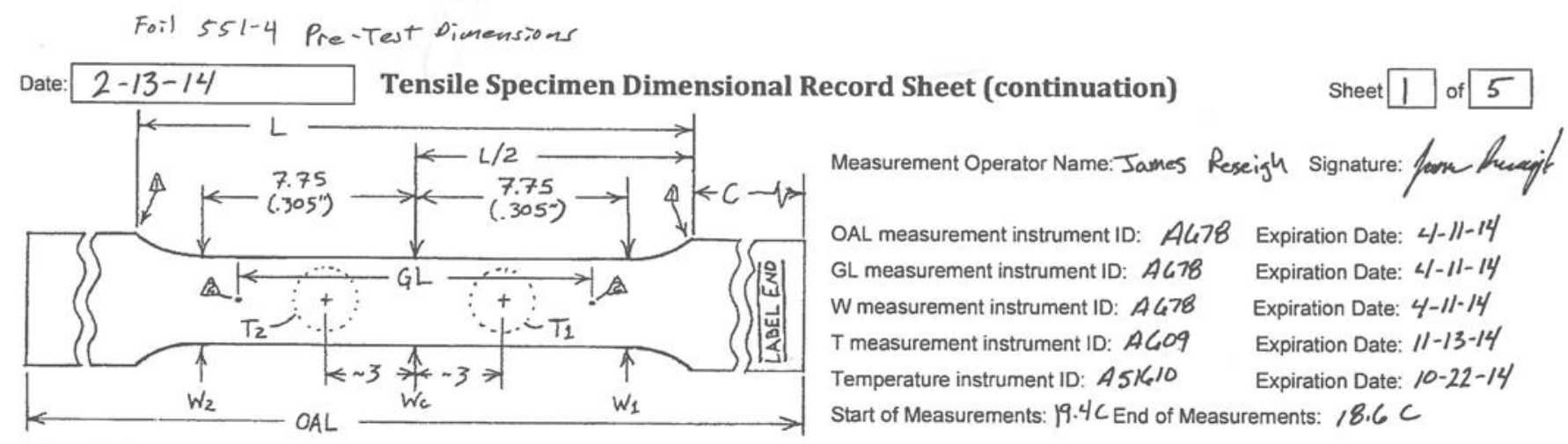

Delta 1. Reference positions for reduced section locations - corner at intersection of end tab and fillet. Locate center between corners (L/2) as zero position reference. It is not necessary to record dimension $L$.

Delta 2. Conical indent marks for GL measurement are small, less than $0.1 \mathrm{~mm}$ typical. Measure indents center-to-center three times with optical comparator. Reset reference zero for each measurement (three independent measurements).

. Locations to measure are indicated in the diagram. All dimensions in mm except as indicated by (in.). Record measurements to $0.001 \mathrm{~mm}$ excepting " $\mathrm{C}$ " and "OAL.

2. Record dimension " $\mathrm{C}$ " to $0.01 \mathrm{~mm}$ resolution.

3. Record dimension "OAL" to $0.01 \mathrm{~mm}$ resolution. This can be measured with a caliper.

4. Measure $W_{1}, W_{2}$, and $W_{c}$ two times. Reset width reference zero for each measurement (make two independent measurements at each location).

Measure thicknesses $\mathrm{T}_{\text {, and }} \mathrm{T}_{2}$, twice at each location, approximately $3 \mathrm{~mm}$ either side of centerline. Measuring device contact surface should not cover the gage mark indent. Ball tip micrometer use is suggested for small contact area on indent face of specimen. Use minimum pressure needed to achieve consistent

6. Reasurements.

\begin{tabular}{|c|c|c|c|c|c|c|c|c|c|c|c|c|c|c|c|}
\hline \multirow{2}{*}{$\begin{array}{c}\text { Specimen } \\
\text { ID }\end{array}$} & \multirow{2}{*}{$\begin{array}{l}\text { OAL } \\
(\mathrm{mm})\end{array}$} & \multirow{2}{*}{$\begin{array}{c}\mathrm{C} \\
(\mathrm{mm})\end{array}$} & \multicolumn{2}{|c|}{$W_{1}(\mathrm{~mm})$} & \multicolumn{2}{|c|}{$\mathrm{W}_{2}(\mathrm{~mm})$} & \multicolumn{2}{|c|}{$W_{c}(\mathrm{~mm})$} & \multicolumn{3}{|c|}{$\mathrm{GL}(\mathrm{mm})$} & \multicolumn{2}{|c|}{$T_{1}(\mathrm{~mm})$} & \multicolumn{2}{|c|}{$T_{2}(\mathrm{~mm})$} \\
\hline & & & $\# 1$ & \#2 & $\# 1$ & $\# 2$ & $\# 1$ & $\# 2$ & $\# 1$ & \#2 & $\# 3$ & $\# 1$ & $\# 2$ & $\# 1$ & $\# 2$ \\
\hline TI & 50,630 & 15.260 & 3.038 & 3.038 & 3.0415 & 3.045 & 3.009 & 3.008 & 12.763 & 12.751 & 12.754 & .389 & .389 & 388 & 388 \\
\hline$T 2$ & 50.593 & 15.369 & 3.198 & 3.198 & 3.198 & 3.197 & 3.171 & 3.172 & 12.766 & 12.772 & 12.770 & .389 & .388 & .389 & 389 \\
\hline T3 & 50.621 & 15.365 & 3.184 & 3.184 & 3.198 & 3.199 & 3.165 & 3.165 & 12.747 & 12.740 & 12.762 & .390 & .390 & .389 & 389 \\
\hline$T 4$ & 50.740 & 15.362 & 3.191 & 3.191 & 3.200 & 3.200 & 3.166 & 3.166 & 12.727 & 12.719 & 12.717 & .389 & 389 & .388 & 388 \\
\hline TS & 50.704 & 15.349 & 3.192 & 3.192 & 3.201 & 3.201 & 3.169 & 3.170 & 12.780 & 12.783 & 12.771 & .390 & .390 & .387 & .387 \\
\hline$T 6$ & 50.741 & 15.371 & 3.186 & 3.186 & 3.181 & 3.181 & 3.166 & 3.165 & 12.791 & 12.793 & 12.793 & .390 & .390 & .390 & .390 \\
\hline$T 7$ & 50.795 & 15.348 & 3.187 & 3.187 & 3.195 & 3.195 & 3.163 & 3.164 & 12.757 & 12.743 & $12.78 / 4$ & .390 & .390 & .388 & .388 \\
\hline TB & 50.827 & 15.340 & 3.196 & 3.195 & 3.191 & 3.191 & 3.761 & 3.161 & 12.758 & 12.748 & 12.745 & 390 & .390 & 389 & 389 \\
\hline
\end{tabular}


TEM-10300-1

03/01/2012

TECHNICAL EVALUATION

Page B25 of B35

Rev. 03

Title:

Elevated Temperature Tensile Tests on DU-10Mo Rolled Foils

TEV No.:

2194

Rev. No.:

2

Project No.:

25228

Date:

$05 / 02 / 18$

Date: $2-13-14$

Tensile Specimen Dimensional Record Sheet (continuation)

Sheet 2 of 5

Measurement Operator Name: James Reseigh signature: faim thumith

\begin{tabular}{|c|c|c|c|c|c|c|c|c|c|c|c|c|c|c|c|}
\hline ecimen & $\overline{O A L}$ & C & $W_{1}($ & & & & & & & $\mathrm{GL}(\mathrm{mm}$ & & & & & nm) \\
\hline & $(\mathrm{mm})$ & $(\mathrm{mm})$ & $\pi+1$ & \pm 2 & $\# 1$ & $\# 2$ & - & $\# 2$ & $\# 1$ & $\# 2$ & $\# 3$ & $\# 1$ & $\# 2$ & $\# 1$ & $\# 2$ \\
\hline-9 & 50.870 & 15.355 & 3.184 & 3.184 & 3.179 & 3.179 & 3.154 & 3.154 & 12.779 & 12.781 & 12.783 & .388 & .388 & .389 & 389 \\
\hline TIO & 50.875 & 15.336 & 3.184 & 3.184 & 3.192 & 3.191 & 3.152 & 3.152 & 12.785 & 12.774 & 12.777 & .391 & .391 & .390 & .391 \\
\hline TII & 51.019 & 15.501 & 3.173 & 3.173 & 3.185 & 3.185 & 3.157 & 3.157 & 12.722 & 12.718 & 12.717 & .391 & .391 & .391 & .391 \\
\hline$T 12$ & 51.060 & 15.497 & 3.182 & 3.183 & .178 & 3.178 & 3.151 & 3.151 & 12.712 & 12.711 & 12.717 & .391 & .391 & .389 & .389 \\
\hline$T 13$ & 51.113 & 15.497 & 3.175 & 3.174 & .175 & 3.175 & 3.156 & 3.156 & 12.727 & 12.722 & 12.710 & .391 & .391 & .391 & .391 \\
\hline$T 14$ & 51.310 & 15.510 & 3.198 & 3.198 & 3.185 & 3.186 & 3.182 & 3.183 & 12.715 & 12.717 & 12.709 & .390 & .390 & .390 & .390 \\
\hline$T 15$ & 51.152 & 15.488 & 3.184 & 3.183 & .183 & 3.183 & 3.140 & 3.160 & 12.724 & 12.715 & 12.713 & .390 & .390 & .590 & .390 \\
\hline THe & 51.152 & 15.494 & 3.187 & 3.186 & 3.186 & 3.185 & 3.160 & 3.161 & 12.714 & 12.709 & 12.705 & .392 & .391 & .391 & .390 \\
\hline$T I I$ & 51.170 & $15.4 / 92$ & 3.182 & 3.183 & 3.174 & 3.174 & 3.163 & 3.165 & 12.450 & 12.651 & 12.653 & .391 & .391 & .390 & .390 \\
\hline$T 18$ & 51.169 & 15.495 & 3.178 & 3.178 & 3.183 & 3.183 & 3.164 & 3.164 & 12.709 & 12.718 & 12.719 & .391 & .391 & .590 & .390 \\
\hline$T 19$ & 5.180 & 15.522 & 3.167 & 3.167 & 3.155 & 3.156 & 3.155 & 3.156 & 12.691 & 12.690 & 12.694 & .390 & .390 & .389 & .389 \\
\hline$T 20$ & 53.360 & 15.362 & 3.186 & 3.186 & 3.185 & 3.185 & 3.163 & 3.163 & 12.700 & 12.713 & 12.708 & .393 & .393 & .393 & .393 \\
\hline$T 21$ & 53.340 & 15.347 & 3.189 & 3.190 & 182 & 3.182 & 3.156 & 3.156 & 12.681 & 12.489 & 12.686 & .394 & .394 & .391 & .391 \\
\hline$T 22$ & 53.311 & 15.334 & 3.178 & 3.176 & 3.173 & 3.173 & 3.159 & 3.159 & 12.783 & 12.790 & 12.785 & .392 & .393 & .391 & .393 \\
\hline+23 & 53.326 & 15.319 & 3.170 & 3.170 & 3.170 & 3.171 & 3.156 & 3.157 & 12.682 & 12.670 & 12.684 & .393 & .394 & .393 & .393 \\
\hline$T 24$ & 53.2410 & 15.327 & 3.174 & 3.174 & 3.178 & 3.178 & 3.162 & 3.161 & 12.403 & 12.597 & 12.598 & .391 & .391 & .393 & .393 \\
\hline$T 25$ & 53.320 & 15.308 & 3.176 & 3.176 & 3.174 & 3.175 & 3.156 & 3.155 & 12.728 & 12.733 & 12.735 & .394 & .393 & .393 & .393 \\
\hline$T 26$ & 53.200 & 15.303 & 3.148 & 169 & 3.174 & 3.174 & 3.150 & 3.151 & 12.737 & 12.729 & 12.726 & .392 & .391 & .392 & .392 \\
\hline T27 & 53.235 & 15.278 & 3.179 & 3.179 & 3.173 & 3.173 & 3.158 & 3.158 & 12.672 & 12.678 & 12.671 & .393 & .393 & .392 & .392 \\
\hline$T^{28}$ & 53.240 & 15.283 & 3.171 & 3.171 & 3.178 & 3.179 & 3.148 & 3.148 & 12.839 & 12.833 & 12.831 & .393 & .393 & .391 & .391 \\
\hline
\end{tabular}

Measurement Notes (use back of sheet if needed): 
TEM-10300-1

03/01/2012

TECHNICAL EVALUATION

Page B26 of B35

Rev. 03

Title:

Elevated Temperature Tensile Tests on DU-10Mo Rolled Foils

TEV No.:

2194

Rev. No.:

2

Project No.:

25228

Date:

$05 / 02 / 18$

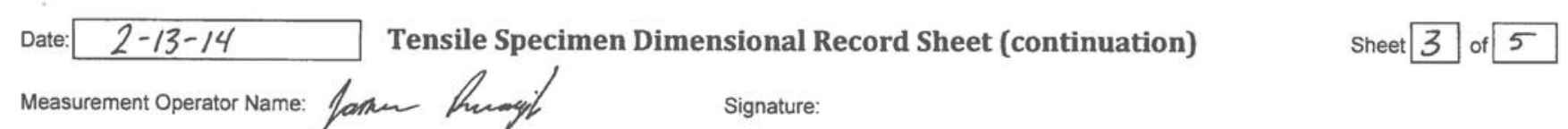

\begin{tabular}{|c|c|c|c|c|c|c|c|c|c|c|c|c|c|c|c|}
\hline \multirow{2}{*}{$\begin{array}{c}\text { Specimen } \\
\text { ID }\end{array}$} & \multirow{2}{*}{$\begin{array}{l}\mathrm{OAL} \\
(\mathrm{mm}) \\
\end{array}$} & \multirow{2}{*}{$\begin{array}{c}\mathrm{C} \\
(\mathrm{mm}) \\
\end{array}$} & \multicolumn{2}{|c|}{$\mathrm{W}_{1}(\mathrm{~mm})$} & \multicolumn{2}{|c|}{$\mathrm{W}_{2}(\mathrm{~mm})$} & \multicolumn{2}{|c|}{$W_{c}(\mathrm{~mm})$} & \multicolumn{3}{|c|}{$\mathrm{GL}(\mathrm{mm})$} & \multicolumn{2}{|c|}{$\mathrm{T}_{1}(\mathrm{~mm})$} & \multicolumn{2}{|c|}{$\mathrm{T}_{2}(\mathrm{~mm})$} \\
\hline & & & $\# 1$ & $\# 2$ & $\# 1$ & $\# 2$ & $\# 1$ & $\# 2$ & $\# 1$ & $\# 2$ & $\# 3$ & $\# 1$ & $\# 2$ & $\# 1$ & $\# 2$ \\
\hline$T 29$ & 53.195 & 15.278 & 3.183 & 3.183 & 3.174 & 3.175 & 3.160 & 3.160 & 12.681 & 12.485 & 12.679 & .394 & .393 & .392 & .392 \\
\hline T30 & 53.251 & 15.241 & 3.169 & 3.170 & 3.173 & 3.173 & 3.153 & 3.153 & 12.677 & 12.679 & 12.675 & .393 & .393 & .393 & .393 \\
\hline T31 & 53.147 & 15.247 & 3.177 & 3.176 & 3.176 & 3.176 & $3.14 / 9$ & 3.149 & 12.751 & 12.742 & 12.741 & .393 & .393 & .393 & .393 \\
\hline$T 32$ & 53.220 & 15.235 & 3.167 & 3.167 & 3.173 & 3.173 & 3.1417 & $3.14 / 6$ & 12.773 & 12.775 & 12.782 & .392 & .392 & .390 & .390 \\
\hline T33 & 52.828 & 15.240 & 3.177 & 3.176 & 3.176 & 3.176 & 3.170 & 3.170 & 12.741 & 12.736 & 12.725 & 390 & .390 & .390 & .391 \\
\hline T34 & 53.059 & 15.240 & 3.170 & 3.169 & 3.186 & 3.185 & 3.159 & 3.158 & 12.737 & 12.729 & 12.723 & .393 & .393 & .392 & .393 \\
\hline T35 & 53.143 & 15.217 & 3.169 & 3.169 & 3.182 & 3.182 & 3.160 & 3.160 & 12.645 & 12649 & 12.6417 & .391 & .391 & .391 & .391 \\
\hline T36 & 53.057 & 15.218 & 3.176 & 3.175 & 3.184 & 3.184 & 3.1421 & 3.144 & 12.466 & 12.670 & 12.467 & .392 & .392 & .393 & .392 \\
\hline T37 & 53.080 & 15.206 & 3.171 & 3.172 & 3.186 & 3.187 & 3.158 & 3.158 & 12.831 & 12.827 & 12.822 & .392 & 392 & .391 & .392 \\
\hline$T 38$ & 53.146 & 15.109 & 3.150 & 3.150 & 3.149 & 3.149 & 3.146 & 3.167 & 12.731 & 12.732 & 12.745 & .393 & .393 & .392 & .392 \\
\hline$L 1$ & 50.244 & 15.412 & 3.208 & 3.207 & 3.206 & 3.206 & 3.168 & 3.167 & 12 & 12.769 & 12,760 & .373 & .373 & .373 & .372 \\
\hline$\angle 2$ & 50.211 & 15.398 & 3.185 & 3.184 & 3.186 & 3.186 & 3.171 & 3.171 & $12 . x 61$ & 12.755 & $12 . x 2$ & .377 & .377 & .378 & .378 \\
\hline 13 & 50.185 & 15.404 & 3.181 & 3.181 & 3.176 & 3.176 & 3.160 & 3.160 & $12: 756$ & 12.758 & 12.740 & .381 & .381 & .381 & .382 \\
\hline$L 4$ & 50.183 & 15.394 & 3.176 & 3.177 & 3.177 & 3.178 & 3.151 & 3.152 & 12,784 & 12.770 & 12.775 & .386 & .386 & .386 & .386 \\
\hline$\angle 5$ & 50.180 & 15.417 & 3.181 & 3.181 & 3.191 & 3.190 & 3.152 & 5.151 & 12.839 & 12.854 & 12.859 & .386 & .386 & .387 & .386 \\
\hline$\angle 6$ & 50.179 & 15.4108 & 3.180 & 3.180 & 3.172 & 3.172 & 3.155 & 3.155 & 12.738 & 12.727 & 12.723 & .389 & .389 & .389 & .389 \\
\hline$\llcorner 7$ & 50.180 & 15.401 & 3.182 & 3.182 & 3.182 & 3.183 & 3.154 & 3.154 & 12.702 & 12.696 & 12.694 & .388 &, 388 & .390 & .390 \\
\hline L: & 50.182 & 15.399 & 3.188 & 3.188 & 3.185 & 3.185 & 3.160 & 3.159 & 12.739 & 12.744 & 12.7419 & 388 & .388 & .390 & .390 \\
\hline 19 & 50.181 & 15,385 & 3.180 & 3.180 & 3.177 & 3.177 & 3.155 & 3.155 & 12.712 & 12.704 & 12.713 & .386 & 386 & .386 & .386 \\
\hline
\end{tabular}

Measurement Notes (use back of sheet if needed):

T38 Has a raised section in the middle of speciften 
TEM-10300-1

03/01/2012

TECHNICAL EVALUATION

Page B27 of B35

Rev. 03

Title:

Elevated Temperature Tensile Tests on DU-10Mo Rolled Foils

TEV No.:

2194

Rev. No.:

2

Project No.:

25228

Date:

$05 / 02 / 18$

Date: $2-13-14$

Sheet 4 of 5

Measurement Operator Name: James Reseigh

Signature:

fare theracit

\begin{tabular}{|c|c|c|c|c|c|c|c|c|c|c|c|c|c|c|c|}
\hline \multirow{2}{*}{$\begin{array}{c}\begin{array}{c}\text { Specimen } \\
\text { ID }\end{array} \\
\end{array}$} & \multirow{2}{*}{$\begin{array}{l}\mathrm{OAL} \\
(\mathrm{mm}) \\
\end{array}$} & \multirow{2}{*}{$\begin{array}{c}\mathrm{C} \\
(\mathrm{mm}) \\
\end{array}$} & \multicolumn{2}{|c|}{$\overline{W_{1}}$} & \multicolumn{2}{|c|}{$\mathrm{W}_{2}(\mathrm{~mm})$} & \multicolumn{2}{|r|}{ m) } & \multicolumn{3}{|c|}{$\mathrm{GL}(\mathrm{mm})$} & \multicolumn{2}{|c|}{$T_{1}(\mathrm{~mm})$} & \multicolumn{2}{|c|}{$T_{2}(\mathrm{~mm})$} \\
\hline & & & $\# 1$ & $\# 2$ & $\# 1$ & $\# 2$ & $\# 1$ & 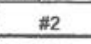 & $\# 1$ & $\# 2$ & $\# 3$ & $\# 1$ & $\# 2$ & $\# 1$ & $\# 2$ \\
\hline$\angle 10$ & 50.192 & 15.403 & 3.191 & 3.191 & 3.178 & 3.178 & 3.154 & 3.15 .3 & 12.716 & 12.719 & 12.715 & .386 & .386 & 387 & .387 \\
\hline 411 & 56.195 & 15.405 & 3.190 & 3.190 & 3.181 & 3.181 & 3.159 & 3.158 & 12.731 & 12.729 & 12.732 & .384 & .384 & 386 & .386 \\
\hline$L 12$ & 50.196 & 15.403 & 3.190 & 3.190 & 3.181 & 3.180 & 3.172 & 3.172 & 12.709 & 12.709 & 12.714 & .383 & .383 & 382 & 382 \\
\hline$L 13$ & 50.197 & 15.412 & 3.186 & 3.186 & 3.185 & 3.185 & 3.171 & 3.170 & 12794 & 12.798 & 12.791 & .377 & .377 & .378 & .378 \\
\hline L.14 & 50.201 & 15.394 & 3.189 & 3.189 & 3.187 & 3.188 & 3.161 & 3.161 & 12.775 & 12.764 & 12.769 & .370 & .370 & .369 & .369 \\
\hline$\angle 15$ & 59.888 & 15,385 & 3.203 & 3.204 & 3.193 & 3.192 & 3.161 & 3.161 & 12.813 & 12807 & 12.797 & .387 & .387 & .387 & 387 \\
\hline$\angle 16$ & 50.877 & 15.376 & 3.1218 & 3.1419 & 3.139 & 3.140 & 3.120 & 3.121 & 12.700 & 12.701 & 12.700 & .384 & .384 & .383 & .383 \\
\hline 417 & 50.877 & 5.420 & 3.156 & 3.156 & 3.1411 & $3.1 / 10$ & 3.125 & 3.124 & 12,464 & 12.651 & 12.44 & .378 & .378 & .378 & .378 \\
\hline$\angle 17 \mathrm{~A}$ & 50.857 & 15.625 & 3.182 & 3.181 & 3.180 & 3.180 & 3.161 & 3.161 & 12.841 & 12.834 & 12.827 & .374 & .374 & .374 & 374 \\
\hline$L 18$ & 50.461 & 15.417 & 3.190 & 3.191 & 3.207 & 3.206 & 3.171 & 3.172 & 12.746 & 12.745 & 12.736 & .386 & .386 & .386 & 386 \\
\hline 419 & 50.456 & 15.397 & 3.189 & 3.189 & 3.193 & 3.193 & 3.172 & 3.172 & 12.718 & 12.723 & 12.727 & .385 & .385 & .386 & .385 \\
\hline$\angle 20$ & 50.465 & 15.399 & 3.190 & 3.190 & 3.192 & 3.193 & 3.158 & 3.157 & 12.840 & 12.848 & 12.843 & 377 & .377 & .379 & .379 \\
\hline L21 & 50.460 & 15,382 & 3.181 & 3.180 & 3.197 & 3.196 & 3.153 & 3.153 & 12605 & 12.599 & 12,598 & .372 & .372 & 373 & 373 \\
\hline L22 & 50.587 & 15.539 & 3.201 & 3.202 & 3.191 & 3.191 & 3.169 & 3.168 & 12.676 & 12.667 & 12.670 & .374 & 374 & .374 & 374 \\
\hline 2.23 & 50.578 & 15.588 & 3.186 & 3.186 & 3.187 & 3.187 & 3.159 & 3.159 & 12.800 & 12.788 & 12.787 & 382 & 382 & 382 & .382 \\
\hline L24 & 50.556 & 15.578 & 3.186 & 3.186 & 3.181 & 3.181 & 3.161 & 3.161 & 12.781 & 12.792 & 12781 & .385 & 384 & .386 & .385 \\
\hline$\angle 25$ & 50.531 & 15,6412 & 3.174 & 3.173 & 3.178 & 3,179 & 3.154 & 3.154 & 12.729 & 12.730 & 12.732 & 388 & .388 & .390 & .391 \\
\hline$\angle 26$ & 50.729 & 15.391 & 3.184 & 3.184 & 3.187 & 3.187 & 3.163 & 3.163 & 12.757 & 12.751 & 12.749 & .372 & .373 & 372 & 374 \\
\hline$\angle 27$ & 50.721 & 15.4104 & 3.188 & 3.188 & 3.184 & 3.184 & 3.163 & 3.163 & 12.727 & 12.723 & 2.739 & .383 & .384 & .384 & 384 \\
\hline 128 & 50.700 & 15.389 & 3.192 & 3.191 & 3.193 & 3.193 & 3.163 & 3.161 & 12.735 & 12.744 & 12,748 & 385 &, 385 & .385 & .385 \\
\hline
\end{tabular}

Measurement Notes (use back of sheet if needed):

L25 Has one side that is almost elat, very little cut out. 
TEM-10300-1

03/01/2012

TECHNICAL EVALUATION

Page B28 of B35

Rev. 03

Title:

Elevated Temperature Tensile Tests on DU-10Mo Rolled Foils

TEV No.:

2194

Rev. No.:

2

Project No.:

25228

Date:

05/02/18

Date: Tensile Specimen Dimensional Record Sheet (continuation)

Sheet 5 of 5

Measurement Operator Name: James Reseigh

signature: Haren freceryi/

\begin{tabular}{|c|c|c|c|c|c|c|c|c|c|c|c|c|c|c|c|}
\hline \multirow{2}{*}{$\begin{array}{c}\text { Specimen } \\
\text { ID }\end{array}$} & \multirow{2}{*}{$\begin{array}{l}\text { OAL } \\
(\mathrm{mm}) \\
\end{array}$} & \multirow{2}{*}{$\frac{\mathrm{C}}{(\mathrm{mm})}$} & \multicolumn{2}{|c|}{$\mathrm{W}_{1}(\mathrm{~mm})$} & \multicolumn{2}{|c|}{$W_{2}(\mathrm{~mm})$} & \multicolumn{2}{|c|}{$\mathrm{W}_{c}(\mathrm{~mm})$} & \multicolumn{3}{|c|}{$\mathrm{GL}(\mathrm{mm})$} & \multicolumn{2}{|c|}{$T_{1}(\mathrm{~mm})$} & \multicolumn{2}{|c|}{$T_{2}(\mathrm{~mm})$} \\
\hline & & & $\# 1$ & $\# 2$ & $\# 1$ & $\# 2$ & $\# 1$ & \#2 & $\# 1$ & $\# 2$ & $\# 3$ & $\# 1$ & $\# 2$ & $\# 1$ & $\# 2$ \\
\hline$\angle 29$ & 50.677 & 15.368 & 3.192 & 3.193 & 3.187 & 3.187 & 3.158 & 3.158 & 12.722 & 12.727 & 12.728 & 389 & .389 & .390 & .389 \\
\hline$* \angle 29$ & 50.477 & 15.267 & 3.188 & 3.187 & 3.193 & 3.192 & 3.166 & 3.166 & 12.762 & 12.757 & 12.753 & .373 & .373 & .374 & .374 \\
\hline$\angle 30$ & 50.492 & 15.271 & 3.190 & 3.189 & 3.184 & 3.184 & 3.157 & 3.157 & 12.757 & 12.756 & 12.750 & .382 & .382 & .383 & .383 \\
\hline$\angle 31$ & 50.470 & 15.223 & 3.177 & 3.177 & 3.192 & 3.191 & 3.157 & 3.157 & 12.738 & 12.741 & 12.738 & .386 & .386 & .387 & .387 \\
\hline+32 & 50.463 & 15.221 & $3+190$ & 3.189 & 3.174 & 3.76 & 3.156 & 3.157 & 12.472 & 12.472 & 22.468 & .390 & .390 & .390 & .390 \\
\hline$L 33$ & 50.486 & 15.204 & 3.191 & 3.190 & 3.186 & 3.186 & 3.159 & 3.160 & 12.732 & 12.723 & 12.730 & .392 & .392 & 390 & .391 \\
\hline$L 34$ & 50.583 & 15.186 & 3.188 & 3.189 & 3.184 & 3.183 & 3.161 & 3.161 & 12.757 & 12.756 & 12.754 & .392 & .392 & .392 & .392 \\
\hline$\angle 35$ & 50.589 & 15.160 & 3.187 & 3.187 & 3.177 & 3.178 & 3.153 & 3.154 & 12.744 & 12.740 & 12.757 & .394 & .394 & .395 & .395 \\
\hline 4.36 & 50.592 & 15.180 & 3.181 & 3.182 & 3.173 & 3.173 & 3.160 & 3.160 & 12.736 & 12.738 & 12,739 & .3941 & .394 & .395 & .395 \\
\hline$L 37$ & 50.596 & 15.167 & 3.181 & 3.179 & 3.182 & 3.182 & 3.166 & 3.165 & 12.703 & 12.711 & 12.710 & .394 & .394 & .394 & .394 \\
\hline$L 38$ & 50601 & 15.145 & 3.187 & 3.185 & 3.187 & 3.187 & 3.162 & 3.161 & 12.888 & 12.894 & 12.892 & .393 & .393 & .393 & .393 \\
\hline 439 & 50.596 & 15.128 & 3.062 & 3.061 & 3.063 & 3.063 & 3.037 & 3.038 & 12.717 & 12.718 & 12.784 & .389 & .389 & .389 & .389 \\
\hline$\angle 40$ & 50593 & 15.115 & 3.078 & 3.078 & 3.067 & 3.066 & 3.044 & 3.045 & 12.822 & 12.828 & 12.820 & .387 & .387 & .387 & .387 \\
\hline$\angle 41$ & 50.608 & 15.226 & 3.082 & 3.082 & 3.083 & 3.084 & 3.050 & 3.050 & 12.736 & 12.740 & 12.746 & .384 & .384 & .384 & .384 \\
\hline$L 4 / 2$ & 50.672 & 15.219 & 3.100 & 3.100 & 3.095 & 3.094 & 3.084 & 3.085 & 12.689 & 12.690 & 12.690 & .375 & .375 & .376 & 376 \\
\hline$\angle 32$ & 50.463 & 15.221 & 3.190 & 3.189 & 3.176 & 3.176 & 3.156 & 3.157 & $12.74 / 2$ & 12.742 & $12.76 B$ & .390 & 390 & .390 & .390 \\
\hline & & & & & & & & & & & & & & & \\
\hline & & & & & & & & & & & & & & & \\
\hline & & & & & & & & & & & & & & & \\
\hline
\end{tabular}

$2 L 29$ 's * from Bag with unique 70 A-551-4-1-L6 
Rev. 03

Title:

TEV No.: 2194

Rev. No.:

2

Project No.:

25228

Date:

$05 / 02 / 18$

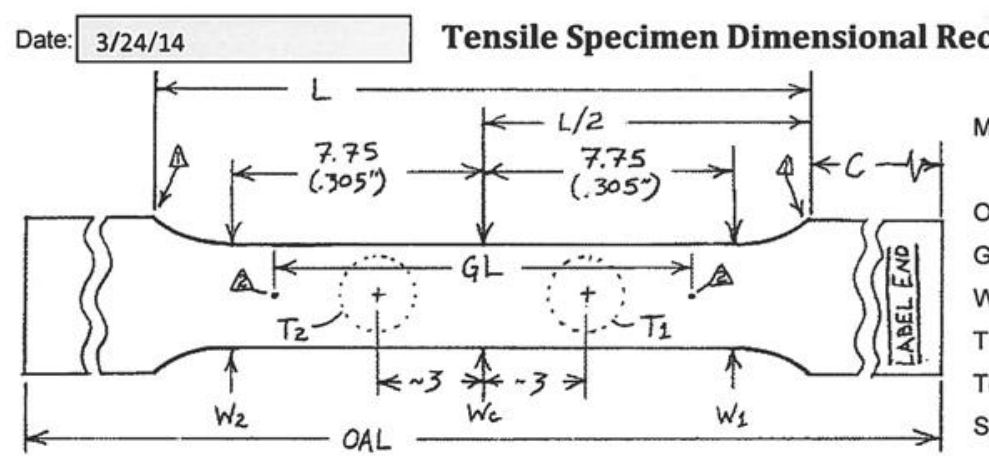

cord Sheet (continuation)

Measurement Operator Name: James Reseigh

OAL measurement instrument ID: A678

GL measurement instrument ID: A678

W measurement instrument ID: A678

T measurement instrument ID: A609

Temperature instrument ID: A51610

Start of Measurements: $18.6^{\circ} \mathrm{C}$

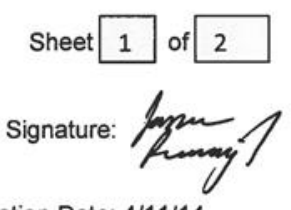

Expiration Date: 4/11/14

Expiration Date: 4/11/14

Expiration Date: $4 / 11 / 14$

Expiration Date: 11/13/14

Expiration Date: 10/22/14

End of Measurements: $19.5^{\circ} \mathrm{C}$

Delta 1. Reference positions for reduced section locations - corner at intersection of end tab and fillet. Locate center between corners (L/2) as zero position reference. It is not necessary to record dimension L.

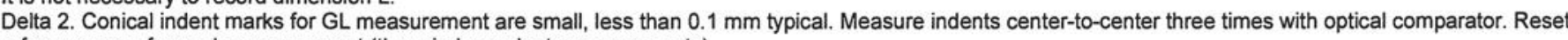
measurement (three independent measurements).

1. Locations to measure are indicated in the diagram. All dimensions in mm except as indicated by (in.). Record measurements to $0.001 \mathrm{~mm}$ excepting " $\mathrm{C}$ " and

2. Record dimension " $\mathrm{C}$ " to $0.01 \mathrm{~mm}$ resolution.

3. Record dimension "OAL" to $0.01 \mathrm{~mm}$ resolution. This can be measured with a caliper.

(make two independent measurements at each location).

5. Measure thicknesses $T_{1}$ and $T_{2}$, twice at each location, approximately $3 \mathrm{~mm}$ either side of centerline. Measuring device contact surface should not cover the gage mark indent. Ball tip micrometer use is suggested for small contact area on indent face of specimen. Use minimum pressure needed to achieve consistent measurements.

temperature before starting and after completion of each group of measurements.

\begin{tabular}{|c|c|c|c|c|c|c|c|c|c|c|c|c|c|c|c|}
\hline \multirow{2}{*}{$\begin{array}{l}\text { Specimen } \\
\text { ID }\end{array}$} & \multirow{2}{*}{$\frac{\mathrm{OAL}}{(\mathrm{mm})}$} & \multirow{2}{*}{$\frac{\mathrm{C}}{(\mathrm{mm})}$} & \multicolumn{2}{|c|}{$\mathrm{W}_{1}(\mathrm{~mm})$} & \multicolumn{2}{|c|}{$\mathrm{W}_{2}(\mathrm{~mm})$} & \multicolumn{2}{|c|}{$W_{c}(\mathrm{~mm})$} & \multicolumn{3}{|c|}{$\mathrm{GL}(\mathrm{mm})$} & \multicolumn{2}{|c|}{$T_{1}(\mathrm{~mm})$} & \multicolumn{2}{|c|}{$\mathrm{T}_{2}(\mathrm{~mm})$} \\
\hline & & & $\# 1$ & $\# 2$ & $\# 1$ & $\# 2$ & $\# 1$ & $\# 2$ & $\# 1$ & \#2 & $\# 3$ & $\# 1$ & \#2 & $\# 1$ & \#2 \\
\hline L1 & 51.098 & 15.433 & 3.172 & 3.172 & 3.176 & 3.176 & 3.063 & 3.063 & 13.560 & 13.558 & 13.545 & .366 & .366 & .371 & .371 \\
\hline L2 & 51.049 & 15.419 & 3.170 & 3.171 & 3.156 & 3.155 & 3.091 & 3.091 & 13.485 & 13.489 & 13.493 & .369 & .369 & .374 & .374 \\
\hline L3 & 50.990 & 15.411 & 3.158 & 3.159 & 3.160 & 3.159 & 3.079 & 3.078 & 13.482 & 13.478 & 13.479 & .375 & .375 & .381 & .381 \\
\hline L4 & 50.598 & 15.413 & 3.172 & 3.172 & 3.171 & 3.172 & 3.127 & 3.127 & 13.151 & 13.147 & 13.147 & .383 & .383 & .385 & .384 \\
\hline L5 & 50.582 & 15.370 & 3.165 & 3.165 & 3.173 & 3.173 & 3.099 & 3.100 & 13.222 & 13.217 & 13.216 & .386 & .386 & .387 & .386 \\
\hline L6 & 50.597 & 15.417 & \begin{tabular}{|l|}
3.178 \\
\end{tabular} & 3.177 & 3.165 & 3.165 & 3.076 & 3.077 & 13.101 & 13.103 & 13.112 & .388 & .388 & .387 & .387 \\
\hline *L10 & 57.920 & 15.684 & 3.011 & 3.012 & 2.963 & 2.963 & 2.580 & 2.581 & 19.161 & 19.163 & 19.159 & .281 & .281 & .283 & .283 \\
\hline "L11 & 57.091 & 15.627 & 3.024 & 3.025 & 3.004 & 3.005 & 2.769 & 2.769 & \begin{tabular}{|l|}
18.329 \\
\end{tabular} & 18.315 & 18.300 & .304 & .304 & .293 & .293 \\
\hline
\end{tabular}


Rev. 03

Elevated Temperature Tensile Tests on DU-10Mo Rolled Foils

Title:

\section{4}

Rev. No.:

2

Project No.:

25228

Date:

$05 / 02 / 18$

Date: $3 / 24 / 14$

Tensile Specimen Dimensional Record Sheet (continuation)

Measurement Operator Name: James Reseigh

Sheet 2 of 2

signature: Jamen thunefi!

\begin{tabular}{|c|c|c|c|c|c|c|c|c|c|c|c|c|c|c|c|}
\hline \multirow{2}{*}{$\begin{array}{c}\text { Specimen } \\
\text { ID }\end{array}$} & \multirow{2}{*}{$\begin{array}{l}\text { OAL } \\
(\mathrm{mm})\end{array}$} & \multirow{2}{*}{$\begin{array}{c}\mathrm{C} \\
(\mathrm{mm})\end{array}$} & \multicolumn{2}{|c|}{$\mathrm{W}_{1}(\mathrm{~mm})$} & \multicolumn{2}{|c|}{$\mathrm{W}_{2}(\mathrm{~mm})$} & \multicolumn{2}{|c|}{$W_{c}(\mathrm{~mm})$} & \multicolumn{3}{|c|}{$\mathrm{GL}(\mathrm{mm})$} & \multicolumn{2}{|c|}{$T_{1}(\mathrm{~mm})$} & \multicolumn{2}{|c|}{$\mathrm{T}_{2}(\mathrm{~mm})$} \\
\hline & & & $\# 1$ & $\# 2$ & $\# 1$ & $\# 2$ & $\# 1$ & $\# 2$ & $\# 1$ & $\# 2$ & $\# 3$ & $\# 1$ & \#2 & $\# 1$ & \#2 \\
\hline "L12 & 59.436 & 15.593 & 3.022 & 3.020 & 2.967 & 2.968 & 2.608 & 2.608 & 20.226 & 20.257 & 20.274 & .292 & .292 & .278 & .278 \\
\hline "L13 & 57.023 & 15.651 & 2.977 & 2.978 & 3.080 & 3.080 & 2.644 & 2.645 & 18.361 & 18.338 & 18.351 & .299 & .299 & .306 & .306 \\
\hline T1 & 50.612 & 15.312 & 3.025 & 3.026 & 3.035 & 3.035 & 2.991 & 2.991 & 13.038 & 13.028 & 13.038 & .386 & .386 & .385 & .384 \\
\hline T2 & 50.809 & 15.375 & 3.189 & 3.188 & 3.185 & 3.185 & 3.156 & 3.157 & 12.972 & 12.985 & 12.968 & .387 & .387 & .388 & .388 \\
\hline T3 & 50.767 & 15.409 & 3.179 & 3.179 & 3.180 & 3.180 & 3.155 & 3.154 & 12.886 & 12.879 & 12.889 & .389 & .389 & .387 & .387 \\
\hline T4 & 51.249 & 15.368 & 3.170 & 3.170 & 3.156 & 3.155 & 3.133 & 3.134 & 13.215 & 13.218 & 13.225 & .387 & .387 & .385 & .385 \\
\hline T5 & 51.241 & 15.361 & 3.160 & 3.160 & 3.167 & 3.167 & 3.090 & 3.090 & 13.247 & \begin{tabular}{|l}
13.239 \\
\end{tabular} & 13.228 & .386 & .386 & .385 & .386 \\
\hline T6 & 51.322 & 15.409 & 3.170 & 3.170 & 3.109 & 3.108 & 3.132 & 3.132 & 12.963 & 12.972 & 12.968 & .385 & .385 & .385 & .385 \\
\hline$T 7$ & 51.039 & 15.353 & 3.174 & 3.175 & 3.175 & 3.174 & 3.130 & 3.129 & 12.937 & 12.950 & 12.946 & .389 & .390 & .386 & .388 \\
\hline${ }^{*} \mathrm{~T} 10$ & 55.000 & 15.522 & 3.115 & 3.114 & 3.145 & 3.144 & 2.914 & 2.915 & 16.024 & \begin{tabular}{|l|}
16.024 \\
\end{tabular} & 16.034 & .308 & .309 & .326 & .327 \\
\hline "T11 & 57.751 & 15.656 & 3.028 & 3.027 & 3.047 & 3.047 & 2.749 & 2.749 & 18.194 & 18.206 & 18.198 & .289 & .290 & .301 & .301 \\
\hline${ }^{*} \mathrm{~T}$ 12 & 56.542 & 15.647 & 3.076 & 3.078 & 3.014 & 3.015 & 2.817 & 2.817 & 16.887 & 16.867 & 16.861 & .317 & .317 & .333 & .333 \\
\hline & & & & & & & & & & & & & & & \\
\hline & & & & & & & & & & & & & & & \\
\hline & & & & & & & & & & & & & & & \\
\hline & & & & & & & & & & & & & & & \\
\hline & & & & & & & & & & & & & & & \\
\hline & & & & & & & & & & & & & & & \\
\hline & & & & & & & & & & & & & & & \\
\hline
\end{tabular}

Measurement Notes (use back of sheet if needed):

Note: * Tensile specimens L10, L11, L12, L13, T10, T11, T12, were broke in such a way that it made it very difficult to line the specimens back up for inspection. The Conical indent marks were very faint and difficult to measure. 
Rev. 03

Title:

TEV No: :

Rev. No.:

2

Project No.:

25228

Date:

$05 / 02 / 18$

Foil $551-4$ Post Test Dimensions

Foil 551- 5 Post Test Dimensions Date: $7 / 23 / 14$ Tensile Specimen Dimensional Record Sheet (continuation)

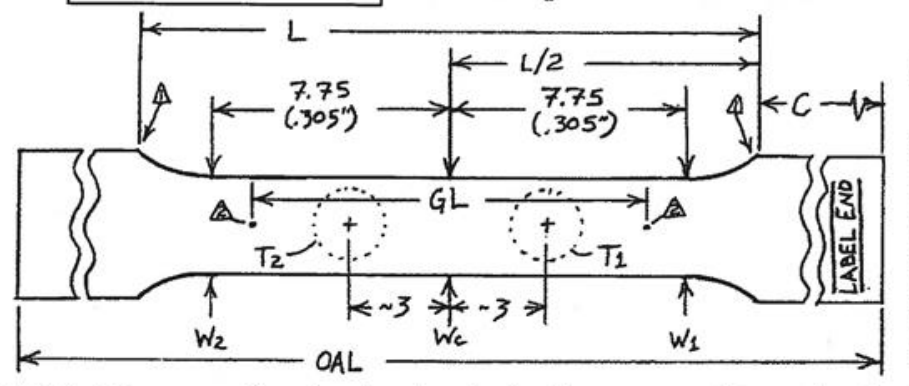
Measurement Operator Name: James Reseigh
Signature:
OAL measurement instrument ID: A678 Expiration Date: 4/9/15 GL measurement instrument ID: A678 Expiration Date: $4 / 9 / 15$ W measurement instrument ID: A678 Expiration Date: 4/9/15 T measurement instrument ID: A609 Temperature instrument ID: $A 52393 \quad$ Expiration Date: $6 / 23 / 15$ Expiration Date: $11 / 13 / 14$ Delta 1. Reference positions for reduced section locations - corner at intersection of end tab and fillet. Locate center between corners (L/2) as zero position reference. It is not necessary to record dimension $\mathrm{L}$. Delta 2. Conical indent marks for GL measurement are small, less than $0.1 \mathrm{~mm}$ typical. Measure indents center-to-center three times with optical comparator. Reset reference zero for each measurement (three independent measurements)

1. Locations to measure are indicated in the diagram. All dimensions in mm except as indicated by (in.). Record measurements to $0.001 \mathrm{~mm}$ excepting " $\mathrm{C}$ " and

2. Record dimension " $C$ " to $0.01 \mathrm{~mm}$ resolution.

3. Record dimension "OAL" to $0.01 \mathrm{~mm}$ resolution. This can be measured with a caliper.

4. Measure $W_{1}, W_{2}$ and $W_{c}$ two times. Reset width reference zero for each measurement (make two independent measurements at each location).

mark indent. Ball tip

6. Record room temperature before starting and after completion of each group of measurements.

\begin{tabular}{|c|c|c|c|c|c|c|c|c|c|c|c|c|c|c|c|}
\hline \multirow{2}{*}{$\begin{array}{c}\text { Specimen } \\
\text { ID }\end{array}$} & \multirow{2}{*}{$\begin{array}{l}\mathrm{OAL} \\
(\mathrm{mm})\end{array}$} & \multirow{2}{*}{$\begin{array}{c}\mathrm{C} \\
(\mathrm{mm})\end{array}$} & \multicolumn{2}{|c|}{$\mathrm{W}_{1}(\mathrm{~mm})$} & \multicolumn{2}{|c|}{$\mathrm{W}_{2}(\mathrm{~mm})$} & \multicolumn{2}{|c|}{$W_{c}(\mathrm{~mm})$} & \multicolumn{3}{|c|}{$\overline{\mathrm{GL}}(\mathrm{mm})$} & \multicolumn{2}{|c|}{$T_{1}(\mathrm{~mm})$} & \multicolumn{2}{|c|}{$\mathrm{T}_{2}(\mathrm{~mm})$} \\
\hline & & & $\# 1$ & $\# 2$ & $\# 1$ & $\# 2$ & $\# 1$ & $\# 2$ & $\# 1$ & $\# 2$ & $\# 3$ & $\# 1$ & $\# 2$ & $\# 1$ & $\# 2$ \\
\hline $551-5 \mathrm{~L} 7$ & 52.039 & 15.436 & 3.156 & 3.155 & 3.157 & 3.156 & 3.099 & 3.100 & 13.819 & 13.824 & 13.820 & .526 & .526 & .528 & .528 \\
\hline L8 & 52.116 & 15.468 & 3.157 & 3.157 & 3.138 & 3.140 & 3.102 & 3.103 & 13.775 & 13.773 & 13.778 & .527 & .527 & .525 & .525 \\
\hline L9 & 52.382 & 15.436 & 3.141 & 3.142 & 3.135 & 3.136 & 3.092 & 3.092 & 14.119 & 14.118 & 14.108 & .525 & .525 & .523 & .523 \\
\hline $551-5$ T13 & 51.936 & 15.393 & 3.159 & 3.158 & 3.151 & 3.151 & 3.122 & 3.122 & 13.764 & 13.748 & 13.758 & .534 & .534 & .535 & .535 \\
\hline $551-4$ L7 & 50.634 & 15.350 & 3.181 & 3.180 & 3.183 & 3.183 & 3.112 & 3.112 & 13.051 & 13.063 & 13.060 & .393 & .393 & .394 & .394 \\
\hline L8 & 50.575 & 15.398 & 3.207 & 3.206 & 3.190 & 3.190 & 3.178 & 3.178 & 13.053 & 13.045 & 13.049 & .391 & .391 & .394 & .394 \\
\hline "L9 & 50.549 & 15.412 & 3.187 & 3.187 & 3.187 & 3.188 & 3.168 & 3.166 & 12.806 & 12.790 & 12.803 & .389 & .389 & .391 & .391 \\
\hline & & & & & & & & & & & & & & & \\
\hline
\end{tabular}

Measurement Notes (use back of sheet if needed): * 551-4-L.9 Specimen broke at Conical indent mark. GL measurement difficult to align on indent mark. 
Rev. 03

Title:

TEV No.: 2194

Rev. No.:

2

Project No.:

25228

Date:

$05 / 02 / 18$

Foil 551-5 PreTest Dimensions

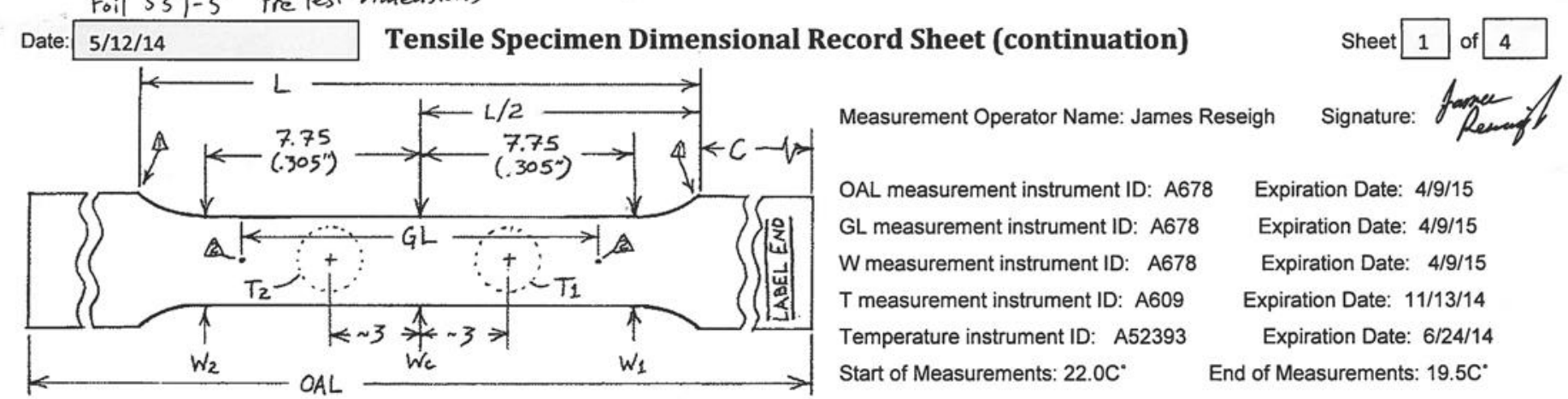

Delta 1. Reference positions for reduced section locations - corner at intersection of end tab and fillet. Locate center between corners (L/2) as zero position reference.

It is not necessary to record dimension $\mathrm{L}$.
Delta 2 . Conical indent marks for $\mathrm{GL}$ measurement are small, less than $0.1 \mathrm{~mm}$ typical. Measure indents center-to-center three times with optical comparator. Reset reference zero for each measurement (three independent measurements).

1. Locations to measure are indicated in the diagram. All dimensions in mm except as indicated by (in.). Record measurements to $0.001 \mathrm{~mm}$ excepting "C" and

2. Record dimension "C" to $0.01 \mathrm{~mm}$ resolution.

3. Record dimension "OAL" to $0.01 \mathrm{~mm}$ resolution. This can be measured with a caliper.

4. Measure $W_{1}, W_{2}$, and $W_{c}$ two times. Reset width reference zero for each measurement (make two independent measurements at each location).

. Measure thicknesses $T_{1}$ and $T_{2}$, twice at each location, approximately $3 \mathrm{~mm}$ either side of centerline. Measuring device contact surface should not cover the gage mark indent. Ball tip micrometer use is suggested for small contact area on indent face of specimen. Use minimum pressure needed to achieve consistent measurements.

6. Record room temperature before starting and after completion of each group of measurements.

\begin{tabular}{|c|c|c|c|c|c|c|c|c|c|c|c|c|c|c|c|}
\hline \multirow{2}{*}{$\begin{array}{c}\text { Specimen } \\
\text { ID }\end{array}$} & \multirow{2}{*}{$\begin{array}{l}\text { OAL } \\
(\mathrm{mm})\end{array}$} & \multirow{2}{*}{$\begin{array}{c}\mathrm{C} \\
(\mathrm{mm})\end{array}$} & \multicolumn{2}{|c|}{$\mathrm{W}_{1}(\mathrm{~mm})$} & \multicolumn{2}{|c|}{$\mathrm{W}_{2}(\mathrm{~mm})$} & \multicolumn{2}{|c|}{$\mathrm{W}_{\mathrm{c}}(\mathrm{mm})$} & \multicolumn{3}{|c|}{$\mathrm{GL}(\mathrm{mm})$} & \multicolumn{2}{|c|}{$T_{1}(\mathrm{~mm})$} & \multicolumn{2}{|c|}{$\mathrm{T}_{2}(\mathrm{~mm})$} \\
\hline & & & $\# 1$ & $\# 2$ & $\# 1$ & \#2 & $\# 1$ & \#2 & $\# 1$ & $\# 2$ & $\# 3$ & $\# 1$ & \#2 & $\# 1$ & $\# 2$ \\
\hline $\mathrm{L} 1$ & 50.710 & 15.361 & 3.192 & 3.192 & 3.196 & 3.196 & 3.161 & 3.163 & 12.726 & 12.735 & 12.728 & .523 & .523 & .521 & .521 \\
\hline L2 & 50.707 & 15.369 & 3.206 & 3.206 & 3.199 & 3.199 & 3.174 & 3.174 & 12.721 & 12.710 & 12.714 & .533 & .533 & .532 & .532 \\
\hline L3 & 50.701 & 15.377 & 3.191 & 3.191 & 3.196 & 3.196 & 3.168 & 3.168 & 12.699 & 12.693 & 12.695 & .540 & .540 & .535 & .535 \\
\hline L4 & 50.695 & 15.374 & 3.192 & 3.193 & 3.204 & 3.203 & 3.164 & 3.165 & 12.835 & 12.857 & 12.856 & .532 & .532 & .535 & .536 \\
\hline L5 & 50.694 & 15.395 & 3.193 & 3.193 & 3.195 & 3.195 & 3.175 & 3.175 & 12.688 & 12.691 & 12.692 & .506 & .506 & .516 & .516 \\
\hline L6 & 50.700 & 15.365 & 3.189 & 3.189 & 3.208 & 3.208 & 3.175 & 3.174 & 12.771 & 12.771 & 12.766 & .520 & .520 & .520 & .521 \\
\hline L7 & 50.699 & 15.385 & 3.194 & 3.194 & 3.202 & 3.202 & 3.176 & 3.176 & 12.726 & 12.729 & 12.731 & .544 & .544 & .541 & .541 \\
\hline L8 & 50.706 & 15.407 & 3.202 & 3.202 & 3.191 & 3.192 & 3.179 & 3.179 & 12.681 & 12.673 & 12.672 & .541 & .542 & .538 & .538 \\
\hline
\end{tabular}


TEM-10300-1

03/01/2012

TECHNICAL EVALUATION

Page B33 of B35

Rev. 03

Title:

Elevated Temperature Tensile Tests on DU-10Mo Rolled Foils

TEV No.: 2194

Rev. No.:

2

Project No.:

25228

Date:

05/02/18

Date: $5 / 12 / 14$

Tensile Specimen Dimensional Record Sheet (continuation)

Sheet 2 of 4

Measurement Operator Name:

signature: farme thengyt

\begin{tabular}{|c|c|c|c|c|c|c|c|c|c|c|c|c|c|c|c|}
\hline \multirow{2}{*}{$\begin{array}{l}\text { Specimen } \\
\text { ID }\end{array}$} & \multirow{2}{*}{$\begin{array}{l}\mathrm{OAL} \\
(\mathrm{mm}) \\
\end{array}$} & \multirow{2}{*}{$\frac{C}{(\mathrm{~mm})}$} & \multicolumn{2}{|c|}{$\mathrm{W}_{1}(\mathrm{~mm})$} & \multicolumn{2}{|c|}{$\mathrm{W}_{2}(\mathrm{~mm})$} & \multicolumn{2}{|c|}{$W_{c}(\mathrm{~mm})$} & \multicolumn{3}{|c|}{$\mathrm{GL}(\mathrm{mm})$} & \multicolumn{2}{|c|}{$T_{1}(\mathrm{~mm})$} & \multicolumn{2}{|c|}{$T_{2}(\mathrm{~mm})$} \\
\hline & & & $\# 1$ & $\# 2$ & $\# 1$ & $\# 2$ & $\# 1$ & \#2 & $\# 1$ & $\# 2$ & $\# 3$ & $\# 1$ & \#2 & $\# 1$ & $\# 2$ \\
\hline L9 & 50.709 & 15.389 & 3.201 & 3.201 & 3.195 & 3.197 & 3.169 & 3.169 & 12.767 & 12.765 & 12.761 & .542 & .542 & .540 & .541 \\
\hline L10 & 50.709 & 15.389 & 3.198 & 3.198 & 3.195 & 3.196 & 3.180 & 3.180 & 12.783 & 12.787 & 12.788 & .536 & .536 & .535 & .535 \\
\hline L11 & 50.708 & 15.406 & 3.189 & 3.189 & 3.187 & 3.187 & 3.166 & 3.165 & 12.642 & 12.636 & 12.632 & .533 & .533 & .534 & .534 \\
\hline L12 & 50.711 & 15.384 & 3.197 & 3.197 & 3.198 & 3.198 & 3.170 & 3.170 & 12.744 & 12.730 & 12.729 & .527 & .527 & .525 & .525 \\
\hline L13 & 50.710 & 15.406 & 3.195 & 3.196 & 3.187 & 3.187 & 3.173 & 3.172 & 12.752 & 12.748 & 12.740 & .519 & .519 & .518 & .518 \\
\hline L14 & 50.716 & 15.384 & 3.194 & 3.194 & 3.198 & 3.199 & 3.165 & 3.163 & 12.744 & 12.748 & 12.753 & .505 & $.50+6$ & .504 & .503 \\
\hline$\angle 29$ & 50.703 & 15.129 & 3.190 & 3.190 & 3.190 & 3.190 & 3.179 & 3.179 & 12.720 & 12.713 & 12.708 & .530 & .530 & .530 & .530 \\
\hline L30 & 50.704 & 15.172 & 3.195 & 3.194 & 3.201 & 3.201 & 3.179 & 3.180 & 12.768 & 12.770 & 12.769 & .541 & .541 & .547 & .547 \\
\hline L31 & 50.704 & 15.189 & 3.205 & 3.205 & 3.213 & 3.213 & 3.191 & 3.191 & 12.775 & 12.766 & 12.770 & .550 & .550 & .550 & .550 \\
\hline L32 & 50.699 & 15.207 & 3.201 & 3.201 & 3.215 & 3.214 & 3.189 & 3.189 & 12.732 & 12.734 & 12.733 & .550 & .550 & .554 & .554 \\
\hline L33 & 50.698 & 15.207 & 3.199 & 3.199 & 3.207 & 3.207 & 3.186 & 3.186 & 12.713 & 12.716 & 12.720 & .521 & .521 & .526 & .526 \\
\hline L34 & 50.699 & 15.244 & 3.198 & 3.197 & 3.217 & 3.217 & 3.187 & 3.187 & 12.715 & 12.720 & 12.718 & .550 & .551 & .555 & .555 \\
\hline L35 & 50.708 & 15.250 & 3.193 & 3.193 & 3.202 & 3.202 & 3.186 & 3.185 & 12.780 & 12.774 & 12.777 & .552 & .552 & .555 & .555 \\
\hline L36 & 50.697 & 15.275 & 3.203 & 3.203 & 3.206 & 3.207 & 3.183 & 3.184 & 12.762 & 12.769 & 12.776 & .554 & .554 & .554 & .554 \\
\hline L37 & 50.700 & 15.288 & 3.203 & 3.203 & 3.201 & 3.201 & 3.182 & 3.182 & 12.737 & 12.739 & 12.742 & .546 & .546 & .546 & .546 \\
\hline L38 & 50.697 & 15.322 & 3.200 & 3.200 & 3.222 & 3.221 & 3.193 & 3.193 & 12.761 & 12.758 & 12.761 & .548 & .548 & .549 & .549 \\
\hline L39 & 50.698 & 15.324 & 3.204 & 3.204 & 3.213 & 3.213 & 3.191 & 3.191 & 12.733 & 12.725 & 12.730 & .542 & .542 & .545 & .545 \\
\hline L40 & 50.696 & 15.346 & 3.198 & 3.198 & 3.218 & 3.218 & 3.192 & 3.192 & 12.691 & 12.703 & 12.702 & .541 & .541 & .541 & .542 \\
\hline L41 & 50.700 & 15.345 & 3.199 & 3.199 & 3.202 & 3.202 & 3.198 & 3.198 & 12.713 & 12.715 & 12.716 & .524 & .524 & .530 & .530 \\
\hline
\end{tabular}

Measurement Notes (use back of sheet if needed): L29 specimen is not full width 
TEM-10300-1

03/01/2012

TECHNICAL EVALUATION

Page B34 of B35

Rev. 03

Title:

Elevated Temperature Tensile Tests on DU-10Mo Rolled Foils

TEV No.: 2194

Rev. No.:

2

Project No.:

25228

Date:

05/02/18

Date: $5 / 12 / 14$

Tensile Specimen Dimensional Record Sheet (continuation)

Sheet 3 of 4

Measurement Operator Name:

signature: farme therngt

\begin{tabular}{|c|c|c|c|c|c|c|c|c|c|c|c|c|c|c|c|}
\hline \multirow{2}{*}{$\begin{array}{c}\text { Specimen } \\
\text { ID }\end{array}$} & \multirow{2}{*}{$\begin{array}{l}\text { OAL } \\
(\mathrm{mm})\end{array}$} & \multirow{2}{*}{$\begin{array}{c}\mathrm{C} \\
(\mathrm{mm})\end{array}$} & \multicolumn{2}{|c|}{$W_{1}(\mathrm{~mm})$} & \multicolumn{2}{|c|}{$W_{2}(\mathrm{~mm})$} & \multicolumn{2}{|c|}{$W_{c}(\mathrm{~mm})$} & \multicolumn{3}{|c|}{$\mathrm{GL}(\mathrm{mm})$} & \multicolumn{2}{|c|}{$T_{1}(\mathrm{~mm})$} & \multicolumn{2}{|c|}{$\mathrm{T}_{2}(\mathrm{~mm})$} \\
\hline & & & $\# 1$ & \#2 & $\# 1$ & \#2 & $\# 1$ & \#2 & $\# 1$ & \#2 & $\# 3$ & $\# 1$ & $\# 2$ & $\# 1$ & $\# 2$ \\
\hline L42 & 50.698 & 15.363 & 3.194 & 3.193 & 3.197 & 3.197 & 3.184 & 3.184 & 12.754 & 12.750 & 12.751 & .531 & .531 & .531 & .532 \\
\hline T1 & 50.755 & 15.338 & 3.218 & 3.217 & 3.221 & 3.222 & 3.192 & 3.192 & 12.744 & 12.748 & 12.744 & .531 & .531 & .540 & .540 \\
\hline T2 & 50.764 & 15.341 & 3.174 & 3.174 & 3.173 & 3.174 & 3.145 & 3.146 & 12.731 & 12.720 & 12.724 & .543 & .543 & .539 & .539 \\
\hline T3 & 50.772 & 15.384 & 3.202 & 3.203 & 3.191 & 3.191 & 3.174 & 3.175 & 12.684 & 12.691 & 12.682 & .541 & .541 & .540 & .540 \\
\hline T4 & 50.785 & 15.335 & 3.195 & 3.195 & 3.192 & 3.191 & 3.173 & 3.174 & 12.693 & 12.690 & 12.689 & .540 & .539 & .540 & .540 \\
\hline T5 & 50.783 & 15.382 & 3.196 & 3.196 & 3.198 & 3.198 & 3.168 & 3.167 & 12.747 & 12.739 & 12.746 & .539 & .539 & .531 & .531 \\
\hline T6 & 50.784 & 15.362 & 3.213 & 3.212 & 3.195 & 3.195 & 3.175 & 3.175 & 12.749 & 12.744 & 12.747 & .536 & .536 & .536 & .536 \\
\hline $\mathrm{T7}$ & 50.788 & 15.385 & 3.196 & 3.196 & 3.201 & 3.201 & 3.172 & 3.172 & 12.672 & 12.671 & 12.664 & .538 & .538 & .539 & .539 \\
\hline T8 & 50.804 & 15.386 & 3.200 & 3.201 & 3.192 & 3.192 & 3.172 & 3.172 & 12.705 & 12.707 & 12.710 & .538 & .538 & .540 & .539 \\
\hline T9 & 50.784 & 15.390 & 3.201 & 3.201 & 3.200 & 3.198 & 3.172 & 3.173 & 12.807 & 12.808 & 12.803 & .540 & .540 & .539 & .539 \\
\hline T10 & 50.787 & 15.394 & 3.195 & 3.194 & 3.194 & 3.194 & 3.171 & 3.171 & 12.736 & 12.743 & 12.734 & .539 & .539 & .539 & .539 \\
\hline T11 & 50.784 & 15.379 & 3.197 & 3.198 & 3.197 & 3.197 & 3.177 & 3.177 & 12.748 & 12.758 & 12.758 & .538 & .538 & .538 & .538 \\
\hline T12 & 50.809 & 15.383 & 3.203 & 3.203 & 3.195 & 3.196 & 3.173 & 3.173 & 12.678 & 12.678 & 12.680 & .541 & .541 & .539 & .539 \\
\hline T13 & 50.779 & 15.406 & 3.193 & 3.194 & 3.194 & 3.194 & 3.176 & 3.177 & 12.746 & 12.738 & 12.738 & .541 & .541 & .540 & .540 \\
\hline T14 & 50.781 & 15.397 & 3.211 & 3.211 & 3.191 & 3.192 & 3.181 & 3.181 & 12.693 & 12.691 & 12.691 & .539 & .539 & .537 & .537 \\
\hline T15 & 50.766 & 15.384 & 3.187 & 3.186 & 3.203 & 3.203 & 3.165 & 3.164 & 12.695 & 12.705 & 12.708 & .543 & .543 & .538 & .538 \\
\hline T16 & 50.748 & 15.395 & 3.210 & 3.211 & 3.193 & 3.193 & 3.172 & 3.172 & 12.751 & 12.755 & 12.746 & .538 & .539 & .540 & .540 \\
\hline T17 & 50.740 & 15.397 & 3.191 & 3.191 & 3.202 & 3.202 & 3.175 & 3.175 & 12.720 & 12.726 & 12.724 & .546 & .546 & .543 & .543 \\
\hline T18 & 50.729 & 15.403 & 3.213 & 3.214 & 3.195 & 3.196 & 3.171 & 3.171 & 12.672 & 12.664 & 12.662 & .529 & .530 & .539 & .539 \\
\hline
\end{tabular}

Measurement Notes (use back of sheet if needed): 
Rev. 03

Elevated Temperature Tensile Tests on DU-10Mo Rolled Foils

TEV No.:

Rev. No.:

2

Project No: :

25228

Date:

$05 / 02 / 18$

Date: $5 / 12 / 14$ Measurement Operator Name:

\section{Tensile Specimen Dimensional Record Sheet (continuation)}

Signatur
Sheet 4 of 4

\begin{tabular}{|c|c|c|c|c|c|c|c|c|c|c|c|c|c|c|c|}
\hline \multirow{2}{*}{$\begin{array}{c}\text { Specimen } \\
\text { ID }\end{array}$} & \multirow{2}{*}{$\begin{array}{l}\mathrm{OAL} \\
(\mathrm{mm})\end{array}$} & \multirow{2}{*}{$\begin{array}{c}\mathrm{C} \\
(\mathrm{mm})\end{array}$} & \multicolumn{2}{|c|}{$\mathrm{W}_{1}(\mathrm{~mm})$} & \multicolumn{2}{|c|}{$\mathrm{W}_{2}(\mathrm{~mm})$} & \multicolumn{2}{|c|}{$\mathrm{W}_{\mathrm{c}}(\mathrm{mm})$} & \multicolumn{3}{|c|}{$\mathrm{GL}(\mathrm{mm})$} & \multicolumn{2}{|c|}{$T_{1}(\mathrm{~mm})$} & \multicolumn{2}{|c|}{$T_{2}(\mathrm{~mm})$} \\
\hline & & & $\# 1$ & \#2 & $\# 1$ & \#2 & $\# 1$ & \#2 & $\# 1$ & $\# 2$ & $\# 3$ & $\# 1$ & \#2 & $\# 1$ & $\# 2$ \\
\hline T19 & 50.969 & 15.421 & 3.198 & 3.198 & 3.203 & 3.204 & 3.163 & 3.162 & 12.800 & 12.804 & 12.809 & .534 & .534 & .538 & .538 \\
\hline & & & & & & & & & & & & & & & \\
\hline & & & & & & & & & & & & & & & \\
\hline & & & & & & & & & & & & & & & \\
\hline & & & & & & & & & & & & & & & \\
\hline & & & & & & & & & & & & & & & \\
\hline & & & & & & & & & & & & & & & \\
\hline & & & & & & & & & & & & & & & \\
\hline & & & & & & & & & & & & & & & \\
\hline & & & & & & & & & & & & & & & \\
\hline & & & & & & & & & & & & & & & \\
\hline & & & & & & & & & & & & & & & \\
\hline & & & & & & & & & & & & & & & \\
\hline & & & & & & & & & & & & & & & \\
\hline & & & & & & & & & & & & & & & \\
\hline & & & & & & & & & & & & & & & \\
\hline & & & & & & & & & & & & & & & \\
\hline & & & & & & & & & & & & & & & \\
\hline & & & & & & & & & & & & & & & \\
\hline & & & & & & & & & & & & & & & \\
\hline
\end{tabular}

Measurement Notes (use back of sheet if needed): 